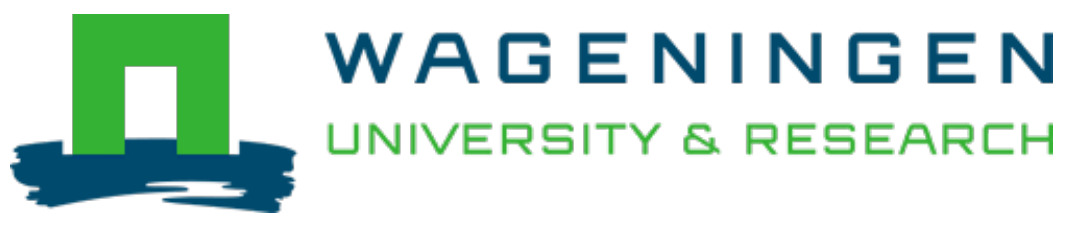

\title{
Rethinking organic wastes bioconversion: Evaluating the potential of the black soldier fly (Hermetia illucens (L.)) (Diptera: Stratiomyidae) (BSF)
}

\section{Waste Management}

Surendra, K.C.; Tomberlin, Jeffery K.; Huis, Arnold; Cammack, Jonathan A.; Heckmann, Lars Henrik L. et al https://doi.org/10.1016/j.wasman.2020.07.050

This publication is made publicly available in the institutional repository of Wageningen University and Research, under the terms of article $25 \mathrm{fa}$ of the Dutch Copyright Act, also known as the Amendment Taverne. This has been done with explicit consent by the author.

Article 25 fa states that the author of a short scientific work funded either wholly or partially by Dutch public funds is entitled to make that work publicly available for no consideration following a reasonable period of time after the work was first published, provided that clear reference is made to the source of the first publication of the work.

This publication is distributed under The Association of Universities in the Netherlands (VSNU) 'Article $25 \mathrm{fa}$ implementation' project. In this project research outputs of researchers employed by Dutch Universities that comply with the legal requirements of Article $25 \mathrm{fa}$ of the Dutch Copyright Act are distributed online and free of cost or other barriers in institutional repositories. Research outputs are distributed six months after their first online publication in the original published version and with proper attribution to the source of the original publication.

You are permitted to download and use the publication for personal purposes. All rights remain with the author(s) and / or copyright owner(s) of this work. Any use of the publication or parts of it other than authorised under article $25 \mathrm{fa}$ of the Dutch Copyright act is prohibited. Wageningen University \& Research and the author(s) of this publication shall not be held responsible or liable for any damages resulting from your (re)use of this publication.

For questions regarding the public availability of this publication please contact openscience.library@wur.nl 


\section{Rethinking organic wastes bioconversion: Evaluating the potential of the black soldier fly (Hermetia illucens (L.)) (Diptera: Stratiomyidae) (BSF)}

\section{K.C. Surendra ${ }^{\mathrm{a}}$, Jeffery K. Tomberlin ${ }^{\mathrm{b}}$, Arnold van Huis ${ }^{\mathrm{c}}$, Jonathan A. Cammack ${ }^{\mathrm{b}}$, Lars-Henrik L. Heckmann ${ }^{\mathrm{d}}$, Samir Kumar Khanal ${ }^{\text {a,* }}$}

a Department of Molecular Biosciences and Bioengineering, University of Hawai'i at Mānoa, Honolulu, HI 96822, USA

${ }^{\mathrm{b}}$ Department of Entomology, Texas AE'M University, College Station, TX, 77843, USA

' Laboratory of Entomology, Wageningen University \&' Research, Wageningen, the Netherlands

d Danish Technological Institute, Life Science, Kongsvang Allé 29, 8000 Aarhus C, Denmark

\section{A R T I C L E I N F O}

\section{Article history:}

Received 1 May 2020

Revised 15 July 2020

Accepted 24 July 2020

\section{Keywords:}

Organic wastes

Bioconversion

Biorefinery

Black soldier fly

Feed

Biodiesel

Organic fertilizer

Biobased products

Bioactive compounds

\begin{abstract}
A B S T R A C T
Population growth and unprecedented economic growth and urbanization, especially in low- and middle-income countries, coupled with extreme weather patterns, the high-environmental footprint of agricultural practices, and disposal-oriented waste management practices, require significant changes in the ways we produce food, feed and fuel, and manage enormous amounts of organic wastes. Farming insects such as the black soldier fly (BSF) (Hermetia illucens) on diverse organic wastes provides an opportunity for producing nutrient-rich animal feed, fuel, organic fertilizer, and biobased products with concurrent valorization of wastes. Inclusion of BSF larvae/pupae in the diets of poultry, fish, and swine has shown promise as a potential substitute of conventional feed ingredients such as soybean meal and fish meal. Moreover, the bioactive compounds such as antimicrobial peptides, medium chain fatty acids, and chitin and its derivatives present in BSF larvae/pupae, could also add values to the animal diets. However, to realize the full potential of BSF-based biorefining, more research and development efforts are necessary for scaling up the production and processing of BSF biomass using more mechanized and automated systems. More studies are also needed to ensure the safety of the BSF biomass grown on various organic wastes for animal feed (also food) and legalizing the feed application of BSF biomass to wider categories of animals. This critical review presents the current status of the BSF technology, identifies the research gaps, highlights the challenges towards industrial scale production, and provides future perspectives.
\end{abstract}

() 2020 Elsevier Ltd. All rights reserved.

\section{Contents}

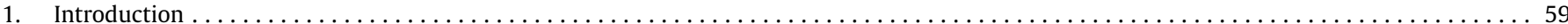

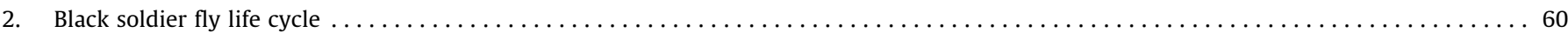

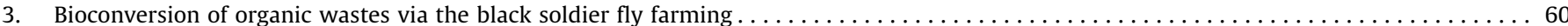

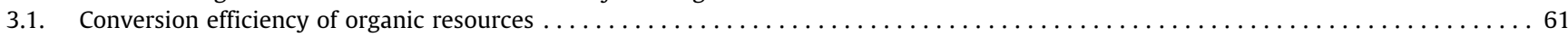

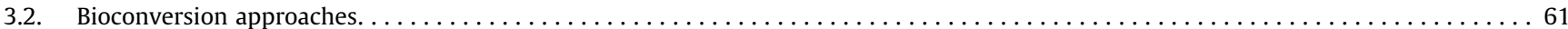

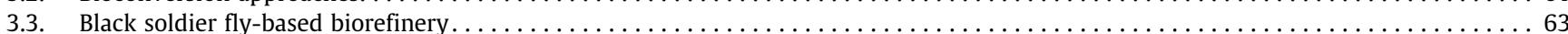

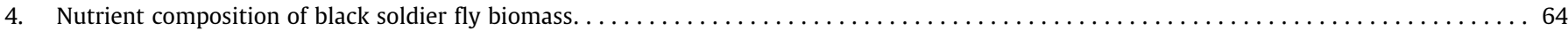

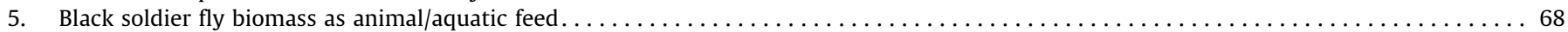

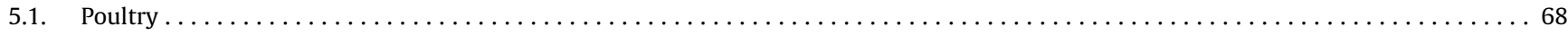

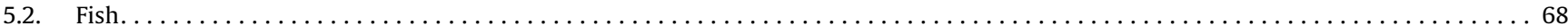

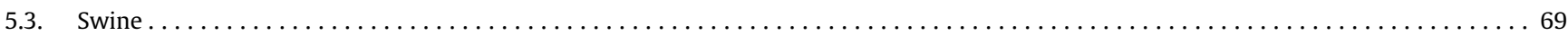

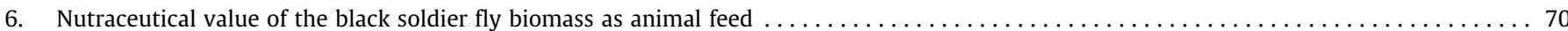

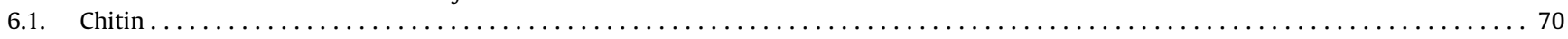

\footnotetext{
* Corresponding author.

E-mail address: khanal@hawaii.edu (S.K. Khanal).
} 


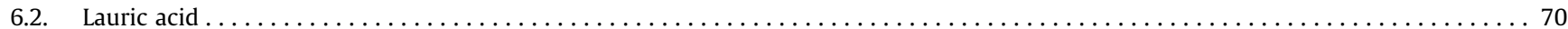

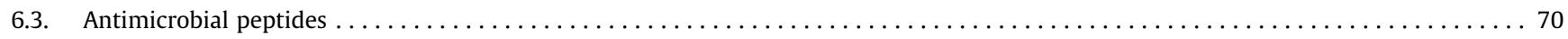

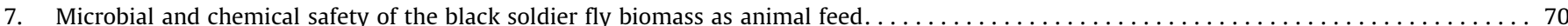

8. Environmental aspects of bioconversion of organic wastes into black soldier fly biomass . . . . . . . . . . . . . . . . . . . . 71

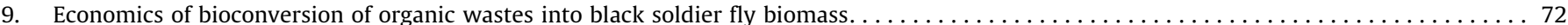

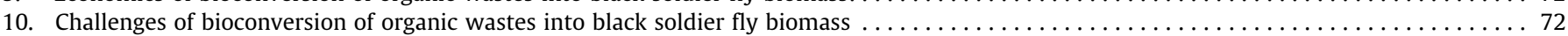

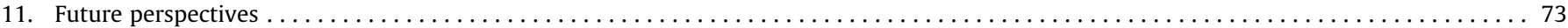

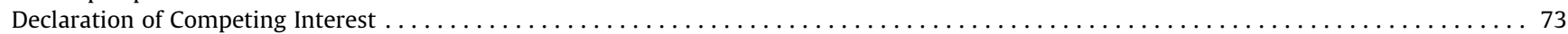

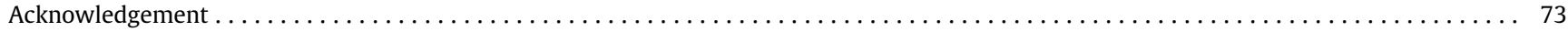

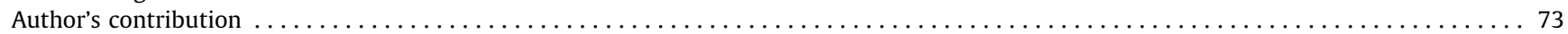

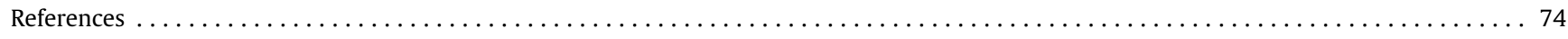

\section{Introduction}

With the rapid population growth, economic development, and urbanization, especially in low- and middle-income countries, meeting the demand for quality food and managing organic waste will require substantial resources. For example, the rapid increase in per capita income and urbanization is likely to result in structural changes in demand for food and other agricultural products (FAO, 2017; Surendra and Kuehnle, 2019). To meet the demand for these resources, agricultural production is projected to increase by $49 \%$ between 2013 and 2050 (FAO, 2017), while, from $2005 / 2007$ to 2050 , meat, egg, and dairy production are projected to increase by $76 \%, 64 \%$, and $62 \%$, respectively (Alexandratos and Bruinsma, 2012). Similarly, global food fish production in 2030 is projected to be $17.6 \%$ higher ( 30 million metric tons live weight equivalent) than that in 2016 (FAO, 2018). Since about six units of plant protein are required to produce one unit of high-quality animal protein (Pimentel and Pimentel, 2003), the projected increase in meat and dairy production will result in substantial increase in demand for protein-rich animal feeds (Surendra and Kuehnle, 2019). A significant amount of organic waste is generated worldwide and is not properly managed, especially in low- and middle-income countries (Kaza et al., 2018; Troschinetz and Mihelcic, 2009; Wilson et al., 2012). Annually, a third of all food produced (about 1.7 billion metric tons) is lost or wasted along the food chain, which has a significant environmental (3.3 billion metric tons of $\mathrm{CO}_{2}$-equivalent greenhouse gas (GHG) emission per year) and economic (total social costs of $\$ 1.2$ trillion per year) footprints (FAO, 2014). Moreover, high moisture and nutrient contents, biological instability due to biodegradation and enzymatic activity, and the potential presence of pathogens make the organic waste management more challenging in comparison to other waste streams (Russ and Meyer-Pittroff, 2004; Varelas, 2019; Yin et al., 2014). Currently, landfilling and haphazard land disposal dominate the waste disposal methods, especially in low- and middle-income countries, resulting in substantial environmental (ground- and surface-water pollution and GHG emissions) (Bogner et al., 2008; Reddy and Nandini, 2011), public health (breeding ground for disease vectors) (Lohri et al., 2017; Reddy and Nandini, 2011), economic (transportation cost), and social (not-in-my-backyard) issues.

Overall, environmental, economic, public health, and food security issues associated with the ever-increasing and non-sustainable way of using limited resources for food and feed production, coupled with the generation of large amounts of organic wastes, requires a more economically-viable, environmentally-friendly, and technically-feasible approach to food and feed production and organic waste management. In this context, insect farming on organic wastes provides an avenue for wastes bioremediation with concurrent generation of nutrient-rich feed (i.e., insect biomass) and organic fertilizer (Surendra and Kuehnle, 2019). Farming insects on different organic wastes such as food waste, human feces, and animal manure has been reported to reduce the organic waste by $25-72 \%$ (dry matter basis) (Diener et al., 2011; Henry et al., 2015; Lalander et al., 2015, 2019, Nyakeri et al., 2017b, 2019, Rehman et al., 2017a, 2017b, 2019; Somroo et al., 2019; Xiao et al., 2018b; Zhou et al., 2013) and nutrients such as nitrogen and phosphorus by $22-57 \%$ and $35-70 \%$, respectively (Lalander et al., 2015; Myers et al., 2008; Rehman et al., 2017; Zhou et al., 2013). Many insects belonging to the orders Lepidoptera, Diptera, Hymenoptera, Coleoptera, Trichoptera, Hemiptera, and Odonata exhibit antifungal activity and/or antimicrobial peptides (Elhag et al., 2017; Henry et al., 2015); hence, insect farming on organic wastes significantly reduces pathogens (Erickson et al., 2004; Henry et al., 2015; Lalander et al., 2015; Liu et al., 2008) and odors (Beskin et al., 2018).

Several insects, such as mealworms (Tenebrio molitor, Alphitobius diaperinus, and Zophobas morio), locusts (e.g., Locusta migratoria and Schistocerca gregaria), crickets (e.g., Acheta domestica and Gryllodes sigillatus), house fly (Musca domestica), and the black soldier fly (Hermetia illucens) (BSF) have been identified as key players. However, the BSF, which is a detritivorous insect has received significant attention due to its ability to grow on diverse organic wastes, such as livestock manure (Beskin et al., 2018; Chen et al., 2019; Julita et al., 2018; Lalander et al., 2015; Li et al., 2011; Liu et al., 2008; Mazza et al., 2020; Moula et al., 2018; Myers et al., 2008; Newton et al., 2005; Oonincx et al., 2015; Rehman et al., 2017; Sheppard, 1983; Sheppard et al., 1994; Xiao et al., 2018b; Yu et al., 2011; Zhou et al., 2013), human feces (Banks et al., 2014; Lalander et al., 2013, 2015; Nyakeri et al., 2017b), organic fraction of municipal solid waste (Cai et al., 2018; Diener et al., 2011; Sarpong et al., 2019), food wastes (Nguyen et al., 2015; Nyakeri et al., 2017b; Surendra et al., 2016; Zheng et al., 2012), agri-residues (Lim et al., 2019; Mohd-Noor et al., 2017; Palma et al., 2018; Supriyatna et al., 2016), compost leachate (Green and Popa, 2012; Popa and Green, 2012), landfill leachate (Grossule and Lavagnolo, 2019), insect farm waste (Jucker et al., 2020), fish offal (St-Hilaire et al., 2007a), and vertebrate remains (decomposing swine carcasses) (Tomberlin et al., 2005); thereby producing protein- and fat-rich larval, prepupal, or pupal biomass (referred to as BSF biomass hereafter unless stated otherwise) for animal feed and biofuel applications. Moreover, the BSF is not known to vector pathogens or to be a nuisance to companion animals or humans (Diener, 2010; Furman et al., 1959).

The BSF has been studied for organic waste valorization and animal feed production for over seven decades. However, the potential of BSF technology for bioconversion of organic wastes into value-added products has not been well examined. There are 


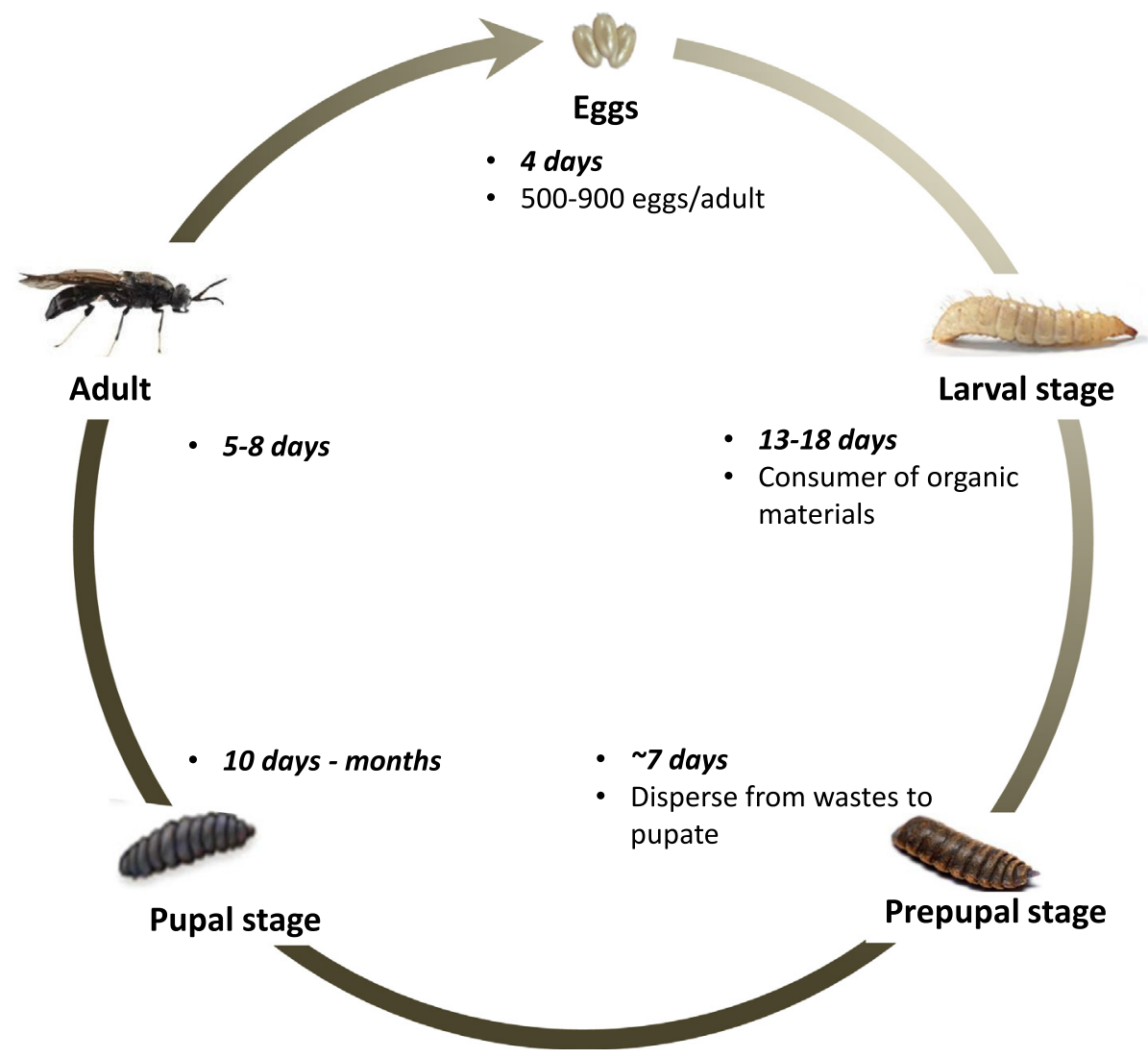

Fig. 1. A schematic of life cycle of the BSF.

only a few commercial-scale organic waste bioconversion facilities using the BSF, and these are sparsely distributed globally. Thus, the overarching goal of this review is to critically evaluate the current state-of-the-art of bioconversion of organic wastes via BSF farming with emphasis on conversion efficiency, biomass yield, factors affecting bioconversion, nutrient composition, and BSF biomass as an animal feed. This review also highlights the technical challenge associated with insect farming on organic wastes for animal feed applications. The environmental and economic aspects of bioconversion of organic wastes into BSF biomass are also briefly discussed. The review concludes with recommendations for further research and development, and commercialization of the technology.

\section{Black soldier fly life cycle}

The BSF has a historic distribution throughout the Nearctic region of the world. However, with globalization and technological advancements, this species can now be found in most temperate and tropic regions (Oliveira et al., 2015; Roháček and Hora, 2013; Sheppard et al., 1994). The BSF is wasp-like in appearance with unique color patterns of white and black over its body (Tomberlin et al., 2002). However, the BSF may also appear like other species of Hermetia and can easily be confused; adult specimens require verification by a taxonomic expert prior to commercialization. The life cycle of the BSF (Fig. 1) is like other holometabolous arthropods (May 1961). Adults live for approximately two weeks when only provided water. Usually males emerge two days prior to females and mating occurs two days later. The BSF is an eurygamous (mating on flight) insect, and thus requires broad areas for its nuptial flight (Caruso et al., 2013). They typically mate once, and females will lay one clutch of eggs during their life, usually two days after mating. Resulting eggs hatch in about four days with larvae needing about two weeks to complete development (Tomberlin et al., 2002). The prepupae, the later larval stage prior to pupation, empty their digestive track and disperse away from the food source in search of a dry and protected place to pupate (Sheppard et al., 1994). Pupae also require about two weeks to develop.

\section{Bioconversion of organic wastes via the black soldier fly farming}

The BSF larvae are voracious consumers of organic wastes, a detailed discussion of which is provided in a recent review by Gold et al. (2018). Studies have demonstrated that BSF larvae can consume a wide variety of organic resources ranging from food waste, agri-industry co-products, animal waste to meat-based products. Bonelli et al. (2019) described the morphological features and physiological functions of the midgut of the BSF larvae that enables BSF larvae to digest these diverse organic resources. In summary, the midgut of the BSF larvae has three different regions with distinct luminal $\mathrm{pH}$ : the acidic $(\mathrm{pH}=6.0)$ anterior midgut, strongly acidic $(\mathrm{pH}=2.0)$ middle midgut, and alkaline $(\mathrm{pH}=8.5)$ posterior midgut. Such variation in luminal $\mathrm{pH}$ along the midgut plays crucial roles in enzyme activity, nutrient solubility, detoxification of compounds ingested with substrate, and shaping gut microbiota. The ingested polysaccharides and some lipids are digested in the anterior midgut by soluble amylases and lipases, respectively, while remaining lipids and protein are mainly digested in the posterior midgut by the actions of lipases and endo- and exo-peptidases, respectively. The alkaline $\mathrm{pH}$ in the posterior midgut also enables BSF larvae to grow in substrates rich in tannins and other secondary metabolites that bind to proteins (i.e., 
enzymes) at low pH ranges. The high activity of lysozyme and strongly acidic $\mathrm{pH}$ in the middle midgut is believed to be responsible for inactivation of pathogens ingested with substrates (Bonelli et al., 2019). In all instances, the composition of the substrates must be considered, which may have a major impact on BSF development, survival, nutritional composition, and the substrate bioconversion rate. For example, depending on the substrate type, development can take between two weeks to several months and the resulting larvae can have protein contents ranging from 10 to $40 \%$ of body weight (Oonincx et al., 2015). The same variability also occurs with both fat content and composition of resulting BSF larvae reared on different substrates.

Due to such variability, further research is needed to understand the effect of substrate pretreatment prior to feeding on nutritional composition of BSF biomass. For example, particle size and microbial community can be manipulated to enhance BSF larvae development. More specifically, large particle size (i.e., whole vegetables or meat products) are challenging for the BSF to digest (Brits, 2017). Size reduction of the substrates prior to introducing it to the BSF larvae allows larvae to access nutrients more effectively. Also pretreating the substrate with chemicals (Isibika et al., 2019), bacteria (Isibika et al., 2019; Rehman et al., 2019; Somroo et al., 2019) or fungi (Gao et al., 2019; Isibika et al., 2019) may facilitate bioconversion. Similarly, selected microbes are also known to enhance (i.e., accelerate) BSF larvae development (Rehman et al., 2019; Yu et al., 2011).

\subsection{Conversion efficiency of organic resources}

The organic (substrate) reduction and bioconversion rate of organic resources into BSF biomass are summarized in Table 1. Studies have reported variation in substrate reduction and bioconversion rate, not only with substrate type (Gold et al., 2020), but also with BSF strain, feeding rate, and larval density among others. Thus, Bosch et al. (2020) have discussed and proposed procedures for standardizing the bioconversion studies using BSF larvae. For example, the BSF larvae of the Wuhan (China) strain not only weighed more than the Guanghzhou (China) and Texas (USA) strains ( $14.4 \%$ and $37.0 \%$, respectively), but also processed comparatively more substrate (Zhou et al., 2013a) (Table 1). Thus, selecting and maintaining the appropriate strain is critical in achieving improved substrate conversion and BSF biomass yield. In general, BSF colonies developed from local strains would result in better substrate bioconversion and minimize the risk of colony collapse (Zhou et al., 2013a). An extensive study on any variation in nutrient composition of different strains of the BSF larvae reared on a similar substrate is currently lacking.

Other factors that significantly affect substrate conversion include larval density, feeding rate, and feeding frequency (Banks et al., 2014; Diener et al., 2011, 2009; Parra Paz et al., 2015). To some extent, increasing the feeding rate results in faster growth and higher BSF biomass production, but adversely affects the waste reduction and biomass conversion rate (Banks et al., 2014; Diener et al., 2009). Conversely, a combination of high larval density and high feeding rate negatively affects the waste reduction and relative growth rate of the larvae (Parra Paz et al., 2015). Since the bioconversion of organic waste is mainly governed by the nutrient profile and composition of the organic waste, the feeding rate and larval density that result in a good balance between waste reduction and bioconversion rate vary with waste type. Moreover, the larval density and feeding rate vary with the goal of the bioconversion process. If the primary goal is organic waste valorization, a lower feeding rate and higher larval density are recommended; if the primary goal is BSF biomass production, a relatively higher feeding rate is preferred.
Studies have successfully explored co-treatment of organic wastes with BSF larvae and synergistic microorganisms to improve bioconversion (Abduh et al., 2017; Isibika et al., 2019; Mazza et al., 2020; Rehman et al., 2019; Somroo et al., 2019; Xiao et al., 2018b; Yu et al., 2011; Zheng et al., 2012). For example, Xiao et al. (2018b) observed improvement in BSF larvae weight, bioconversion rate, and substrate reduction by $16 \%, 13 \%$, and $13 \%$, respectively, while co-treating chicken manure with Bacillus subtilis and BSF larvae compared to treatment with BSF larvae alone. Positive effects on substrate conversion, larval weight gain, substrate reduction, and nutrient accumulation in BSF biomass have also been reported when chicken manure was co-treated with microbes isolated from eggs and larval gut of BSF (Mazza et al., 2020). Similarly, Somroo et al. (2019) reported improvement in BSF larval yield, substrate reduction, and bioconversion rate by $37 \%, 14 \%$, and $38 \%$, respectively, while co-treating soybean curd residue with Lactobacillus buchneri compared to mono-treatment with BSF larvae alone. Fermentation with fungi or supplementing exogenous microbes was also reported to improve the bioconversion and feed conversion ratio of lignocellulose-rich substrate such as rice straw (Zheng et al., 2012a), corn stover (Gao et al., 2019), banana peel (Isibika et al., 2019), and mixture of dairy manure and chicken manure (Rehman et al., 2019). The improved performance during cotreatment is attributed to the exo-enzymes, small molecules (e.g., simple sugars, peptides, and short-chain fatty acids), and nutrients produced by the synergistic microorganisms that promote the growth and substrate consumption rate of the BSF larvae (Isibika et al., 2019; Xiao et al., 2018b). Thus, inoculating the substrate with synergistic microorganisms along with the BSF larvae improves the bioconversion of organic wastes into BSF biomass. Since temporal and spatial variations exist in the composition of organic wastes, identifying synergistic mixed microbial cultures effective over a wide range of wastes would likely to further improve the bioconversion of organic wastes into BSF biomass. However, the added microorganisms should not have any negative effect on the nutrient value of the BSF biomass including pathogenicity and generation of allergens or toxins among others.

\subsection{Bioconversion approaches}

Approaches adopted for mass production of BSF biomass vary globally. Broadly, the approach for BSF biomass production could be categorized into two groups; (i) systems based on natural oviposition and (ii) systems based on adult rearing and egg production.

Historically, the process relied on natural populations colonizing the organic waste and self-harvesting of BSF biomass (Sheppard et al., 1994). The BSF biomass production system based on natural oviposition is discussed in detail elsewhere (Hem et al., 2008; Nyakeri et al., 2017a; Park, 2016; Rana et al., 2015; Sheppard et al., 1994). This system is applicable for small-scale farmers or home gardeners in regions with natural population of BSF (Kenis et al., 2018). Concerning abiotic factors, low- and middle-income countries in the tropical regions have favorable climate conditions for such system (i.e., BSF technology) to solve problems associated with a poorly-developed sanitation infrastructure (da Silva and Hesselberg, 2020). However, this approach is inefficient when considering the amount of organic wastes to be reduced and to reach the desired production levels for meeting the livestock, poultry, and aquaculture feeds demand. Moreover, such an approach is highly dependent on natural conditions to allow for the BSF to be active and colonize in organic wastes. In such case, colonization is unpredictable, resulting in lower production of BSF biomass, as well as spoilage of wastes, which may emanate odors. Furthermore, other pest species such as the house flies are likely to proliferate as well. 
Table 1

Substrate reduction, bioconversion, and feed conversion ratio of different organic wastes into BSF biomass.

\begin{tabular}{|c|c|c|c|c|}
\hline Substrate & $\begin{array}{l}\text { Substrate reduction } \\
(\% \text { of dry matter })^{\mathrm{A}}\end{array}$ & $\begin{array}{l}\text { Bioconversion } \\
(\%)^{\mathrm{B}}\end{array}$ & $\begin{array}{l}\text { Feed } \\
\text { conversion } \\
\text { ratio }^{\mathrm{C}}\end{array}$ & Reference \\
\hline Human feces & $25.2-54.6^{\mathrm{a}}$ & $1.6-22.9^{\mathrm{a}}$ & $2.0-33.9^{\mathrm{a}}$ & (Banks et al., 2014) \\
\hline Human feces & $39.1-48.6$ & $11.3-22.7$ & NA & $\begin{array}{l}\text { (Gold et al., 2020; Lalander et al., } \\
\text { 2019) }\end{array}$ \\
\hline Mixture of food waste and human feces (19:1) & $68.4-68.8$ & $18.9-19.0$ & NA & (Dortmans, 2015) \\
\hline Corn stover & 39.9 & 1.4 & 28.6 & (Wang et al., 2017) \\
\hline Fermented maize stover (mean $\pm \mathrm{SE}, \mathrm{n}=3$ ) & $48.4 \pm 0.0$ & $5.0^{\mathrm{b}}$ & $10.3^{\mathrm{b}}$ & (Gao et al., 2019) \\
\hline Fruit and vegetable waste & $46.7-60.0$ & $4.1-10.8$ & $9.3-12.5$ & $\begin{array}{l}\text { (Giannetto et al., 2019; Lalander et al., } \\
\text { 2019) }\end{array}$ \\
\hline Food waste (Mean $\pm S D, n=3$ ) & $55.3 \pm 4.1$ & $13.9 \pm 0.3$ & NA & (Lalander et al., 2019) \\
\hline Canteen waste (Mean $\pm S D, n>3$ ) & $37.9 \pm 3.8$ & $15.3 \pm 2.1$ & NA & (Gold et al., 2020) \\
\hline Vegetable canteen waste (Mean $\pm S D, n>3$ ) & $58.4 \pm 1.4$ & $22.7 \pm 1.1$ & NA & (Gold et al., 2020) \\
\hline Kitchen waste (Mean \pm SE, $n=3$ ) & $56.0 \pm 1.4$ & $18.2 \pm 0.2$ & NA & (Cai et al., 2017) \\
\hline Municipal organic waste & 68.0 & 11.8 & $5.8^{\mathrm{b}}$ & (Diener et al., 2011) \\
\hline Wheat-mill by-products (Mean $\pm \mathrm{SD}, \mathrm{n}>3$ ) & $56.4 \pm 1.2$ & $14.9 \pm 0.3$ & NA & (Gold et al., 2020) \\
\hline Poultry slaughterhouse waste (Mean $\pm S D, n>3$ ) & $30.7 \pm 4.7$ & $13.4 \pm 0.5$ & NA & (Gold et al., 2020) \\
\hline Abattoir waste (Mean $\pm S D, n=3$ ) & $46.3 \pm 2.9$ & $15.2 \pm 1.6$ & NA & (Lalander et al., 2019) \\
\hline $\begin{array}{l}\text { Mixture of abattoir waste and fruit and vegetable waste }(1: 1) \\
\quad(\text { Mean } \pm S D, n=3)\end{array}$ & $61.1 \pm 10.7$ & $14.2 \pm 1.9$ & NA & (Lalander et al., 2019) \\
\hline Mushroom root waste (Mean, $\mathrm{n}=3$ ) & $37.3-42.3$ & $5.4-5.6$ & NA & (Cai et al., 2017) \\
\hline Mixture of mushroom root waste and kitchen waste & $40.1-47.9$ & $10.2-15.3$ & NA & (Cai et al., 2017) \\
\hline Primary sludge (Mean $\pm S D, n=3$ ) & $63.3 \pm 1.9$ & $2.3 \pm 0.1$ & NA & (Lalander et al., 2019) \\
\hline Undigested sludge (Mean $\pm S D, n=3$ ) & $49.2 \pm 3.7$ & $2.2 \pm 0.2$ & NA & (Lalander et al., 2019) \\
\hline Digested sludge (Mean $\pm S D, n=3)$ & $13.2 \pm 0.8$ & $0.2 \pm 0.0$ & NA & (Lalander et al., 2019) \\
\hline $\begin{array}{l}\text { Mixture of pig manure, dog food, and human feces (4:4:2) } \\
\quad(\text { Mean } \pm S D, n=3 \text { ) }\end{array}$ & $55.1 \pm 0.3$ & $11.8 \pm 0.3$ & NA & (Lalander et al., 2015) \\
\hline Soybean curd residue & $49.0-72.4$ & $5.0-11.7$ & $6.2-9.8$ & $\begin{array}{l}\text { (Rehman et al., 2017; Somroo et al., } \\
\text { 2019) }\end{array}$ \\
\hline $\begin{array}{l}\text { Soybean curd residues + Bacteria (Lactobacillus buchneri) } \\
\quad(\text { Mean } \pm \mathrm{SE}, \mathrm{n}=3)\end{array}$ & $55.7 \pm 0.9$ & $6.9 \pm 0.3$ & $8.0 \pm 0.3$ & (Somroo et al., 2019) \\
\hline Fresh dairy manure & 53.1 & $12.2^{\mathrm{c}}$ & $4.4^{\mathrm{c}}$ & (Li et al., 2011b) \\
\hline Cattle manure & $12.7-43.2$ & $3.8-6.3$ & $4.2-10.3$ & $\begin{array}{l}\text { (Gold et al., 2020; Rehman et al., } \\
\text { 2017a, 2017b) }\end{array}$ \\
\hline Poultry manure & $35.8-60.0$ & $7.1-10.2$ & 5.6 & $\begin{array}{l}\text { (Lalander et al., 2019; Rehman et al., } \\
\text { 2017; Xiao et al., 2018b) }\end{array}$ \\
\hline Mixtures of dairy manure and soybean curd residues & $32.4-68.3$ & $9.7-15.2$ & $3.4-4.4$ & (Rehman et al., 2017) \\
\hline Mixtures of dairy manure and chicken manure & $44.3-53.4$ & $5.88-7.9$ & $6.6-7.5$ & (Rehman et al., 2017) \\
\hline Chicken manure + Bacillus subtilis (Mean $\pm \mathrm{SE}, \mathrm{n}=3$ ) & $40.5 \pm 0.8$ & $11.5 \pm 0.2$ & NA & (Xiao et al., 2018b) \\
\hline Swine manure (Mean $\pm S E, n=3$ ) & $\begin{array}{l}53.4 \pm 0.3^{\mathrm{I}} \\
28.8 \pm 0.2^{\mathrm{II}} \\
49.7 \pm 0.4^{\mathrm{III}}\end{array}$ & NA & NA & (Zhou et al., 2013a) \\
\hline Chicken manure (Mean $\pm \mathrm{SE}, \mathrm{n}=3$ ) & $\begin{array}{l}61.7 \pm 0.2^{\mathrm{I}} \\
31.8 \pm 0.3^{\mathrm{II}} \\
56.8 \pm 0.4^{\mathrm{III}}\end{array}$ & NA & NA & (Zhou et al., 2013a) \\
\hline Dairy manure (Mean $\pm S E, n=3$ ) & $\begin{array}{l}57.8 \pm 0.7^{\mathrm{I}} \\
53.2 \pm 0.5^{\mathrm{II}} \\
25.8 \pm 0.2^{\mathrm{III}}\end{array}$ & NA & NA & (Zhou et al., 2013a) \\
\hline $\begin{array}{l}\text { Mixture of dairy manure and chicken manure (2:3) (Mean } \pm \text { SE, } \\
\quad n=3 \text { ) }\end{array}$ & $42.0 \pm 0.5$ & $6.8 \pm 0.0$ & $6.1 \pm 0.1$ & (Rehman et al., 2019) \\
\hline $\begin{array}{l}\text { Mixture of dairy manure and chicken manure }(2: 3)+\text { Paenibacillus } \\
\text { polymyxa strain } \mathrm{KMZ}(\text { Mean } \pm \mathrm{SE}, \mathrm{n}=3)\end{array}$ & $41.8 \pm 0.5$ & $8.1 \pm 0.3$ & $5.2 \pm 0.1$ & (Rehman et al., 2019) \\
\hline $\begin{array}{l}\text { Mixture of dairy manure and chicken manure }(2: 3)+\text { Paenibacillus } \\
\text { polymyxa strain ZRO2 (Mean } \pm \mathrm{SE}, \mathrm{n}=3 \text { ) }\end{array}$ & $47.3 \pm 0.5$ & $9.5 \pm 0.2$ & $5.0 \pm 0.2$ & (Rehman et al., 2019) \\
\hline $\begin{array}{l}\text { Mixture of dairy manure and chicken manure }(2: 3)+\text { Bacillus } \\
\text { strains SMO1 (Mean } \pm \mathrm{SE}, \mathrm{n}=3 \text { ) }\end{array}$ & $45.5 \pm 0.6$ & $8.9 \pm 0.3$ & $5.1 \pm 0.2$ & (Rehman et al., 2019) \\
\hline $\begin{array}{l}\text { Mixture of dairy manure and chicken manure }(2: 3)+\text { Bacillus } \\
\text { strains SMO2 (Mean } \pm \text { SE, } n=3)\end{array}$ & $42.2 \pm 0.2$ & $9.1 \pm 0.3$ & $4.6 \pm 0.2$ & (Rehman et al., 2019) \\
\hline $\begin{array}{l}\text { Mixture of dairy manure and chicken manure }(2: 3)+\text { Bacillus } \\
\text { strains MRO2 (Mean } \pm \mathrm{SE}, \mathrm{n}=3 \text { ) }\end{array}$ & $48.8 \pm 0.7$ & $10.8 \pm 0.0$ & $4.49 \pm 0.1$ & (Rehman et al., 2019) \\
\hline $\begin{array}{l}\text { Mixture of dairy manure and chicken manure }(2: 3)+\text { Bacillus } \\
\text { strains MRO4 (Mean } \pm \text { SE, } n=3 \text { ) }\end{array}$ & $34.5 \pm 1.0$ & $9.0 \pm 0.1$ & $4.2 \pm 0.2$ & (Rehman et al., 2019) \\
\hline
\end{tabular}

A Substrate reduction (\% of dry matter) $=$ [(Weight of substrate added - Weight of residue $) /$ Weight of substrate added $] \times 100 \%$; ${ }^{\mathrm{B}}$ Bioconversion $(\%)=($ Weight of larval

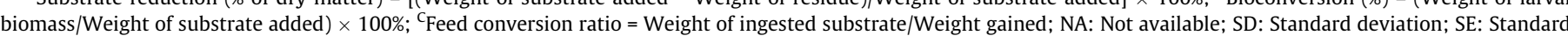

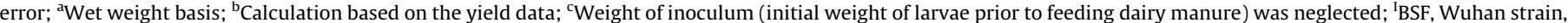

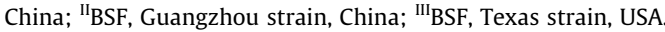

In case of the system based on adult rearing and egg production, a BSF colony needs to be maintained to ensure a regular supply of BSF eggs and simultaneously facilitating the waste bioconversion (Sheppard et al., 2002). This approach allows eggs to be produced in-house for bioconversion of wastes by the resulting larvae. Thus, adult rearing and egg production is a critical component of this type of BSF biomass production system (Kenis et al., 2018). The details of maintaining the colony to ensure a regular supply of eggs can be found elsewhere (Bertinetti et al., 2019; Caruso et al., 2013; Cortes Ortiz et al., 2016; Dortmans et al., 2017; Hoc et al., 2019; Sheppard et al., 2002). More recent BSF biomass production systems rely on maintaining a BSF colony. Such system is commonly 


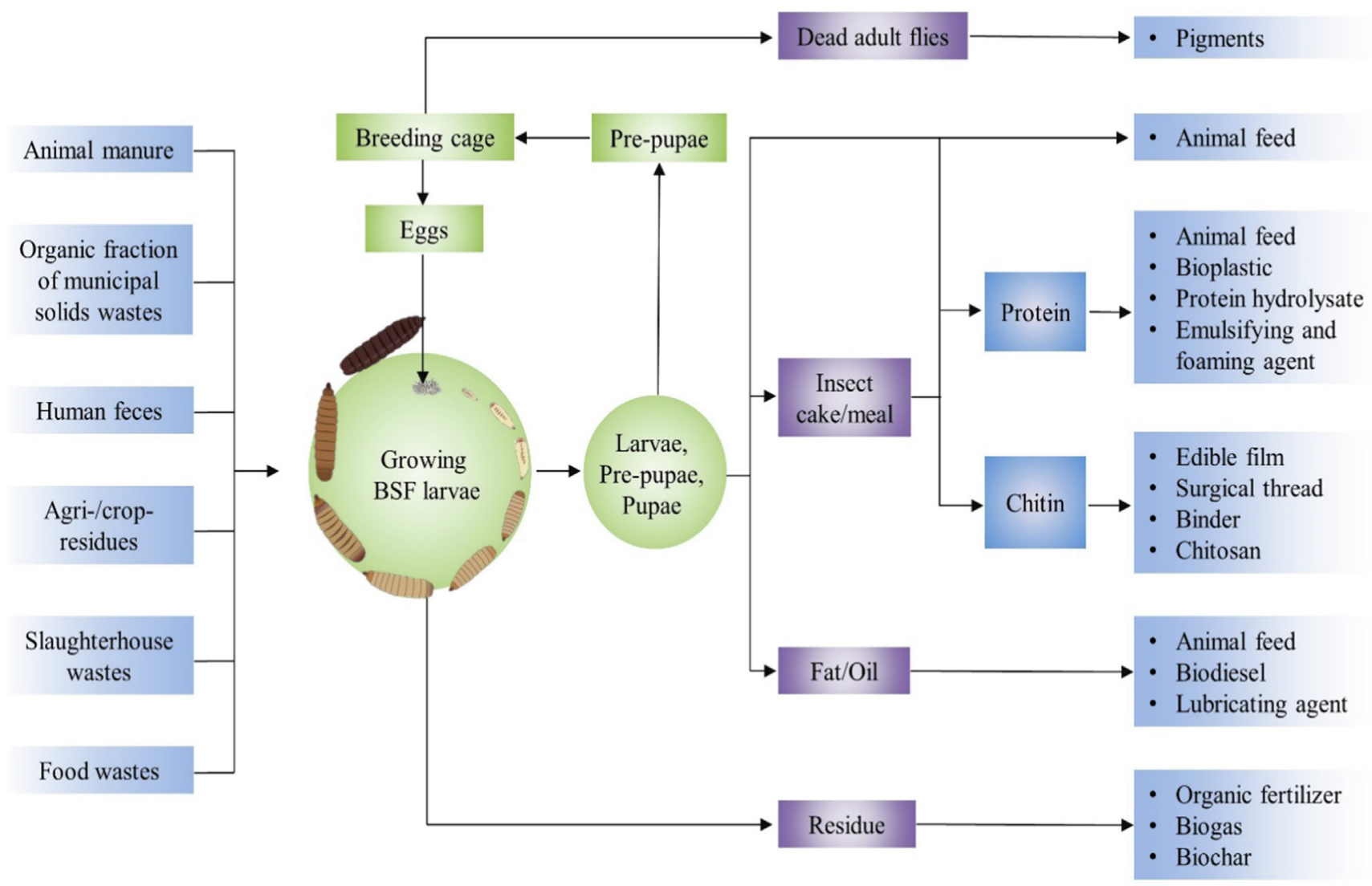

Fig. 2. A schematic of a BSF-based biorefinery for producing value-added products with concurrent valorization of organic bioresources.

used as it allows for somewhat predictable input (organic waste) and output (BSF biomass production). However, the major limitation of this approach is the space requirement for a commercialscale production. This is because given the size of an adult cage $[1.2 \mathrm{~m}(\mathrm{H}) \times 1.2 \mathrm{~m}(\mathrm{~W}) \times 2.4 \mathrm{~m}(\mathrm{~L})]$, and its egg production capability (i.e., approximately $10 \mathrm{~g} /$ day) (Tomberlin, unpublished data), the number of cages needed to feed an industrial BSF complex could result in a massive spatial footprint, which may limit such facilities to locate outside of urban areas. For example, as a rule of thumb, a facility treating one ton of organic waste per day will require about $50 \mathrm{~m}^{2}$ area for maintaining the BSF colony (i.e., BSF nursery) (Dortmans et al., 2017). Furthermore, such large-scale facilities are susceptible to environmental variability (e.g., temperature, humidity), which can impact egg production and hatching. One approach to overcome the footprint requirement is to develop vertical system where cages can be stacked on top of one another.

\subsection{Black soldier fly-based biorefinery}

Like a petroleum refinery that produces different fuels and products from crude petroleum, BSF farming could also serve as a model biorefinery for valorizing various organic resources into feed, fuel, fertilizer, and other bio-based products. A BSF-based biorefinery (Fig. 2), a practical adoption of a circular economy approach, could potentially increase the economic viability of the BSF technology and minimize the environmental footprint of the aforementioned BSF-derived products.

As previously stated, BSF can convert diverse organic resources into protein- and fat-rich biomass (Banks et al., 2014; Diener et al., 2011; Gao et al., 2019; Lalander et al., 2019; Rehman et al., 2009, 2017; Somroo et al., 2019; Surendra et al., 2016; Wang et al., 2017; Xiao et al., 2018b; Zhou et al., 2013), which could replace a significant amount of conventional proteins in animal diets, without significantly affecting the performance of the animal (see sections 4 and 5 for details). Biodiesel from BSF fat (Feng et al., 2018, 2020; Ishak and Kamari, 2019; Kamarulzaman et al., 2019; Leong et al., 2016; Li et al., 2011a, 2011b, 2015, Nguyen et al., 2018, 2020; Pang et al., 2019; Su et al., 2019; Surendra et al., 2016; Wang et al., 2017; Zheng et al., 2012; Rehman et al., 2018) could be one of the major products of a BSF-based biorefinery. Since BSF biomass is rich in fat (21 to $40 \%$ of dry matter), this fat could be extracted and converted into biodiesel via transesterification. Studies have explored different techniques in extracting fat from BSF biomass (Feng et al., 2018, 2020; Mai et al., 2019; Matthäus et al., 2019; Ravi et al., 2019; Smets et al., 2020; Soetemans et al., 2019; Su et al., 2019). In general, BSF fat is rich in medium chain saturated fatty acids and low in polyunsaturated fatty acids, which could produce biodiesel with low viscosity and high oxidative stability (Surendra et al., 2016; Zheng et al., 2012a). Moreover, studies have shown that properties of the biodiesel produced from BSF fat are within the international standard for biodiesel including the American Society for Test and Materials (ASTM) D6751 (Ishak and Kamari, 2019; Su et al., 2019) and the European standard EN 14,214 (Ishak and Kamari, 2019; Li et al., 2011; Nguyen et al., 2018; Su et al., 2019). In biodiesel production, the feedstock accounts for majority of the production cost (up to 75\%) (Canakci and Sanli, 2008). Thus, a large-scale production of fat-rich BSF biomass from low-value organic resources could substantially reduce the production cost of biodiesel.

The residual substrate following harvest of BSF biomass could be used as an organic fertilizer (Bloukounon-Goubalan et al., 2019; Cai et al., 2017; Choi et al., 2009; Liu et al., 2019; Rosmiati et al., 2017). The nutrient profile of the residue is mainly governed by substrate fed to BSF larvae. Studies have reported increased 
Table 2

Proximate composition of BSF biomass reared on different organic wastes (Unit: \% of dry matter; *: \% of lyophilized prepupae matter).

\begin{tabular}{|c|c|c|c|c|c|c|c|c|c|}
\hline Substrate & $\begin{array}{l}\text { Growth } \\
\text { stage }\end{array}$ & $\begin{array}{l}\text { Crude } \\
\text { protein }\end{array}$ & Crude fat & $\begin{array}{l}\text { Carbohyd- } \\
\text { rate }\end{array}$ & $\begin{array}{l}\text { Crude } \\
\text { fiber }\end{array}$ & Ash & $\begin{array}{l}\text { Gross energy } \\
(\mathrm{Kcal} / \mathrm{kg})\end{array}$ & Chitin & Reference \\
\hline Chicken feed & Prepupae & 41.2 & 33.6 & NA & NA & 10.0 & NA & 6.2 & (Spranghers et al., 2017) \\
\hline Biogas digestate & Prepupae & 42.2 & 21.8 & NA & NA & 19.7 & NA & 5.6 & (Spranghers et al., 2017) \\
\hline Vegetable waste & Prepupae & 39.9 & 37.1 & NA & NA & 9.6 & NA & 5.7 & (Spranghers et al., 2017) \\
\hline Restaurant waste & Prepupae & 43.1 & 38.6 & NA & NA & 2.7 & NA & 6.7 & (Spranghers et al., 2017) \\
\hline Cafeteria waste (Mean $\pm S D, n=3$ ) & Prepupae & $43.7 \pm 0.6$ & $31.8 \pm 0.3$ & $12.3 \pm 0.3$ & $10.1 \pm 0.2$ & $6.0 \pm 0.0$ & $5751.7 \pm 52.4$ & NA & (Surendra et al., 2016) \\
\hline Vegetable and fruit waste $(7: 3)$ & Larvae & 41.9 & 26.3 & NA & NA & 13.0 & NA & 6.2 & (Meneguz et al., 2018) \\
\hline $\begin{array}{l}\text { Vegetable and fruit waste }(17: 3) \\
\quad(\text { Mean } \pm S D, n=3)\end{array}$ & Larvae & $36.7 \pm 2.7$ & $33.0 \pm 0.2$ & NA & NA & $5.0 \pm 0.0$ & NA & NA & (Giannetto et al., 2019) \\
\hline $\begin{array}{l}\text { Vegetable and fruit waste }(17: 3) \\
\quad(\text { Mean } \pm S D, n=3)\end{array}$ & Prepupae & $39.9 \pm 1.1$ & $30.8 \pm 0.2$ & NA & NA & $5.7 \pm 0.1$ & NA & NA & (Giannetto et al., 2019) \\
\hline Fruit waste & Larvae & $\begin{array}{l}30.7- \\
39.8\end{array}$ & $\begin{array}{l}40.7- \\
47.4\end{array}$ & NA & NA & $7.2-7.9$ & 27201.7 & 5.6 & $\begin{array}{l}\text { (Chun et al., 2019; } \\
\text { Meneguz et al., 2018) }\end{array}$ \\
\hline Fruit waste & Prepupae & 43.8 & 46.8 & NA & NA & 5.7 & 27825.2 & NA & (Chun et al., 2019) \\
\hline Winery by-product & Larvae & 34.4 & 32.2 & NA & NA & 14.6 & NA & 5.3 & (Meneguz et al., 2018) \\
\hline Brewery by-product & Larvae & 53.0 & 29.9 & NA & NA & 7.3 & NA & 1.4 & (Meneguz et al., 2018) \\
\hline $\begin{array}{l}\text { Brown algae (Ascophyllum nodosum) } \\
\quad(\text { Mean } \pm S D, n=4)\end{array}$ & Larvae & $41.3 \pm 1.1$ & $8.1 \pm 0.9$ & NA & NA & $15.8 \pm 0.7$ & NA & NA & (Liland et al., 2017) \\
\hline Food waste & Prepupae & $\begin{array}{l}42.0- \\
43.7\end{array}$ & $\begin{array}{l}35.0- \\
37.2\end{array}$ & NA & NA & $3.1-4.6$ & NA & NA & $\begin{array}{l}\text { (Salomone et al., 2017; } \\
\text { Wang et al., 2020) }\end{array}$ \\
\hline Pig manure* (Mean $\pm S E, n=3$ ) & Prepupae & $42.8 \pm 4.4$ & $36.5 \pm 3.9$ & NA & NA & $3.7 \pm 0.4$ & NA & NA & (Wang et al., 2020) \\
\hline Poultry manure* (Mean $\pm \mathrm{SE}, \mathrm{n}=3)$ & Prepupae & $41.7 \pm 4.0$ & $36.2 \pm 3.5$ & NA & NA & $3.8 \pm 0.4$ & NA & NA & (Wang et al., 2020) \\
\hline Cow manure* $($ Mean $\pm \mathrm{SE}, \mathrm{n}=3)$ & Prepupae & $41.2 \pm 2.1$ & $35.7 \pm 2.9$ & NA & NA & $4.9 \pm 0.5$ & NA & NA & (Wang et al., 2020) \\
\hline Fermented maize stover & Prepupae & 41.8 & 30.5 & NA & NA & 8.2 & NA & NA & (Gao et al., 2019) \\
\hline Cricket waste & Larvae & 37.1 & 38.7 & NA & NA & 9.4 & NA & NA & (Jucker et al., 2020) \\
\hline Locust waste & Larvae & 49.2 & 25.8 & NA & NA & 13.2 & NA & NA & (Jucker et al., 2020) \\
\hline
\end{tabular}

NA: Not available; SD: Standard deviation; SE: Standard error.

concentration of total phosphorus (Lalander et al., 2015) and ammonium nitrogen (Green and Popa, 2012; Lalander et al., 2015) in the residue following BSF larval composting, while the $\mathrm{pH}$ of the residue usually is within the optimum range for plant growth ( $\mathrm{pH}=7.0$ - 8.0) (Choi et al., 2009; Lalander et al., 2015). Moreover, studies have reported positive effects of chitin or its derivatives on plant (i.e., triggers growth and induces defense) (Sharif et al., 2018; Sharp, 2013). Since the residue contains larvae or pupae exuviae and dead imago, rich sources of chitin (Purkayastha and Sarkar, 2019), the residue could also trigger plant growth and induce plant defense. However, currently the studies exploring such aspects of residue is lacking. Additionally, the insect compost as a growth media could replace a substantial amount of peat and synthetic fertilizer in soilless agriculture (Setti et al., 2019). As discussed in the section 7, the bioconversion of organic wastes using BSF larvae reduces pathogen levels and degrades pesticides and pharmaceuticals, thereby preventing the spread of such contaminants into the environment. Additionally, the BSF bioaccumulate heavy metals such as cadmium (Cd) (Biancarosa et al., 2018; Purschke et al., 2017; Tschirner and Simon, 2015; van der Fels-Klerx et al., 2016a), lead (Pb) (Purschke et al., 2017; Tschirner and Simon, 2015; van der Fels-Klerx et al., 2016a), and arsenic (As) (Biancarosa et al., 2018, 2019), thereby improving the value of the residual compost as an organic fertilizer. Moreover, the residual biomass could be used as a substrate for producing biofuel and bio-based products following biochemical (e.g., anaerobic digestion (Bulak et al., 2020) and/or composting) and thermochemical (e.g., pyrolysis, hydrothermal carbonization) conversion processes. The downstream applications of the insect compost, however, depend on its composition, which is governed by the characteristics of the original organic waste fed to BSF larvae.

In addition to producing feed, fuel, and fertilizer, the BSF-based biorefinery has huge potential in producing other high-value products. For example, chitin, a polymer of glucosamine, is a major byproduct of BSF biomass processing as it constitutes up to $7 \%$ of BSF biomass on dry matter basis (Meneguz et al., 2018; Spranghers et al., 2017). Chitin and its derivative, chitosan, have many industrial applications such as a binder (in dyes), edible films, industrial membranes, biodegradable surgical thread, and a fining agent (in winemaking). Moreover, based on the physicochemical characteristics of the BSF-derived chitin, studies have suggested its potential applications in tissue engineering, textile industry, and as an adsorbent in water and wastewater treatment (Purkayastha and Sarkar, 2019; Wasko et al., 2016). Moreover, economicallyimportant bioproducts such as protein hydrolysates (Firmansyah and Abduh, 2019), bioplastic (Barbi et al., 2019), and natural pigments (e.g., melanin and ommochromes) (Ushakova et al., 2019) could also be derived from BSF biomass. Studies have also explored BSF biomass as a potential source of industrially important enzymes (e.g., trypsin, chymotrypsin, ligninase, and cellulase) (Bonelli et al., 2019; Kim et al., 2011; Lee et al., 2014; Muller et al., 2017; Park et al., 2012) and antimicrobial peptides. The successful development of a BSF-based biorefinery, however, will require a large-scale production of BSF biomass, which in turn will require mechanization and automation of rearing, harvesting, and processing.

\section{Nutrient composition of black soldier fly biomass}

The nutrient profile of BSF biomass is summarized in Tables 2 to 5 . BSF biomass is rich in protein, fat and minerals, and has comparatively low fiber content. As shown in Table 2, crude protein and fat contents of BSF biomass vary from 30 to $52 \%$ and 21 to $40 \%$ of dry matter, respectively.

The crude protein content in BSF biomass compares well with the most common plant protein source: soybean meal, while the gross energy value of BSF biomass is usually higher than that of common energy sources in animal diets such as corn meal and wheat meal (Surendra et al., 2016). The crude protein content of defatted BSF biomass is higher than that of soybean meal and is comparable to fish meal (Surendra et al., 2016). The extracted fat from BSF biomass has other potential applications such as a feedstock for biodiesel (as discussed in section 3.3) or biobased lubricant (Alipour et al., 2019). 
Table 3

Amino acids profile of the BSF biomass reared on different organic wastes (Unit: *: \% of dry matter; **: \% of crude protein; ***: \% of lyophilized prepupa matter)

\begin{tabular}{|c|c|c|c|c|c|c|c|c|c|c|c|c|c|c|c|c|}
\hline \multicolumn{17}{|l|}{ Essential amino acids } \\
\hline \multirow{2}{*}{$\begin{array}{l}\text { Substrate } \\
\text { Chicken feed* }\end{array}$} & $\begin{array}{l}\text { Growth } \\
\text { stage }\end{array}$ & \multicolumn{2}{|c|}{ Arginine } & Histidine & Isoleucine & Leucine & Lysine & \multicolumn{2}{|c|}{ Methionine } & \multicolumn{2}{|c|}{ Phenylalanine } & \multicolumn{2}{|c|}{ Threonine } & Tryptophan & n Valine & References \\
\hline & Prepupae & 2.03 & & 1.36 & 1.72 & 2.86 & 2.34 & 0.76 & & 1.70 & & 1.64 & & 0.67 & 2.41 & $\begin{array}{l}\text { (Spranghers et al., } \\
\text { 2017) }\end{array}$ \\
\hline Digestate* & Prepupae & 2.03 & & 1.35 & 1.84 & 2.95 & 2.57 & 0.87 & & 1.87 & & 1.68 & & 0.62 & 2.49 & $\begin{array}{l}\text { (Spranghers et al., } \\
\text { 2017) }\end{array}$ \\
\hline Vegetable waste* & Prepupae & 2.00 & & 1.24 & 1.73 & 2.80 & 2.26 & 0.76 & & 1.63 & & 1.54 & & 0.58 & 2.48 & $\begin{array}{l}\text { (Spranghers et al., } \\
\text { 2017) }\end{array}$ \\
\hline Restaurant waste* & Prepupae & 1.99 & & 1.38 & 1.91 & 3.06 & 2.30 & 0.71 & & 1.64 & & 1.62 & & 0.54 & 2.82 & $\begin{array}{l}\text { (Spranghers et al., } \\
\text { 2017) }\end{array}$ \\
\hline Cafeteria waste* (Mean $\pm S D, n=3$ ) & Prepupae & $2.22 \pm$ & $=0.08$ & $1.69 \pm 0.04$ & $04 \quad 1.51 \pm 0.06$ & $2.34 \pm 0.05$ & $2.19 \pm 0.07$ & 0.88 & $=0.02$ & $1.50 \pm$ & 0.07 & $1.48 \pm$ & 0.07 & NA & $2.42 \pm 0.06$ & (Surendra et al., 2016) \\
\hline Abattoir waste** & Larvae & 4.96 & & 3.53 & 4.67 & 6.84 & 6.40 & 1.77 & & 3.59 & & 3.70 & & 1.67 & 6.32 & (Lalander et al., 2019) \\
\hline Poultry manure** & Larvae & 4.92 & & 3.09 & 4.10 & 6.61 & 7.07 & 2.05 & & 4.20 & & 3.83 & & 1.62 & 5.97 & (Lalander et al., 2019) \\
\hline $\begin{array}{l}\text { Poultry manure } * * * \text { (Mean } \pm S E \text {, } \\
\quad n=3 \text { ) }\end{array}$ & Prepupae & $2.06 \pm$ & $=0.21$ & $1.39 \pm 0.11$ & $11 \quad 1.77 \pm 0.18$ & $2.81 \pm 0.25$ & $2.41 \pm 0.23$ & 0.79 & $=0.05$ & $1.83 \pm$ & 0.16 & $1.93 \pm$ & 0.17 & $0.75 \pm 0.06$ & $1.84 \pm 0.18$ & (Wang et al., 2020) \\
\hline Human feces** & Larvae & 5.10 & & 3.30 & 4.67 & 7.00 & 6.17 & 1.85 & & 4.42 & & 3.76 & & 1.69 & 6.59 & (Lalander et al., 2019) \\
\hline Food waste ${ }^{* *}$ & Larvae & 4.89 & & 2.91 & 4.13 & 6.79 & 8.25 & 1.84 & & 4.03 & & 3.86 & & 1.38 & 5.84 & (Lalander et al., 2019) \\
\hline Food waste $* * *($ Mean $\pm S E, n=3)$ & Prepupae & $2.09 \pm$ & $=0.20$ & $1.42 \pm 0.11$ & $11 \quad 1.84 \pm 0.16$ & $2.86 \pm 0.24$ & $2.40 \pm 0.21$ & 0.80 & $=0.06$ & $1.81 \pm$ & 0.18 & $1.91 \pm$ & 0.15 & $0.78 \pm 0.07$ & $7 \quad 1.97 \pm 0.18$ & (Wang et al., 2020) \\
\hline Pig manure ${ }^{* * *}($ Mean $\pm S E, n=3)$ & Prepupae & $2.11 \pm$ & $=0.17$ & $1.39 \pm 0.10$ & $10 \quad 1.83 \pm 0.14$ & $2.81 \pm 0.22$ & $2.46 \pm 0.19$ & 0.81 & $=0.06$ & $1.85 \pm$ & 0.15 & $1.95 \pm$ & 0.10 & $0.80 \pm 0.06$ & $1.95 \pm 0.16$ & (Wang et al., 2020) \\
\hline Cow manure ${ }^{* * *}($ Mean $\pm S E, n=3)$ & Prepupae & $2.01 \pm$ & $=0.16$ & $1.30 \pm 0.12$ & $12 \quad 1.75 \pm 0.15$ & $2.74 \pm 0.21$ & $2.34 \pm 0.22$ & 0.75 & $=0.04$ & $1.72 \pm$ & 0.15 & $1.84 \pm$ & 0.13 & $0.71 \pm 0.05$ & $5 \quad 1.92 \pm 0.21$ & (Wang et al., 2020) \\
\hline Fruit and vegetable waste ${ }^{* *}$ & Larvae & 4.54 & & 2.63 & 4.30 & 6.67 & 5.14 & 1.53 & & 3.45 & & 3.46 & & 1.40 & 5.95 & (Lalander et al., 2019) \\
\hline Primary sludge** & Larvae & 4.94 & & 2.97 & 4.50 & 6.55 & 5.94 & 1.81 & & 3.76 & & 3.60 & & 1.65 & 6.16 & (Lalander et al., 2019) \\
\hline Undigested sludge** & Larvae & 4.94 & & 3.13 & 4.47 & 7.56 & 6.42 & 1.83 & & 3.99 & & 3.70 & & 1.71 & 6.08 & (Lalander et al., 2019) \\
\hline Digested sludge** & Larvae & 4.49 & & 1.98 & 3.90 & 6.25 & 6.17 & 1.81 & & 3.73 & & 3.31 & & 1.79 & 5.28 & (Lalander et al., 2019) \\
\hline Household organic waste* & Larvae & 1.94 & & 1.32 & 1.57 & 2.59 & 2.22 & 0.58 & & 1.51 & & 1.42 & & 0.53 & 2.25 & (Kawasaki et al., 2019) \\
\hline Household organic waste* & Prepupae & 2.20 & & 1.30 & 1.71 & 2.81 & 2.51 & 0.74 & & 1.69 & & 1.55 & & 0.66 & 2.38 & (Kawasaki et al., 2019) \\
\hline Brown $_{\text {algae }}^{* *}($ Mean $\pm S D, n=4)$ & Larvae & 6.50 & & 2.70 & 3.80 & 6.20 & 5.60 & 1.40 & & 3.20 & & 3.90 & & NA & 5.50 & (Liland et al., 2017) \\
\hline \multicolumn{17}{|l|}{ Non-essential amino acids } \\
\hline Substrate & \multicolumn{2}{|c|}{ Growth stage } & \multicolumn{2}{|c|}{ Alanine } & Aspartic acid & Cysteine & \multicolumn{2}{|c|}{ Glutamic acid } & Glycil & & Proline & & Seri & & Tyrosine & References \\
\hline Chicken feed* & Prepupae & & 2.52 & & 3.78 & 0.25 & 4.19 & & 2.26 & & 2.25 & & 1.66 & & NA & (Spranghers et al., 2017) \\
\hline Digestate* & Prepupae & & 2.43 & & 3.36 & 0.24 & 3.96 & & 2.26 & & 2.21 & & 1.55 & & NA & (Spranghers et al., 2017) \\
\hline Vegetable waste* & Prepupae & & 2.42 & & 3.59 & 0.21 & 4.13 & & 2.22 & & 2.14 & & 1.50 & & NA & (Spranghers et al., 2017) \\
\hline Restaurant waste* & Prepupae & & 2.78 & & 3.69 & 0.22 & 4.58 & & 2.52 & & 2.51 & & $1.5 \mathrm{~s}$ & & NA & (Spranghers et al., 2017) \\
\hline Cafeteria waste* (Mean $\pm S D, n=3$ ) & Prepupae & & 2.68 & \pm 0.19 & $2.64 \pm 0.06$ & $1.12 \pm 0.05$ & $2.85 \pm 0.02$ & & $2.46=$ & \pm 0.10 & $2.11 \pm$ & 0.02 & 1.53 & \pm 0.01 & $2.37 \pm 0.02$ & (Surendra et al., 2016) \\
\hline Abattoir waste*** & Larvae & & 5.72 & & 9.28 & 0.60 & 10.00 & & 5.80 & & 5.06 & & 4.05 & & 8.62 & (Lalander et al., 2019) \\
\hline Poultry manure** & Larvae & & 5.65 & & 9.26 & 0.55 & 9.66 & & 5.41 & & 5.20 & & 3.84 & & 6.21 & (Lalander et al., 2019) \\
\hline Human feces** & Larvae & & 6.53 & & 9.00 & 0.67 & 10.59 & & 5.92 & & 5.79 & & 4.39 & & 10.88 & (Lalander et al., 2019) \\
\hline Food waste** & Larvae & & 5.93 & & 9.08 & 0.53 & 9.84 & & 5.27 & & 5.12 & & 4.13 & & 6.02 & (Lalander et al., 2019) \\
\hline Food waste ${ }^{* * *}($ Mean $\pm S E, n=3)$ & Prepupae & & 2.44 & \pm 0.21 & $3.70 \pm 0.34$ & $0.26 \pm 0.03$ & $4.18 \pm 0.29$ & & $2.29=$ & \pm 0.21 & $2.26 \pm$ & 0.18 & 1.92 & \pm 0.17 & NA & (Wang et al., 2020) \\
\hline Pig manure ${ }^{* * *}($ Mean $\pm S E, n=3)$ & Prepupae & & 2.31 & \pm 0.19 & $3.72 \pm 0.36$ & $0.28 \pm 0.02$ & $4.11 \pm 0.38$ & & $2.26=$ & \pm 0.18 & $2.27 \pm$ & 0.14 & 1.75 & \pm 0.14 & NA & (Wang et al., 2020) \\
\hline Poultry manure $^{* * *}($ Mean $\pm S E, n=3)$ & Prepupae & & 2.35 & \pm 0.21 & $3.61 \pm 0.29$ & $0.21 \pm 0.03$ & $4.07 \pm 0.41$ & & $2.24=$ & \pm 0.19 & $2.10 \pm$ & 0.16 & 1.72 & \pm 0.15 & NA & (Wang et al., 2020) \\
\hline Cow manure ${ }^{* * *}($ Mean $\pm \mathrm{SE}, \mathrm{n}=3)$ & Prepupae & & $2.32=$ & \pm 0.18 & $3.58 \pm 0.32$ & $0.24 \pm 0.01$ & $4.05 \pm 0.37$ & & $2.17=$ & \pm 0.20 & $2.17 \pm$ & 0.22 & 1.61 & \pm 0.11 & NA & (Wang et al., 2020) \\
\hline Fruit and vegetable waste ${ }^{* *}$ & Larvae & & 5.48 & & 8.06 & 0.50 & 9.54 & & 5.24 & & 5.28 & & 3.86 & & 5.50 & (Lalander et al., 2019) \\
\hline Primary sludge** & Larvae & & 5.57 & & 8.85 & 0.66 & 10.04 & & 5.60 & & 5.59 & & 4.06 & & 6.02 & (Lalander et al., 2019) \\
\hline Undigested sludge** & Larvae & & 5.66 & & 8.90 & 0.67 & 10.38 & & 5.55 & & 5.35 & & 4.25 & & 6.56 & (Lalander et al., 2019) \\
\hline Digested sludge** & Larvae & & 4.82 & & 8.26 & 0.65 & 9.57 & & 4.83 & & 4.65 & & 3.66 & & 6.26 & (Lalander et al., 2019) \\
\hline Household organic waste* & Larvae & & 2.45 & & 3.43 & 0.28 & 3.99 & & 1.90 & & 2.16 & & 1.62 & & 2.30 & (Kawasaki et al., 2019) \\
\hline Household organic waste* & Prepupae & & 2.28 & & 3.74 & 0.28 & 4.30 & & 2.11 & & 2.14 & & 1.70 & & 2.63 & (Kawasaki et al., 2019) \\
\hline Brown algae*** & Larvae & & 6.40 & & 8.30 & NA & 11.90 & & 4.30 & & 5.20 & & 4.30 & & 4.20 & (Liland et al., 2017) \\
\hline
\end{tabular}

NA: Not available; SD: Standard deviation; SE: Standard error. 
Table 4

Fatty acids profile of the of the BSF biomass reared on different organic wastes (Unit: \% w/w of fatty acid methyl ester)

\begin{tabular}{|c|c|c|c|c|c|c|c|c|c|c|c|c|c|c|}
\hline Substrate & $\begin{array}{l}\text { Growth } \\
\text { stage }\end{array}$ & $\begin{array}{l}\text { Capric } \\
\text { C10:0 }\end{array}$ & $\begin{array}{l}\text { Lauric } \\
\text { (C12:0) }\end{array}$ & $\begin{array}{l}\text { Myristic } \\
\text { (C14:0) }\end{array}$ & $\begin{array}{l}\text { Palmitic } \\
(\mathrm{C} 16: 0)\end{array}$ & $\begin{array}{l}\text { Palmitol-eic } \\
(\mathrm{C} 16: 1 \mathrm{n}-7)\end{array}$ & $\begin{array}{l}\text { Stearic } \\
(\mathrm{C} 18: 0)\end{array}$ & $\begin{array}{l}\text { Oleic } \\
(\mathrm{C} 18: 1 \mathrm{n}-9)\end{array}$ & $\begin{array}{l}\text { Linoleic } \\
(C 18: 2 n-6)\end{array}$ & $\begin{array}{l}\text { Linolenic } \\
\text { (C18:3n-3) }\end{array}$ & SFA & MUFA & PUFA & Reference \\
\hline Chicken feed & Prepupae & 1.4 & 57.4 & 7.3 & 9.7 & NA & 1.4 & 7.5 & NA & 0.7 & 77.4 & 10.0 & 12.6 & (Spranghers et al., 2017) \\
\hline Digestate & Prepupae & 1.2 & 43.7 & 6.9 & 10.1 & NA & 1.8 & 7.9 & NA & 0.8 & 64.8 & 19.1 & 9.6 & (Spranghers et al., 2017) \\
\hline Vegetable waste & Prepupae & 1.6 & 60.9 & 9.5 & 8.7 & NA & 1.1 & 5.7 & NA & 1.4 & 82.8 & 9.5 & 7.0 & (Spranghers et al., 2017) \\
\hline Restaurant waste & Prepupae & 2.0 & 57.6 & 7.1 & 10.3 & NA & 1.0 & 8.0 & NA & 1.1 & 78.3 & 12.0 & 9.4 & (Spranghers et al., 2017) \\
\hline $\begin{array}{l}\text { Cafeteria waste } \\
\quad(\text { Mean } \pm S D, n=3)\end{array}$ & Prepupae & NA & $44.9 \pm 1.5$ & $8.3 \pm 0.6$ & $13.5 \pm 0.7$ & $2.4 \pm 0.2$ & $2.1 \pm 0.2$ & $12.0 \pm 0.7$ & $9.9 \pm 0.5$ & $0.1 \pm 0.0$ & $69.9 \pm 1.4$ & $14.9 \pm 0.8$ & $12.5 \pm 0.4$ & (Surendra et al., 2016) \\
\hline $\begin{array}{l}\text { Vegetable and fruit waste } \\
(7: 3)\end{array}$ & Larvae & NA & 52.1 & 10.4 & 13.9 & NA & 2.6 & 8.5 & 7.0 & 1.7 & 78.9 & 12.3 & 8.8 & (Meneguz et al., 2018) \\
\hline $\begin{array}{l}\text { Vegetable and fruit waste } \\
\quad(17: 3)(\text { Mean } \pm S D \\
n=3)\end{array}$ & Larvae & 0.5 & 28.1 & 3.8 & 5.8 & 1.6 & 0.7 & 4.3 & 1.3 & 10.3 & 73.5 & 14.9 & 11.6 & (Giannetto et al., 2019) \\
\hline $\begin{array}{l}\text { Vegetable and fruit waste } \\
\quad(17: 3) \text { (Mean } \pm \text { SD, } \\
n=3)\end{array}$ & Prepupae & 1.2 & 61.9 & 9.1 & 7.9 & 2.4 & 1.1 & 5.3 & 2.4 & 4.7 & 84.5 & 8.4 & 7.1 & (Giannetto et al., 2019) \\
\hline Fruit waste & Larvae & NA & 57.4 & 9.6 & 13.1 & NA & 1.8 & 9.3 & 4.1 & 0.7 & 81.9 & 13.3 & 4.8 & (Meneguz et al., 2018) \\
\hline Winery by-product & Larvae & NA & 34.7 & 6.6 & 18.9 & NA & 2.8 & 12.5 & 17.6 & 0.4 & 63.0 & 19.0 & 18.0 & (Meneguz et al., 2018) \\
\hline Brewery by-product & Larvae & NA & 32.4 & 6.7 & 20.4 & NA & 1.8 & 9.2 & 23.6 & 2.5 & 61.3 & 12.7 & 26.0 & (Meneguz et al., 2018) \\
\hline Dairy manure & Larvae & 3.1 & 35.6 & 7.6 & 14.8 & 3.8 & 3.6 & 23.6 & 2.1 & NA & NA & NA & NA & (Li et al., 2011b) \\
\hline $\begin{array}{l}\text { Soybean curd } \\
\quad \text { residue + Bacteria } \\
\quad \text { (Lactobacillus buchneri } \\
\quad(\text { Mean } \pm \mathrm{SE}, \mathrm{n}=3 \text { ) }\end{array}$ & Larvae & $1.5 \pm 0.1$ & $39.6 \pm 1.5$ & $6.5 \pm 0.2$ & $13.1 \pm 0.5$ & $2.7 \pm 0.1$ & $2.1 \pm 0.1$ & $11.5 \pm 0.4$ & $17.5 \pm 0.4$ & $1.4 \pm 0.1$ & NA & NA & NA & (Somroo et al., 2019) \\
\hline $\begin{array}{l}\text { Soybean curd residue } \\
(\text { Mean } \pm \mathrm{SE}, \mathrm{n}=3)\end{array}$ & Larvae & $1.7 \pm 0.1$ & $36.9 \pm 1.2$ & $5.9 \pm 0.2$ & $13.2 \pm 0.4$ & $3.2 \pm 0.2$ & $1.7 \pm 0.1$ & $12.1 \pm 0.6$ & $17.0 \pm 0.5$ & $1.2 \pm 0.0$ & NA & NA & NA & (Somroo et al., 2019) \\
\hline $\begin{array}{l}\text { Brown algae (Ascophyllum } \\
\text { nodosum) (Mean } \pm \mathrm{SD} \text {, } \\
\mathrm{n}=4 \text { ) }\end{array}$ & Larvae & NA & $23.9 \pm 2.9$ & $6.7 \pm 0.5$ & $16.6 \pm 0.1$ & $2.5 \pm 0.1$ & $4.1 \pm 0.1$ & $17.9 \pm 1.1$ & $18.6 \pm 0.9$ & $1.6 \pm 0.1$ & $52.5 \pm 3.5$ & $22.2 \pm 1.3$ & $24.2 \pm 1.7$ & (Liland et al., 2017) \\
\hline Food waste & Prepupae & 1.4 & 41.1 & 0.4 & 12.2 & 3.2 & 2.4 & 14.1 & 13.8 & NA & NA & NA & NA & (Salomone et al., 2017) \\
\hline $\begin{array}{l}\text { Food waste (Mean } \pm \mathrm{SE} \text {, } \\
\quad \mathrm{n}=3 \text { ) }\end{array}$ & Prepupae & $2.3 \pm 0.2$ & $56.2 \pm 5.8$ & $9.3 \pm 0.8$ & $10.3 \pm 1.1$ & $2.4 \pm 0.2$ & $1.9 \pm 0.2$ & $7.1 \pm 0.7$ & $10.3 \pm 1.2$ & NA & NA & NA & NA & (Wang et al., 2020) \\
\hline $\begin{array}{l}\text { Pig manure (Mean } \pm S E \text {, } \\
\quad n=3)\end{array}$ & Prepupae & $2.1 \pm 0.2$ & $56.4 \pm 6.4$ & $8.5 \pm 0.7$ & $11.0 \pm 1.0$ & $2.2 \pm 0.2$ & $1.5 \pm 0.1$ & $7.1 \pm 0.7$ & $10.2 \pm 1.0$ & NA & NA & NA & NA & (Wang et al., 2020) \\
\hline $\begin{array}{l}\text { Chicken manure } \\
\quad(\text { Mean } \pm \mathrm{SE}, \mathrm{n}=3)\end{array}$ & Prepupae & $1.6 \pm 0.1$ & $56.9 \pm 4.8$ & $8.2 \pm 0.9$ & $8.4 \pm 0.8$ & $1.9 \pm 0.1$ & $1.4 \pm 0.1$ & $7.0 \pm 0.6$ & $10.7 \pm 1.0$ & NA & NA & NA & NA & (Wang et al., 2020) \\
\hline $\begin{array}{l}\text { Cow manure (Mean } \pm \mathrm{SE} \text {, } \\
\mathrm{n}=3 \text { ) }\end{array}$ & Prepupae & $1.7 \pm 0.1$ & $54.2 \pm 4.0$ & $7.0 \pm 0.7$ & $7.9 \pm 0.6$ & $2.1 \pm 0.2$ & $1.2 \pm 0.1$ & $6.3 \pm 0.6$ & $11.0 \pm 0.9$ & NA & NA & NA & NA & (Wang et al., 2020) \\
\hline $\begin{array}{l}\text { Fermented maize straw } \\
\quad(\text { mean } \pm \mathrm{SE}, \mathrm{n}=3)\end{array}$ & Prepupae & $0.8 \pm 0.0$ & $22.4 \pm 0.4$ & $4.4 \pm 0.2$ & $13.1 \pm 0.3$ & $1.2 \pm 0.1$ & $1.9 \pm 0.1$ & $23.3 \pm 0.5$ & $24.0 \pm 0.3$ & $1.3 \pm 0.1$ & $45.4 \pm 1.1$ & $24.9 \pm 0.6$ & $25.4 \pm 0.3$ & (Gao et al., 2019) \\
\hline
\end{tabular}




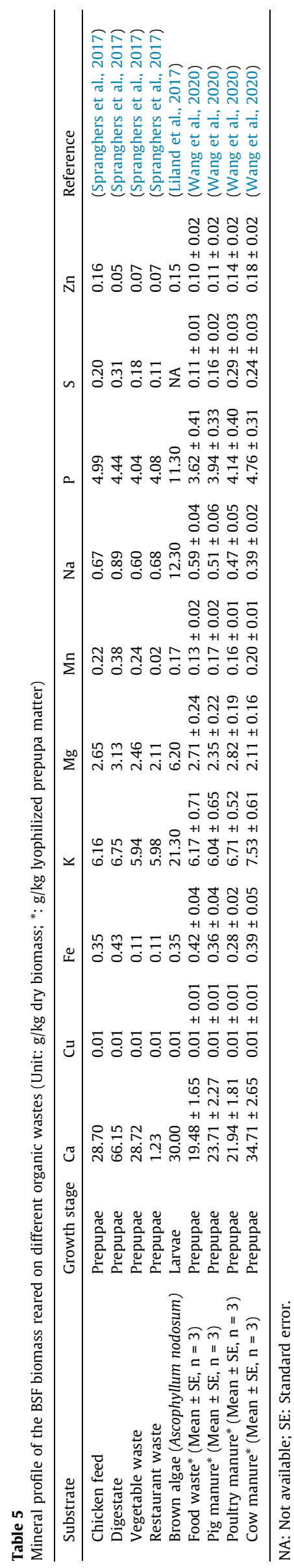

Chitin is another important component of BSF biomass ( $\sim 7 \%$ of dry matter), and is often falsely counted as crude protein in BSF biomass because of nitrogen present in the chitin molecule; the crude protein content is usually determined by multiplying the total Kjeldahl nitrogen value by a factor of 6.25 (Finke, 2007; Janssen et al., 2017). Despite studies have reported that a high content of chitin and chitin derivative chitosan in the diet negatively affects nutrient utilization (Karlsen et al., 2017; Khempaka et al., 2011; Kroeckel et al., 2012; Marono et al., 2016; Razdan and Pettersson, 1994; Schiavone et al., 2017; Vidanarachchi et al., 2010), these compounds have been reported to have antimicrobial and prebiotic properties (Komi et al., 2018), which are discussed in section 6.1.

Nutrient composition of the BSF biomass, however, is highly dependent on the substrate fed to the larvae (Barroso et al., 2017; Danieli et al., 2019; Ewald et al., 2020; Gold et al., 2020; Lalander et al., 2019; Liland et al., 2017; Meneguz et al., 2018; Spranghers et al., 2017), larval growing conditions (Palma et al., 2019), processing methods (Huang et al., 2018; Larouche et al., 2019; Leni et al., 2019; Tschirner and Simon, 2015), and life stage at harvest (Giannetto et al., 2019; Kawasaki et al., 2019; Liu et al., 2017; Smets et al., 2020). For example, the carbohydrate content in the substrate influences the lipid content in the BSF larvae (Gold et al., 2018; Li et al., 2015). When BSF larvae are fed low protein and high carbohydrate substrates, carbohydrate is mainly converted by larvae into lipids (Pimentel et al., 2017). Several studies have reported higher lipid content in BSF larvae grown on the substrates low in protein and rich in carbohydrate when compared to larvae grown on substrates with balanced protein and carbohydrate (Barragán-Fonseca, 2018; Danieli et al., 2019; Gold et al., 2018; Jucker et al., 2017; Spranghers et al., 2017). While some feedstocks rich in protein improve protein content (in BSF biomass), larval weight, bioconversion and feed conversion rates, and reduce development time (Gold et al., 2018; Nguyen et al., 2013; Oonincx et al., 2015), the amino acid profile in BSF biomass is not greatly influenced by the amino acid profile of the substrate (Spranghers et al., 2017). The lipid profile of the substrate, however, directly influences the lipid profile of the BSF biomass (Ewald et al., 2020; Gold et al., 2018; Liland et al., 2017; Somroo et al., 2019).

Abiotic factors such as larval-rearing temperature also affect the nutrient profile of the BSF biomass. The crude protein content in BSF biomass, for example, decreased when the rearing temperature was increased from 28 to $34{ }^{\circ} \mathrm{C}$ (Palma et al., 2019). Similarly, processing method also affects the nutrient quality of the BSF biomass (Huang et al., 2018; Larouche et al., 2019; Leni et al., 2019). For example, compared to blanching, slow killing of BSF prepupae by freezing activates enzymatic reactions including melanization with tyrosine consumption, energetic metabolism, and lipolysis, thereby impacting the digestibility and nutritional quality (loss of cysteine and lysine) of BSF biomass (Leni et al., 2019). Moreover, blanching was found to result in low lipid oxidation, reduction in moisture content, color stability, and significantly reduced the microorganisms in the insect biomass (Larouche et al., 2019). Similarly, compared to microwave drying, BSF larvae protein prepared by conventional drying $\left(60{ }^{\circ} \mathrm{C}\right)$ had a significantly higher Digestible Indispensable Amino Acid Score (DIAAS) and better digestibility (Huang et al., 2018). A substantial increase in the crude protein and decrease in gross energy contents in BSF prepupae was reported after partial or complete removal of fat from BSF prepupae (Surendra et al., 2016).

In general, the protein derived from BSF biomass has a good balance of essential and non-essential amino acids (Table 3). Except for methionine and lysine, BSF biomass has both essential and non-essential amino acids comparable to that of fish meal (Belghit et al., 2019; Henry et al., 2015; Liland et al., 2017; 
Makkar et al., 2014; Surendra et al., 2016). The concentration of the amino acids such as methionine, lysine, tryptophan, threonine, cysteine, and valine, which are often limiting amino acids in poultry and pig diets, is comparable to soybean meal. Additionally, BSF larvae are a rich source of bioavailable arginine (Belghit et al., 2019), which is often a limiting amino acid in plant-based protein (Andersen et al., 2013).

Lauric (C12:0), palmitic (C16:0), and oleic (C18:1) acids are the major fatty acids present in the fat derived from BSF biomass, accounting for about $32-60 \%, 8-20 \%$, and $5-12 \%$ of total fatty acids, respectively (Table 4 ). Moreover, saturated fatty acids accounts for about $61-82 \%$ of total fatty acids, while monounsaturated and polyunsaturated fatty acids contribute to $9-19 \%$ and $4-26 \%$ of total fatty acids, respectively (Table 4). Although nutritionally important omega- 3 and omega- 6 fatty acids are present in BSF biomass, the concentrations of these polyunsaturated fatty acids are lower than in fish meal. However, as mentioned earlier, since amount and fatty acid profile of fat in the BSF biomass are directly related to the amount and type of fatty acids present in the substrate fed to BSF larvae, nutritionally important fatty acids, such as omega-3 and omega- 6 fatty acids, could be enriched in the BSF biomass by manipulating the composition of substrate fed to the BSF larvae. For example, studies have reported a substantial increase in omega-3 fatty acids content in the BSF larvae fed with diets containing microalgae (Truzzi et al., 2020), seaweed (Liland et al., 2017), and fish offal (St-Hilaire et al., 2007a).

Like other nutrients, the micronutrients present in the BSF biomass is also dependent on substrate fed. In general, BSF biomass accumulates calcium (Ca) and manganese (Mn), but not sodium (Na) or sulfur (S) (Spranghers et al., 2017). Thus, the Ca (the most abundant mineral in BSF biomass) content in BSF biomass (up to $6 \%$ of dry matter, Table 5 ) is comparatively higher than that in other insects and fish meal (Wang and Shelomi, 2017).

The variation in nutrient composition of BSF biomass is both an opportunity and a challenge for BSF-based animal feed applications. It provides an opportunity to tailor the nutrient profile of the BSF biomass by manipulating the composition of the substrates fed to BSF larvae, harvesting the BSF biomass at appropriate stage (larval, prepupal, pupal), and processing of the harvested biomass. Similarly, since the characteristics of substrates vary substantially, maintaining consistency in the nutrient profile of the BSF biomass, especially when BSF system is employed for treating diverse organic wastes, could be challenging.

\section{Black soldier fly biomass as animal/aquatic feed}

BSF biomass has been studied as a protein-rich alternative feed for several decades. Although studies have reported mixed results on performance of BSF meal (larval-, prepupal-, or pupal-meal) as an animal feed depending on factors such as animal types, feed formulations, and feeding duration, several studies have shown that BSF meal could substitute a significant amount of conventional sources of protein (e.g., soybean meal and fish meal) in animal diets. The performances of BSF meal as poultry, fish, and swine feed are critically examined in the following sections.

\subsection{Poultry}

BSF meal has been widely studied as a poultry feed (Borrelli et al., 2017; Brede et al., 2018; Cullere et al., 2019, 2016; Cutrignelli et al., 2018; Dabbou et al., 2018; Hale, 1973; Kawasaki et al., 2019; Kierończyk et al., 2019; Lee et al., 2018; Marono et al., 2017; Maurer et al., 2016; Mbhele et al., 2019; Mwaniki et al., 2018, 2019; Nery et al., 2018; Onsongo et al., 2018; Pieterse et al., 2019; Ruhnke et al., 2018; Schiavone et al.,
2016, 2018, 2019; Secci et al., 2018, 2020; Veldkamp and van Niekerk, 2019; Wallace et al., 2018, 2017; Zotte et al., 2019). Studies have shown mixed results on performance of BSF meal as a poultry feed depending on poultry type (e.g., broiler or layer), poultry growth stage (e.g., starter, growing, or finishing stage), feeding duration, feeding mode (e.g., caged-fed or free range), inclusion rate of BSF meal in the diet, BSF meal types (depending on feedstocks used for growing BSF larvae), and method of processing the BSF larvae (e.g., dried or fresh, whole, chopped, or milled, defatted or non-defatted).

For example, no significant difference was observed between the treatment and the control diets in terms of diet preference, apparent digestibility of major nutrients, productive performance, mortality, carcass traits, and sensory and meat quality characteristics while feeding chicken; Cobb 500 (Pieterse et al., 2019) and Ardennaise (Moula et al., 2018), and quail (Coturnix coturnix japonica) (Cullere et al., 2016) with diet containing up to $15 \%$ of BSF biomass. However, Dabbou et al. (2018) observed mixed results while partially defatted BSF larval meal was used as a soybean meal substitute (up to 15\%) in the diets of broiler chickens (Ross 308). The diet containing BSF larval meal improved live weight and daily feed intake of chickens during the starter period (1-10 days), but negatively affected the feed conversion ratio during both the growing (10-24 days) and finishing (24-35 days) periods.

Similar to broiler chickens, the effects of including BSF meal in the diets of layers have been inconsistent (Cutrignelli et al., 2018; Marono et al., 2017; Maurer et al., 2016; Ruhnke et al., 2018). In general, replacement (up to $100 \%$ ) of soybean meal with BSF meal for a short period (up to 6 weeks) did not affect the performance (e.g., body weight, feed intake, feed conversion ratio, laying percentage, and egg quality) of laying hens (Maurer et al., 2016; Ruhnke et al., 2018). However, feeding BSF meal or larvae as a soybean meal substitute for a longer period (over 12 weeks) negatively affected the performance (Cutrignelli et al., 2018; Marono et al., 2017; Ruhnke et al., 2018). The reduced performance of the hens fed with BSF meal was attributed to either reduced availability of the heat labile amino acids (i.e., lysine, arginine, threonine) due to the Maillard reaction because the BSF larvae were dried at $90{ }^{\circ} \mathrm{C}$ for 60 min prior to grinding (Ruhnke et al., 2018), lower protein digestibility due to presence of chitin in BSF meal (Cutrignelli et al., 2018), or to lower feed intake possibly due to the darker color of BSF meal than soybean meal (Cutrignelli et al., 2018; Marono et al., 2017). Blackening of the BSF meal is due to the formation of iron-polyphenol complexes upon grinding BSF larvae (Janssen et al., 2019).

The mixed results observed in different studies could partly be due to differences in nutrient quality of the BSF biomass used. In addition, since the nutrient requirement of poultry varies with growth stage and poultry type, such variation may have also contributed to inconsistency on the performance of BSF meal as a poultry feed. Inconsistencies among the results of different feeding trials are partly due to differences in experiments, as the diets (i.e., control and experimental) fed to poultry were not isoenergetic and isoproteic/isonitrogenous in all studies. In general, studies have shown that BSF meal could substitute a substantial portion of the soybean meal in poultry diets without negatively impacting, if not improving, the performance and quality of products.

\subsection{Fish}

BSF meal as fish feed has been tested for different species of fish for about four decades. Several studies have investigated different aspects (including nutrient digestibility, growth performance, and meat quality) of including BSF meal as a substitute for fish and soybean meal in the diets of fish species including channel catfish (Ictalurus punctatus) (Bondari and Sheppard, 1987), yellow catfish 
(Pelteobagrus fulvidraco) (Hu et al., 2017; Xiao et al., 2018a), African catfish (Clarias gariepinus) (Fawole et al., 2020), Nile tilapia (Oreochromis niloticus) (Devic et al., 2018; Muin et al., 2017; Toriz-Roldan et al., 2019), blue tilapia (Oreochromis aureus) (Bondari and Sheppard, 1987), rainbow trout (Oncorhynchus mykiss) (Borgogno et al., 2017; Bruni et al., 2018; Cardinaletti et al., 2019; Dumas et al., 2018; Huyben et al., 2019; Mancini et al., 2018; Renna et al., 2017; Rimoldi et al., 2019; Rumpold et al., 2018; Sealey et al., 2011; Secci et al., 2019; Stadtlander et al., 2017; St-Hilaire et al., 2007b; Terova et al., 2019), turbot (Psetta maxima) (Kroeckel et al., 2012), European seabass (Dicentrarchus labrax) (Magalhães et al., 2017), Japanese seabass (Lateolabrax japonicus) (Wang et al., 2019), Atlantic salmon (Salmo salar) (Belghit et al., 2018, 2019a, 2019b; Biancarosa et al., 2019; Bruni et al., 2020; Lock et al., 2016; Stenberg et al., 2019), zebrafish (Danio rerio) (Zarantoniello et al., 2019), Siberian sturgeon (Acipenser baerii) (Caimi et al., 2020), Jian carp (Cyprinus carpio var. Jian) (Li et al., 2016, 2017; Zhou et al., 2018), meagre (Argyrosomus regius) (Guerreiro et al., 2020), and climbing perch (Anabas testudineus) (Vongvichith et al., 2020).

Studies have shown that BSF meal could substitute up to $50 \%$ of fish meal in the diets of rainbow trout without significantly affecting survival, growth performance, condition factor (body weightto-body length ratio), somatic indices, and yield and physical quality of fillet (Bruni et al., 2018; Mancini et al., 2018; Renna et al., 2017). More importantly, compared to the fish fed control diets, higher microbial diversity was observed in the intestine of the fish fed diets containing BSF meal, which could contribute to immunity, feed digestibility, physiological function, and welfare of the fish (Bruni et al., 2018). However, a higher inclusion rate of BSF meal (i.e., 50\% replacement of fish meal) increased saturated fatty acids, mainly lauric acid, and decreased the mono- and poly-unsaturated (omega-3 and omega-6) fatty acids content in the fillet, thereby worsening the lipid health indices of the muscle (Mancini et al., 2018; Renna et al., 2017). On the contrary, St-Hilaire et al. (2007b) reported an increase in moisture and decrease in lipid and gross energy contents in rainbow trout (whole-body proximate composition) fed diets containing BSF prepupal meal as fish meal substitute; these differences could have arisen from the diet formulation, as tested diets were non-isoproteic and non-isolipidic.

Similarly, the use of BSF meal as substitute for fish and/or soybean meal in the diets of freshwater and marine Atlantic salmon had no significant effects on feed intake, feed conversion ratio, or whole-body contents of protein, lipid, amino acids, and minerals (Belghit et al., 2018, 2019).

The lack of a difference in growth of rainbow trout and Atlantic salmon fed with diets containing BSF meal could be due to the external supplementation of methionine and lysine in the diets containing BSF meal to meet the dietary requirements of the respective fishes (Belghit et al., 2018, 2019; Dumas et al., 2018; Lock et al., 2016). However, fish species differ in their amino acid requirements and are likely to tolerate different levels of fish meal substitution without supplementation of essential amino acids, despite low levels of lysine in the BSF-based diet (Belghit et al., 2019). Growth performance of European seabass was not affected when fish meal was substituted with BSF meal (inclusion rate of 19.5\%) without supplementation of amino acids (Magalhães et al., 2017).

Studies have reported mixed results regarding the effects of feeding BSF meal on product quality (slaughter quality) depending on fish type, inclusion level, BSF meal type (enriched vs nonenriched), and supplementation of amino acids (Belghit et al., 2019; Mancini et al., 2018; Renna et al., 2017). For example, Renna et al. (2017) found increased dry matter and ether extract contents in the dorsal fillets of rainbow trout fed diet containing
40\% BSF larval meal compared to the fish fed diets containing $20 \%$ or $0 \%$ BSF meal. However, other studies observed no significant effect of dietary inclusion of BSF meal on whole body composition of carp and Atlantic salmon (Belghit et al., 2019). However, studies have reported effects on the fatty acid profile of the fish fed diets containing BSF meal (Belghit et al., 2019; Mancini et al., 2018). Such variation in the fatty acid profile is likely to impact the sensory attributes (aroma and flavor) of fish products (Borgogno et al., 2017). However, findings on the dietary effects of BSF meal on sensory parameters of fish products are inconsistent (Belghit et al., 2019; Borgogno et al., 2017; Lock et al., 2016; Sealey et al., 2011).

Overall, studies have reported successful inclusion of BSF meal (up to $40 \%$ ) as a fish meal substitute in the diets of different species of fish without significantly affecting the performance and quality of products, thereby indicating the potential of BSF meal as an alternative protein source for different species of fish. However, higher chitin content (negatively affects digestibility of the nutrients) and lack of proper balance of limiting amino acids (especially methionine and lysine) and fatty acids (especially omega- 3 and omega- 6 fatty acids) could be the limiting factor for the higher inclusion of BSF meal in fish diet. Removing chitin from insect biomass before processing as fish feed or incorporating chitindigesting enzymes (chitinases) in fish diet could improve the digestibility of the fish feed containing higher proportion of BSF meal. Additionally, improving the nutrient profile of the BSF biomass, especially with regard to limiting amino acids (e.g., methionine and lysine) and fatty acids (e.g., omega-3 and omega- 6 fatty acids) contents by enriching the BSF larvae with substrate such as fish and/or algae processing wastes could allow for higher inclusion rates of BSF meal in diets for some fish species.

\subsection{Swine}

Compared to poultry and fish, BSF meal as a diet for swine has been less studied. Moreover, there is lack of consistency in performance of BSF meal fed pigs depending on the inclusion rate, feeding duration, and growth stage of the animal (Altmann et al., 2019; Biasato et al., 2019; Chia et al., 2019; Nekrasov et al., 2015, 2018; Newton et al., 1977; Spranghers et al., 2018; Velten et al., 2017; Yu et al., 2019a). Feeding piglets with diets containing BSF meal (inclusion rate up to $10 \%$, substitution of soybean meal up to $65 \%$ ) had no significant effect on growth performance, feed intake, feed-to-gain ratio, nutrient digestibility, gut morphology, and histological features (Biasato et al., 2019; Spranghers et al., 2018). However, a higher inclusion level of BSF meal in piglet diets (33\% inclusion rate or $75 \%$ substitution of soybean meal) resulted in negative effect on dry matter digestibility, feed intake, growth rate, and feed and protein conversion ratios (Newton et al., 1977; Velten et al., 2017).

There are limited studies examining the effects of feeding the diet containing BSF meal on carcass yield and quality in pigs. Although Yu et al. (2019a) reported improvement in average carcass trait and muscle chemical composition (when fed a diet containing 4\% BSF meal), Altmann et al. (2019) reported no significant effect on meat quality parameters, including sensory attributes (except stronger overall odors and higher juiciness values) when BSF meal was used as a soybean meal substitute. Yu et al. (2019a) attributed the improvement in meat quality to upregulation of lipogenic genes and genes related to muscle fiber composition. The contradictory observations between the studies could be attributed to the inclusion rate of BSF meal in the pig diet, feeding duration, and growth stage of the pig.

Overall, substantial amount of soybean meal could be replaced with BSF meal in feeding pigs without negatively affecting the 
growth performance and meat quality. However, extensive studies are necessary to determine the upper inclusion level of BSF meal, and its effects on the carcass yield and quality including sensory attributes.

\section{Nutraceutical value of the black soldier fly biomass as animal feed}

Besides their nutritional value, BSF meal is rich in bioactive compounds including chitin, medium chain fatty acids (C6-12), and antimicrobial peptides. A recent study identified a novel polysaccharide from BSF larvae (dipterose-BSF) capable of activating the innate immunity of mammalian macrophages (Ali et al., 2019). Thus, the possible antimicrobial and prebiotic effects of chitin and chitin derivatives, lauric acid, antimicrobial peptides, and novel polysaccharide present in BSF meal indicate that BSF meal not only provides an alternative to conventional sources of protein and energy in animal diet, but also a valuable alternative to in-feed antibiotics and prebiotics, thereby adding value to BSF meal in the diets of monogastrics. Additionally, inclusion of BSF meal may further increase the shelf-life of the feeds (Henry et al., 2015; Zhao et al., 2010). The potential benefits of chitin and chitin derivatives, lauric acid, and antimicrobial peptides present in BSF on animal health are discussed in the following section.

\subsection{Chitin}

Chitin and chitosan have a stimulatory effect on innate immune system (Lee et al., 2008), antimicrobial properties, and bacteriostatic effect on diverse, harmful, gram-negative bacteria, and positive effects on growth of beneficial microbes (Karlsen et al., 2017; Zhou et al., 2013). Although BSF meal, which contains $6-7 \%$ chitin on dry matter basis, has not been extensively studied as a source of chitin and chitosan, or for its prebiotic or stimulatory effects on the immune system, supplementing chitin in the diets of broilers and fish has shown positive effects (e.g., inhibition of pathogens and enhancement of beneficial intestinal microbiota) (Karlsen et al., 2017; Khempaka et al., 2011). For example, positive modification of gut microbiota of rainbow trout fed diets containing BSF meal (increased occurrence of well-known probiotics, Carnobacterium spp.) was attributed to the presence of fermentable chitin in the BSF meal (Bruni et al., 2018; Rimoldi et al., 2019; Terova et al., 2019). Chitin has also been suggested to have positive effects on prolidase activity, an enzyme responsible for hydrolysis and adsorption of proline and hydroxyproline in the small intestine of rainbow trout (Dumas et al., 2018). Moreover, acidic chitinase present in the stomach and intestine of chickens is capable of degrading chitin to chitin-oligosaccharides (Tabata et al., 2017). Feeding the diet containing BSF meal to laying hens significantly modified the gut microbiota composition (increase in population of potential chitin degraders) and increased short chain fatty acid production, suggesting a potential prebiotic effect of BSF meal (Borrelli et al., 2017). Thus, the inclusion of chitin-rich BSF meal in animal feed could increase the shelf-life of feed and reduce the use of antibiotics and prebiotics in animal production (Rimoldi et al., 2019; Yu et al., 2019b). Additionally, since neither fish, birds, nor mammals are capable of synthesizing chitin (Gasco et al., 2018), incorporating BSF biomass (as a source of chitin) in the diets of these animals could potentially stimulate their immune system.

\subsection{Lauric acid}

As pointed out earlier, BSF biomass is rich in medium chain fatty acids, particularly lauric acid (30-60\% of total fatty acids) (Table 4). Like commonly-used antimicrobial agents, medium chain fatty acids have antimicrobial effects on a wide variety of pathogens, including Mycobacterium smegmatis, Chlamydia trachomatis, Listeria monocytogenes, Staphylococcus aureus, Neisseria gonorrhoeae, and Helicobacter pylori (Churchward et al., 2018; Decuypere and Dierick, 2003; Gasco et al., 2018). Moreover, the fat from BSF prepupae has substantial antimicrobial effects against lactobacilli and D-streptococci (Spranghers et al., 2018). More importantly, these fatty acids as antimicrobial agents neither have a significant human or animal toxicity nor induce problem of residues and cross-resistance induction (Decuypere and Dierick, 2003). Thus, the BSF meal rich in medium chain fatty acids, especially lauric acid, could be a valuable alternative not only to conventional protein and energy source in animal diets but also to in-feed antibiotics used for promoting growth, and preventing and curating gastrointestinal diseases (Decuypere and Dierick, 2003).

\subsection{Antimicrobial peptides}

Insects produce a variety of antimicrobial peptides (Tonk and Vilcinskas, 2017; Vogel et al., 2018; Yi et al., 2014), which have effects against several types of bacteria, in which risk of bacterial resistance is low (Chernysh et al., 2015). BSF genome encodes 50 antimicrobial peptides, the largest antimicrobial peptide family identified in any insects (Zhan et al., 2020). Furthermore, studies have reported the strong negative effects of antimicrobial peptides derived from BSF larvae on gram-negative bacteria (Choi et al., 2018; Park and Yoe, 2017) and gram-positive bacteria (Park et al., 2015). Although the effects of antimicrobial peptides present in BSF biomass on the performance of different animals and/or against pathogenic agents are not well studied, a substantial decrease in diarrhea in the weaned piglets fed with diet containing insect meal (e.g., yellow mealworm, giant mealworm, house fly larval meal) was ascribed to the antimicrobial peptides present in the insect meal (Ji et al., 2016). With growing global concern of increasing antibiotics resistant bacteria and genes in the environment, antimicrobial peptides present in insect biomass are of significant interest, especially such antimicrobial peptides could potentially act against fungi and viruses (Gasco et al., 2018).

\section{Microbial and chemical safety of the black soldier fly biomass as animal feed}

Organic wastes are often contaminated with diverse microorganisms and pharmaceuticals (human feces and animal manures), pesticides (fruit and vegetable wastes), mycotoxins (improperly stored milling and brewery side streams), heavy metals and other toxins including dioxins, polychlorinated biphenyls and polyaromatic hydrocarbons (from organic fraction of municipal solid waste) (Gold et al., 2018). Thus, the presence of microbial and chemical contaminants in BSF biomass needs to be closely monitored to ensure the feed safety, especially if the organic wastes contaminated with such contaminants are used as substrate in rearing BSF larvae (Purschke et al., 2017). However, compared to the nutritional composition, the risk (e.g., microbial and chemical contaminations) associated with the use of the BSF for bioconversion of organic wastes into animal feed has not been well studied, thereby limiting the use of the BSF biomass reared on a variety of organic wastes to animal feeds (Gold et al., 2018).

Though BSF treatment is reported to reduce pathogenic microbes such as Salmonella spp. (Erickson et al., 2004; Lalander et al., 2015) and Enterococcus coli (Erickson et al., 2004; Liu et al., 2008) present in the organic wastes such as human feces and animal manures, studies have reported presence of food pathogens such as Salmonella spp. and Bacillus cereus both in BSF biomass and residues (Wynants et al., 2019). Moreover, treating the waste with BSF larvae had no effects on oocysts or eggs of the parasites such as Eimeria tenella, E. nieschulzi, and Ascaris suum (Muller 
et al., 2019). Thus, proper pretreatment is necessary before using BSF biomass as animal feed and residue as fertilizer, especially when applied for growing food crops.

The gut microbiota of insects is fairly resistant to mild treatments such as heat (harsh treatments would negatively affect the nutrient quality of the insect biomass). Killing BSF larvae or prepupae by blanching could be one option as it not only reduces the microbial load in the BSF biomass, but is also rapid and less costly, mitigates lipid oxidation, increases color stability, and enhances flavor and taste (Larouche et al., 2019). Additionally, since studies have reported differences in core microbiota in larval gut (Shelomi et al., 2020; Wynants et al., 2019; Zheng et al., 2013), both nutritional and microbial composition of the substrate are likely to influence the larval gut microbiota (Bruno et al., 2019; De Smet et al., 2018; Jeon et al., 2011). Thus, the gut microbiota of BSF could be positively modified via dietary supplementation (Rumpold and Schlüter, 2013).

Possible bioaccumulation of heavy metals, pesticides, and pharmaceuticals is of serious concern while rearing BSF on the diverse organic wastes contaminated with such chemicals. For example, when BSF larvae were reared on the experimental substrate spiked with heavy metals, $\mathrm{Cd}$ and $\mathrm{Pb}$ bioaccumulated in the BSF biomass, with the bioaccumulation factor (concentration in the organism divided by the concentration in the substrate) of 6.1-9.5 and 1.2-2.6, respectively, exceeding the statutory thresholds for animal feed (Purschke et al., 2017; Tschirner and Simon, 2015; van der Fels-Klerx et al., 2016b), while no bioaccumulation was observed for chromium ( $\mathrm{Cr})$, nickel $(\mathrm{Ni})$, As, zinc $(\mathrm{Zn})$, and mercury $(\mathrm{Hg})$ (Diener et al., 2015; Purschke et al., 2017). Similarly, no bioaccumulation of mycotoxins (aflatoxins B1/B2/G2, deoxynivalenol, ochratoxin A, zearalenone) (Bosch et al., 2017; Camenzuli et al., 2018; Gulsunoglu et al., 2019; Lalander et al., 2016; Meijer et al., 2019; Purschke et al., 2017), pesticides (chlorpyrifos, chlorpyrifos-methyl, pirimiphos-methyl, azoxystrobin, propiconazole) (Bosch et al., 2017; Gulsunoglu et al., 2019; Lalander et al., 2016; Purschke et al., 2017), and pharmaceuticals (carbamazepine, roxithromycin, trimethoprim) (Lalander et al., 2016) have been observed in BSF biomass. The concentration of pesticides and pharmaceuticals decreased in the residual substrate, which was attributed to degradation of these compounds by microorganisms associated with BSF larvae. Hence, the crops contaminated with compounds such as mycotoxins could be biosanitized using BSF larvae, providing an outlet for valuable crops that would otherwise have to be destroyed when mycotoxin concentration exceeds the legal safety levels for animal feed application. Contrary to Purschke et al. (2017), Biancarosa et al. (2018) found accumulation of As along with Cd in the BSF larvae fed with seaweed (Ascophyllum nodosum)-enriched media. Since Cd is believed to be transported into the cells via $\mathrm{Ca}^{2+}$ channels (Martín-Folgar and Martínez-Guitarte, 2017), a substantial bioaccumulation of Cd in BSF biomass could be attributed to the high concentration of $\mathrm{Ca}$ in BSF biomass (Finke, 2013; Somroo et al., 2019). Thus, while rearing BSF larvae for animal feed application on the organic wastes rich in heavy metals, especially $\mathrm{Cd}, \mathrm{Pb}$, and $\mathrm{As}$, one option for achieving product safety would be co-feeding of such organic wastes with the feedstocks which are fairly low in heavy metals. Additionally, when BSF technology is employed for treating organic wastes highly contaminated with such heavy metals which prohibits the feed application of BSF biomass, BSF protein and fat could be used for other applications including bioplastic, biodiesel, and biobased lubricant production.

\section{Environmental aspects of bioconversion of organic wastes into black soldier fly biomass}

Recently, several studies focused on the environmental impacts of bioconversion of organic wastes into BSF biomass. Most of these studies are based on data from small-scale bioconversion facilities (Bosch et al., 2019; Mertenat et al., 2019; Roffeis et al., 2020; Salomone et al., 2017; Smetana et al., 2016) and only few are from large-scale facilities (Smetana et al., 2019). The environmental impact of bioconversion of organic wastes into BSF biomass is not consistent across the different scales of production (data from small-scale or pilot-scale studies) and modes of life cycle assessment (LCA) (Attributional LCA or Consequential LCA). However, there is consensus among the studies that environmental impact is largely dependent on the types of organic wastes used in rearing the BSF larvae. In general, the environmental impact of producing BSF biomass is higher when the organic wastes which are already being used for food or feed applications are used to rear the BSF larvae, rather than rearing the larvae on animal manure and food waste (Bosch et al., 2019). For example, when organic wastes with food or feed value were used as substrate for producing BSF biomass, the environmental impacts (e.g., global warming potential, land use, and energy use) of BSF biomass were higher than that of soybean meal and fish meal. However, except for global warming potential, environmental impacts of rearing BSF larvae on animal manure and food waste were lower than that of fish meal and soybean meal (Bosch et al., 2019; Roffeis et al., 2020). But current feed safety regulations prohibit feeding farmed insects (which includes BSF as defined by the European Union) with organic wastes such as animal manure and catering wastes for food and feed applications in the European Union (European Commission, 2001, 2017; IPIFF, 2019). Additionally, studies have shown that energy input for processing, especially drying of BSF biomass is the major contributor to environmental impacts of BSF production (Feng et al., 2019; Smetana et al., 2019). Thus, improvement in efficiency of BSF biomass processing, which is most likely with the upscaling of the technology since most of the data for LCA were derived from the small-scale studies, or use of renewable sources of energy such as solar dryer and photovoltaic, the environmental impacts of the BSF biomass production is likely to improve.

Additionally, excessive fishing from natural water bodies for both food and feed applications has significant environmental issues, including the loss of biodiversity. Thus, although BSF meal as animal feed compared poorly in terms of land, water, and energy use as compared with fish meal, BSF meal may outperform fish meal in terms of other environmental and ecological impacts (Smetana et al., 2019). Furthermore, since studies have shown degradation of pesticides and pharmaceuticals while treating organic wastes using BSF larvae (Bosch et al., 2017; Lalander et al., 2016; Purschke et al., 2017), treating organic wastes with BSF larvae could minimize the spread of pharmaceuticals and pesticides into the environment (Lalander et al., 2016). Thus, environmental impact analyses should also account these aspects of bioremediation of the organic micro-pollutants by BSF biomass. Also, studies have shown that bioconversion of organic waste (e.g., segregated kitchen waste) into BSF biomass has lower direct GHGs emissions than composting (Mertenat et al., 2019). Thus, LCAs and techno-economic analysis (TEA) should also be conducted to compare the currently adopted technologies for treating organic wastes such as composting, direct feeding to animals, and anaerobic digestion with BSF farming for recovering resources from diverse organic wastes. 


\section{Economics of bioconversion of organic wastes into black soldier fly biomass}

Currently, studies on the economics of bioconversion of organic wastes into BSF biomass for animal feed application are limited. Moreover, although many private companies such as Agriprotein (South Africa), EnviroFlight (USA), Bioflytech (Spain), Enterra Feed Corporation (Canada), Entobel (Vietnam), Entofood (Malaysia), Entomo Farm (France), Hexafly (Ireland), F4F (Chile), Hermetia GmbH (Germany), InnovaFeed (France), and Protix (The Netherlands) are involved in the BSF larvae production business, information on their operational processes and financial aspects (e.g., costs and benefits) are not publicly disclosed; possibly to maintain their competitive advantages (Joly and Nikiema, 2019; Zurbrügg et al., 2018).

Joly and Nikiema (2019) have summarized the economic and environmental performances of BSF technology, which indicate that the economic performance of BSF technology varies substantially with location of the operation, substrates used, production scale, and intended use of the products. Some studies on the economic performance of BSF biomass production for animal feed have shown that the use of current technology (mostly dominated by batch mode operation using flat tray system, which are not only labor intensive but also require substantial space), has a higher cost than conventional animal feed such as fish meal and soybean meal (Caruso et al., 2013b; Pleissner and Smetana, 2019). For example, for a facility converting 53.6 tons of wet food waste into 3.64 tons of dried prepupae and 6.35 tons of dried fertilizer per day, the operation alone will cost $\$ 5,850(5,282 €)$ per day [i.e., about $\$ 1,600(1,451 €)$ per ton of dried prepupae] (Pleissner and Smetana, 2019). But, as a rule of thumb, for a profitable operation of organic waste to BSF biomass conversion facility, the production cost per ton of BSF biomass should not exceed $\$ 907$ ( $\$ 1,000$ per metric ton of BSF biomass) (Drew and Pieterse, 2015).

Life cycle cost analysis based on small-scale production has shown that labor accounts for $30-65 \%$ of the total operating cost (Joly and Nikiema, 2019), and labor and substrate acquisition account for about $90 \%$ of total cost of BSF-derived animal feed production (Roffeis et al., 2018). However, substrates, such as animal manure, food wastes, and sewage sludge, have negative values as the generators of such wastes must pay discharge fee (i.e., tipping fees) (Badgett et al., 2019; Yazan et al., 2018). Additionally, national, and/or local governments may provide incentives (including fiscal) for minimizing waste generation and/or managing wastes properly (Matheson, 2019). Thus, substantial improvement in economics of BSF-based animal feed production are likely to come from onsite production of BSF biomass using negativevalue wastes, with mechanization and process control, scaling up of the production systems, and with incentive-based governments' policies. For example, modelling and verification of business plans from insect factories have suggested that to be profitable, the bioconversion facility has to reach a processing scale of converting approximately 110 tons (100 metric tons) a day of organic waste into 7.72 tons (7 metric tons) of insect meal (Drew and Pieterse, 2015). However, the availability and use of under-utilized organic resources for the production of the BSF biomass would be critical for further development of the insect-based feed industry (Smetana et al., 2019). Moreover, BSF meal is rich in lauric acid, chitin, and antimicrobial peptides, which are reported to have antimicrobial and prebiotic attributes. Thus, if BSF meal could successfully substitute the use of antibiotics, prebiotics, and the conventional feedstuffs which are not certified as organic products, the animal and fish products could be certified as organic product and sold at a premium price thereby improving the economics of BSF meal as animal and fish feed.

\section{Challenges of bioconversion of organic wastes into black soldier fly biomass}

Compared to composting and anaerobic digestion, insect farming on organic wastes for resource recovery is relatively new technology. Most studies in this field have been conducted at a laboratory/bench-scale. Such an approach does not produce reliable data for industrial-scale application as bench-scale results do not always scale up in a linear fashion (Miranda et al., 2019). Two primary hurdles preventing the expansion to industrial scale are; (i) proprietary information related to methods employed by companies that prevent the use of said methods by others and (ii) lack of readily available infrastructure/add-on technology to explore such efforts. In both instances, one can be used to solve the other; industry could partner with academia to conduct such research without revealing trade secrets. Recently, such partnerships have begun to address the issues of scalability of benchscale to industrial-scale. One example is a pilot-scale study looking at short-, middle-, and long-term developments (consider it the progression of the insect agriculture industry) using sensitivity analyses (Smetana et al, 2019).

Regulations currently limit the expansion and diversification of insects in the food and feed sectors. For instance, regulations in the European Union limit the production of BSF to vegetable-based pre-consumer food waste (including dairy and eggs) and resulting products can only be used as feed for fur-producing animals, pets, and aqua-cultured fish (European Commission, 2017). Similar regulations exist in the United States (McDougal, 2018) and Canada (GlobeNewswire, 2018); however, in North America, BSF biomass can be used as poultry feed as well. The European Commission is currently exploring the possibilities for proposing a new revision of the feed ban rules in order to authorize pig and insect proteins in poultry feed when reared on substrates of vegetative origin (IPIFF, 2020). These regulations are in stark contrast with most of the world (e.g., China and other Asian countries), where BSF biomass can be produced on a variety of organic wastes and used as feed for a host of animals. However, the specific regulations in these nations are not transparent to the authors and require local regulatory expertise to interpret.

Concerning manure, studies have shown that BSF larvae can be reared on different types of manures. In many countries, significant amount of manure is produced than that can be assimilated/ applied in the available farmland, resulting in air, water, and soil pollutions (Ogejo, 2018). The question is whether the manure fed BSF biomass can be safely used as feed. If not, when the contamination level would be too high, biodegradation could be the purpose and the resulting larvae can still be used for non-feed purposes (van Huis, 2019), such as bioplastics, biolubricants, or biodiesel production.

A major challenge is to produce BSF meal that is competitively priced to that of fish meal and soybean meal. Avoiding loss, reuse, recycle, and landfills are the proposed measures for mitigating substantial amount of food currently being lost or wasted. Among the reuse methods, food waste is currently being investigated to be directly used as an animal feed which will make BSF biomass production on such substrate less competitive - economically and environmentally (Mohd-Noor et al., 2017; Pinotti et al., 2019). Thus, finding under-utilized and cheap organic waste streams would be a major challenge in mass production of BSF biomass. Another challenge could be making the organic waste stream high in carbon but low in nitrogen suitable for BSF larvae. Several options include the use of selffermentation as proposed for coconut endosperm (Mohd-Noor et al., 2017), pre-fermentation with fungi of corn stover (Gao 
et al., 2019), or adding nitrogen to almond hull-based feedstocks (Palma et al., 2019).

Several studies have shown that BSF larvae possess antimicrobial capability and are able to reduce pathogenic bacteria such as Salmonella spp. and Escherichia coli in the substrates (Wynants et al., 2019). So far, disease and pest problems have not been reported in the literature (Eilenberg et al., 2015). However, it cannot be ruled out the possible existence of disease and pest problems. Thus, disease and pests could pose potential risk in BSF farming on organic wastes especially when single strain of the BSF is used worldwide.

\section{Future perspectives}

The viability of the industry using BSF biomass as an animal feed will depend on whether it will be competitive to other feed sources such as fish meal and soybean meal, and/or if it, when further documented, can provide nutraceutical values. A reduced price could be achieved by using low-value waste streams (e.g., animal manure), process scale up, and automation with internet of things (IoT). A higher price for BSF biomass meal would be acceptable if there were additional benefits such as for health of humans or animals. Health promoting insect ingredients are chitin, fatty acids such as lauric acid, and proteins with antimicrobial properties such as phenoloxidases and enzymatic activities, such as amylase and trypsin (Muller et al., 2017; Nekrasov et al., 2018; Vogel et al., 2018). Besides, the use of insect ingredients in pharmaceuticals and cosmetics needs full attention (Rabani et al., 2019; Verheyen et al., 2018). Additionally, fatty acid profile of the BSF larval fat is very similar to palm kernel fat and coconut fat and could be an alternative to these fats for food applications (Matthäus et al., 2019). For example, a recent study has shown that BSF larval fat could replace up to $25 \%$ of butter in certain bakery products (Delicato et al., 2020), thereby indicating need for further investigation on possibility of using BSF biomass as food (Delicato et al., 2020; Wang and Shelomi, 2017).

Expansion of the waste streams used to produce BSF biomass could stabilize and expand the industry while offering greater protection to the environment. Resources such as post-consumer food wastes or animal manure are viable materials that can easily be used to produce BSF biomass. By allowing such resources to be used, the industry would be placed in a position where partnerships across industries (e.g., dairy, poultry, beef) or municipalities (e.g., urban areas producing large quantities of organic wastes) could be developed. Doing so would provide safe alternatives for recycling these organic resources while allowing appropriate levels of BSF biomass production. However, caution should be exercised while developing appropriate quality assurance plans to ensure the safety of the materials produced as a feed ingredient.

In addition to its applications as a fish meal and soybean meal substitute in the diets of livestock and aquatic animals, BSF biomass may find application as a substitute for high-price feed ingredients such as the bovine liver powder commonly used for mass rearing of mosquitos. The mass production of mosquitos is becoming more widespread due to its increased application in genetic control programs fighting against malaria and other diseases. However, the most commonly used feed ingredient, the bovine liver powder, is not only limited in supply but also is costly. A study examining the use of BSF larval meal as a feed for mass rearing two mosquito species, Aedes aegypti and A. albopictus, has shown that BSF larval meal could support the growth and development of mosquitos without negatively impacting the quality of produced mosquitos; rather there were improvements in several parameters (Mamai et al., 2019) thereby indicating the other possible applications of BSF biomass.
One less explored aspect is the use of BSF larvae in entomoremediation, which highlights the possibility of recovering metals such as Cd and Zn (Bulak et al., 2018). Nyakeri et al. (2019) also proposed bioremediation of fecal sludge from the environment using BSF. It has also been suggested to dispose of mycotoxincontaminated grain by using edible insects (Ochoa-Sanabria, 2019). Thus, in future, BSF technology may find wider applications in bioremediation of heavy metals, pesticides, and pharmaceuticals, and biosanitization of mycotoxins contaminated grains.

Studies on the genetic modification of BSF to improve their performance are currently limited. However, Zhan et al. (2020) have suggested that gene manipulation could generate mutant BSF lines with improved characteristics such as prolonged feeding duration consequently resulting in increased size BSF biomass. Furthermore, a recent study has provided insights on vital genes and regulators governing fat accumulation in BSF, thereby indicating potential of using genetic engineering approaches for manipulating BSF fat production for feed and fuel applications (Zhu et al., 2019).

With the advancement in breeding technology, there is possibility of exploiting the genetics of the BSF in improving both bioconversion efficiency and nutritional characteristics of BSF biomass (e.g., higher content of limiting essential amino acids and polyunsaturated fatty acids). Industries are collaborating with animalbreeding institutes to investigate the possibility of improving the BSF strains. An option is to select for strains that can handle certain types of waste streams. Another option is to exploit the strains that are present worldwide. A global population genetic survey of BSF based on polymorphic nuclear genetic markers compared four genetically distinct strains grown on three substrates (Sandrock et al., 2019). Both strains and substrate showed highly significant impacts on all life history traits and body compositional characteristics. The results imply that efficiency and sustainability of BSF production could be substantially increased by considering BSF genetics, and the interaction with the substrate.

The exponential increase in publications dealing with the BSF is spectacular; 384 journal papers were published in the last three years (2017-2019), compared to 137 papers published during the preceding 70 years (1946-2016) (Web of Science consulted on the 18 th of January 2020). This academic interest is a sign of the prospects of this insect in bioconversion. The BSF industry is likely be on the fore-front of the most promising technologies for recovering resources from diverse organic wastes. Studies have mostly focused on increasing the conversion efficiency and scaling up of the technology; however, more data are required for evaluating the economic and environmental performance of the BSF technology.

\section{Declaration of Competing Interest}

The authors declare that they have no known competing financial interests or personal relationships that could have appeared to influence the work reported in this paper.

\section{Acknowledgement}

This project was supported by funding from the Sun Grant Western Regional Center at Oregon State University (Corvallis, OR) and College of Tropical Agriculture and Human Resources at University of Hawai'i at Mānoa (Honolulu, HI).

\section{Author's contribution:}

K.C. Surendra: Formulated the content and scope of the paper, involved in interpretation of all information and prepared the first draft of the manuscript. 
Jeffery K. Tomberlin: Involved in writing background, BSF life cycle, bioconversion of organic wastes via BSF farming, bioconversion technologies, challenges of bioconversion of organic wastes into BSF biomass and future perspectives sections, and assisted in reviewing the manuscript.

Arnold van Huis: Involved in preparation of the challenges of bioconversion of organic wastes into BSF biomass and future perspectives sections, and assisted in collecting literature and reviewing the manuscript.

Jonathan A. Cammack: Involved in preparation of the BSF life cycle, bioconversion of organic wastes via BSF farming and bioconversion technologies sections, and assisted in reviewing the manuscript.

Lars-Henrik L. Heckmann: Involved in preparation of challenges of bioconversion of organic wastes into BSF biomass and future perspectives sections, and assisted in reviewing the manuscript.

Samir Kumar Khanal: Developed the initial idea, involved in planning the content of the paper and supervised the entire manuscript preparation as a principal investigator.

\section{References}

Abduh, M.Y., Jamilah, M., Istiandari, P., Manurung, S., Manurung, R., 2017. Bioconversion of rubber seeds to produce protein and oil-rich biomass using black soldier fly larva assisted by microbes. J. Entomol. Zool. Stud 5, 591-597.

Alexandratos, N., Bruinsma, J., 2012. World agriculture towards 2030/2050: The 2012 revision. ESA Working paper No. 12-03. Agricultural Development Economics Division. Food and Agriculture Organization of the United Nations, Rome.

Ali, M.F.Z., Ohta, T., Ido, A., Miura, C., Miura, T., 2019. The Dipterose of Black Soldier Fly (Hermetia illucens) Induces Innate Immune Response through Toll-Like Receptor Pathway in Mouse Macrophage RAW264.7 Cells. Biomolecules 9.

Alipour, N., Vinneras, B., Gouanve, F., Espuche, E., Hedenqvist, M.S., 2019. A ProteinBased Material from a New Approach Using Whole Defatted Larvae, and Its Interaction with Moisture. Polymers Basel, 11.

Altmann, B.A., Neumann, C., Rothstein, S., Liebert, F., Morlein, D., 2019. Do dietary soy alternatives lead to pork quality improvements or drawbacks? A look into micro-alga and insect protein in swine diets. Meat Sci 153, 26-34.

Andersen, S.M., Holen, E., Aksnes, A., Ronnestad, I., Zerrahn, J.E., Espe, M., 2013. Dietary arginine affects energy metabolism through polyamine turnover in juvenile Atlantic salmon (Salmo salar). Br J Nutr 110, 1968-1977.

Badgett, A., Newes, E., Milbrandt, A., 2019. Economic analysis of wet waste-toenergy resources in the United States. Energy 176, 224-234.

Banks, I.J., Gibson, W.T., Cameron, M.M., 2014. Growth rates of black soldier fly larvae fed on fresh human faeces and their implication for improving sanitation. Trop Med Int Health 19, 14-22.

Barbi, S., Messori, M., Manfredini, T., Pini, M., Montorsi, M., 2019. Rational design and characterization of bioplastics from Hermetia illucens prepupae proteins. Biopolymers 110, e23250.

Barragán-Fonseca, K.B., 2018. Flies are what they eat-Tailoring nutrition of Black Soldier Fly (Hermetia illucens (L.)) for larval biomass production and fitness. Wageningen University, p. 160.

Barroso, F.G., Sánchez-Muros, M.-J., Segura, M., Morote, E., Torres, A., Ramos, R., Guil, J.-L., 2017. Insects as food: Enrichment of larvae of Hermetia illucens with omega 3 fatty acids by means of dietary modifications. J. Food Compost. Anal. 62, 8-13.

Belghit, I., Liland, N.S., Gjesdal, P., Biancarosa, I., Menchetti, E., Li, Y., Waagbø, R., Krogdahl, Å., Lock, E.-J., 2019. Black soldier fly larvae meal can replace fish meal in diets of sea-water phase Atlantic salmon (Salmo salar). Aquaculture 503, 609619.

Belghit, I., Liland, N.S., Waagbø, R., Biancarosa, I., Pelusio, N., Li, Y., Krogdahl, Å., Lock, E.-J., 2018. Potential of insect-based diets for Atlantic salmon (Salmo salar). Aquaculture 491, 72-81.

Belghit, I., Waagbø, R., Lock, E.-J., Liland, N.S., 2019b. Insect-based diets high in lauric acid reduce liver lipids in freshwater Atlantic salmon. Aquacult. Nutr. 25, 343-357.

Bertinetti, C., Samayoa, A.C., Hwang, S.Y., 2019. Effects of Feeding Adults of Hermetia illucens (Diptera: Stratiomyidae) on Longevity, Oviposition, and Egg Hatchability: Insights Into Optimizing Egg Production. J Insect Sci 19.

Beskin, K.V., Holcomb, C.D., Cammack, J.A., Crippen, T.L., Knap, A.H., Sweet, S.T., Tomberlin, J.K., 2018. Larval digestion of different manure types by the black soldier fly (Diptera: Stratiomyidae) impacts associated volatile emissions. Waste Manag 74, 213-220.

Biancarosa, I., Liland, N.S., Biemans, D., Araujo, P., Bruckner, C.G., Waagbo, R., Torstensen, B.E., Lock, E.J., Amlund, H., 2018. Uptake of heavy metals and arsenic in black soldier fly (Hermetia illucens) larvae grown on seaweed-enriched media. J Sci Food Agric 98, 2176-2183.

Biancarosa, I., Sele, V., Belghit, I., Ornsrud, R., Lock, E.J., Amlund, H., 2019. Replacing fish meal with insect meal in the diet of Atlantic salmon (Salmo salar) does not impact the amount of contaminants in the feed and it lowers accumulation of arsenic in the fillet. Food Addit. Contam. Part A Chem. Anal. Control Expo. Risk Assess. 36, 1191-1205.

Biasato, I., Renna, M., Gai, F., Dabbou, S., Meneguz, M., Perona, G., Martinez, S., Lajusticia, A.C.B., Bergagna, S., Sardi, L., Capucchio, M.T., Bressan, E., Dama, A. Schiavone, A., Gasco, L., 2019. Partially defatted black soldier fly larva meal inclusion in piglet diets: effects on the growth performance, nutrient digestibility, blood profile, gut morphology and histological features. J Anim Sci Biotechnol 10.

Bloukounon-Goubalan, A.Y., Saïdou, A., Chrysostome, C.A.A.M., Kenis, M., Amadji, G. L., Igué, A.M., Mensah, G.A., 2019. Physical and Chemical Properties of the Agroprocessing By-products Decomposed by Larvae of Musca domestica and Hermetia illucens. Waste Biomass Valor 11, 2735-2743.

Bogner, J., Pipatti, R., Hashimoto, S., Diaz, C., Mareckova, K., Diaz, L., Kjeldsen, P., Monni, S., Faaij, A., Gao, Q., Zhang, T., Ahmed, M.A., Sutamihardja, R.T., Gregory, R., 2008. Mitigation of global greenhouse gas emissions from waste: conclusions and strategies from the Intergovernmental Panel on Climate Change (IPCC) Fourth Assessment Report. Working Group III (Mitigation) Waste Manag. Res. 26, 11-32.

Bondari, K., Sheppard, D.C., 1987. Soldier fly, Hermetia illucens L., larvae as feed for channel catfish, Ictalurus punctatus (Rafinesque), and blue tilapia, Oreochromis aureus (Steindachner). Aquacult Fish Manage 18, 209-220.

Bonelli, M., Bruno, D., Caccia, S., Sgambetterra, G., Cappellozza, S., Jucker, C. Tettamanti, G., Casartelli, M., 2019. Structural and Functional Characterization of Hermetia illucens Larval Midgut. Front. Physiol. 10, 204.

Borgogno, M., Dinnella, C., Iaconisi, V., Fusi, R., Scarpaleggia, C., Schiavone, A., Monteleone, E., Gasco, L., Parisi, G., 2017. Inclusion of Hermetia illucens larvae meal on rainbow trout (Oncorhynchus mykiss) feed: effect on sensory profile according to static and dynamic evaluations. J Sci Food Agric 97, 3402-3411.

Borrelli, L., Coretti, L., Dipineto, L., Bovera, F., Menna, F., Chiariotti, L., Nizza, A. Lembo, F., Fioretti, A., 2017. Insect-based diet, a promising nutritional source, modulates gut microbiota composition and SCFAs production in laying hens Sci. Rep. 7, 16269.

Bosch, G., Fels-Klerx, H.J.V., Rijk, T.C., Oonincx, D., 2017. Aflatoxin B1 Tolerance and Accumulation in Black Soldier Fly Larvae (Hermetia illucens) and Yellow Mealworms (Tenebrio molitor). Toxins (Basel) 9, 185.

Bosch, G., Oonincx, D.G.A.B., Jordan, H.R., Zhang, J., van Loon, J.J.A., van Huis, A. Tomberlin, J.K., 2020. Standardisation of quantitative resource conversion studies with black soldier fly larvae. J. Insects as Food Feed 6, 95-109.

Bosch, G., van Zanten, H.H.E., Zamprogna, A., Veenenbos, M., Meijer, N.P., van der Fels-Klerx, H.J., van Loon, J.J.A., 2019. Conversion of organic resources by black soldier fly larvae: Legislation, efficiency and environmental impact. J Clean Prod 222, 355-363.

Brede, A., Wecke, C., Liebert, F., 2018. Does the Optimal Dietary Methionine to Cysteine Ratio in Diets for Growing Chickens Respond to High Inclusion Rates of Insect Meal from Hermetia illucens? Animals (Basel) 8.

Brits, D., 2017. Improving feeding efficiencies of black soldier fly larvae, Hermetia illucens (L., 1758) (Diptera: Stratiomyidae: Hermetiinae) through manipulation of feeding conditions for industrial mass rearing, Faculty. In: of Conservation Ecology and Entomology. Stellenbosch University.

Bruni, L., Belghit, I., Lock, E.J., Secci, G., Taiti, C., Parisi, G., 2020. Total replacement of dietary fish meal with black soldier fly (Hermetia illucens) larvae does not impair physical, chemical or volatile composition of farmed Atlantic salmon (Salmo salar (L.)). J Sci Food Agric 100, 1038-1047.

Bruni, L., Pastorelli, R., Viti, C., Gasco, L., Parisi, G., 2018. Characterisation of the intestinal microbial communities of rainbow trout (Oncorhynchus mykiss) fed with Hermetia illucens (black soldier fly) partially defatted larva meal as partial dietary protein source. Aquaculture 487, 56-63.

Bruno, D., Bonelli, M., De Filippis, F., Di Lelio, I., Tettamanti, G., Casartelli, M., Ercolini, D., Caccia, S., 2019. The intestinal microbiota of Hermetia illucens larvae is affected by diet and shows a diverse composition in the different midgut regions. J Appl. Environ. Microbiol. 85, e01864-01818.

Bulak, P., Polakowski, C., Nowak, K., Wasko, A., Wiacek, D., Bieganowski, A., 2018. Hermetia illucens as a new and promising species for use in entomoremediation. Sci Total Environ 633, 912-919.

Bulak, P., Proc, K., Pawłowska, M., Kasprzycka, A., Berus, W., Bieganowski, A., 2020 Biogas generation from insects breeding post production wastes. J Clean Prod 244.

Cai, M., Hu, R., Zhang, K., Ma, S., Zheng, L., Yu, Z., Zhang, J., 2018. Resistance of black soldier fly (Diptera: Stratiomyidae) larvae to combined heavy metals and potential application in municipal sewage sludge treatment. Environ. Sci. Pollut. Res. Int. 25, 1559-1567.

Cai, M., Zhang, K., Zhong, W., Liu, N., Wu, X., Li, W., Zheng, L., Yu, Z., Zhang, J., 2017 Bioconversion-Composting of Golden Needle Mushroom (Flammulina velutipes) Root Waste by Black Soldier Fly (Hermetia illucens, Diptera: Stratiomyidae) Larvae, to Obtain Added-Value Biomass and Fertilizer. Waste Biomass Valor 10, 265-273.

Caimi, C., Renna, M., Lussiana, C., Bonaldo, A., Gariglio, M., Meneguz, M., Dabbou, S. Schiavone, A., Gai, F., Elia, A.C., Prearo, M., Gasco, L., 2020. First insights on Black Soldier Fly (Hermetia illucens (L.)) larvae meal dietary administration in Siberian sturgeon (Acipenser baerii Brandt) juveniles. Aquaculture 515.

Camenzuli, L., Van Dam, R., de Rijk, T., Andriessen, R., Van Schelt, J., Van der FelsKlerx, H.J.I., 2018. Tolerance and Excretion of the Mycotoxins Aflatoxin B(1) Zearalenone, Deoxynivalenol, and Ochratoxin A by Alphitobius diaperinus and Hermetia illucens from Contaminated Substrates. Toxins (Basel) 10, 91. 
Canakci, M., Sanli, H., 2008. Biodiesel production from various feedstocks and their effects on the fuel properties. J. Ind. Microbiol. Biotechnol. 35, 431-441.

Cardinaletti, G., Randazzo, B., Messina, M., Zarantoniello, M., Giorgini, E., Zimbelli, A., Bruni, L., Parisi, G., Olivotto, I., Tulli, F., 2019. Effects of Graded Dietary Inclusion Level of Full-Fat Hermetia illucens Prepupae Meal in Practical Diets for Rainbow Trout (Oncorhynchus mykiss). Animals (Basel) 9.

Caruso, D., Devic, E., Subamia, I.W., Talamond, P., Baras, E., 2013. Technica handbook of domestication and production of Diptera Black Soldier Fly (BSF), Hermetia illucens. PT Penerbit IPB Press, Stratiomyidae.

Caruso, D., Sholischah, L., Devic, E., 2013b. Economic Aspects of Production. In: Caruso, D., Devic, E., Subamia, I.W., Talamond, P., Baras, E. (Eds.), Technical handbook of domestication and production of diptera Black Soldier Fly (BSF) Hermetia illucens, Stratiomyidae. PT Penerbit IPB Press, Kampus IPB Taman Kencana Bogor, pp. 101-116.

Chen, J., Hou, D., Pang, W., Nowar, E.E., Tomberlin, J.K., Hu, R., Chen, H., Xie, J., Zhang J. Yu, Z., Li, Q., 2019. Effect of moisture content on greenhouse gas and $\mathrm{NH}_{3}$ emissions from pig manure converted by black soldier fly. Sci Total Environ 697, 133840.

Chernysh, S., Gordya, N., Suborova, T., 2015. Insect Antimicrobial Peptide Complexes Prevent Resistance Development in Bacteria. PLoS One 10, e0130788.

Chia, S.Y., Tanga, C.M., Osuga, I.M., Alaru, A.O., Mwangi, D.M., Githinji, M. Subramanian, S., Fiaboe, K.K.M., Ekesi, S., van Loon, J.J.A., Dicke, M., 2019. Effect of Dietary Replacement of Fishmeal by Insect Meal on Growth Performance, Blood Profiles and Economics of Growing Pigs in Kenya. Animals (Basel) 9, 705.

Choi, W.H., Choi, H.-J., Goo, T.W., Quan, F.-S., 2018. Novel antibacterial peptides induced by probiotics in Hermetia illucens (Diptera: Stratiomyidae) larvae. Entomol Res 48, 237-247.

Choi, Y.-C., Choi, J.-Y., Kim, J.-G., Kim, M.-S., Kim, W.-T., Park, K.-H., Bae, S.-W., Jeong G-S., 2009. Potential usage of food waste as a natural fertilizer after digestion by Hermetia illucens (Diptera: Stratiomyidae). Int J Indust Entomol 19, 171-174.

Chun, C.Y., Yoong, L.S., Kim, L.P., Hock, T.L., Ling, L.J., 2019. Comparison of Hermetia illucens larvae and pre-pupae as potential aqua feed derived from the biotransformation of organic waste. International Symposium on Green and Sustainable Technology (Isgst2019).

Churchward, C.P., Alany, R.G., Snyder, L.A.S., 2018. Alternative antimicrobials: the properties of fatty acids and monoglycerides. Crit Rev Microbiol 44, 561-570.

Cortes Ortiz, J.A., Ruiz, A.T., Morales-Ramos, J.A., Thomas, M., Rojas, M.G., Tomberlin, J.K., Yi, L., Han, R., Giroud, L., Jullien, R.L., 2016. Insect Mass Production Technologies. In: Dossey, A.T., Morales-Ramos, J.A., Rojas, M.G. (Eds.), Insects as Sustainable Food Ingredients. Academic Press, pp. 153-201.

Cullere, M., Schiavone, A., Dabbou, S., Gasco, L., Dalle, Z.A., 2019. Meat Quality and Sensory Traits of Finisher Broiler Chickens Fed with Black Soldier Fly (Hermetia Illucens (L.)) Larvae Fat as Alternative Fat Source. Animals (Basel) 9, 140.

Cullere, M., Tasoniero, G., Giaccone, V., Miotti-Scapin, R., Claeys, E., De Smet, S. Dalle, Z.A., 2016. Black soldier fly as dietary protein source for broiler quails: apparent digestibility, excreta microbial load, feed choice, performance, carcass and meat traits. Animal 10, 1923-1930.

Cutrignelli, M.I., Messina, M., Tulli, F., Randazzo, B., Olivotto, I., Gasco, L., Loponte, R. Bovera, F., 2018. Evaluation of an insect meal of the Black Soldier Fly (Hermetia illucens) as soybean substitute: Intestinal morphometry, enzymatic and microbial activity in laying hens. Res Vet Sci 117, 209-215.

da Silva, G.D.P., Hesselberg, T., 2020. A Review of the Use of Black Soldier Fly Larvae, Hermetia illucens (Diptera: Stratiomyidae), to Compost Organic Waste in Tropical Regions. Neotrop Entomol 49, 151-162.

Dabbou, S., Gai, F., Biasato, I., Capucchio, M.T., Biasibetti, E., Dezzutto, D., Meneguz, M., Placha, I., Gasco, L., Schiavone, A., 2018. Black soldier fly defatted meal as a dietary protein source for broiler chickens: Effects on growth performance, blood traits, gut morphology and histological features. J Anim Sci Biotechnol 9, 49.

Danieli, P.P. Lussiana, C. Gasco, L. Amici, A., Ronchi, B., 2019. The Effects of Diet Formulation on the Yield, Proximate Composition, and Fatty Acid Profile of the Black Soldier Fly (Hermetia illucens (L.)) Prepupae Intended for Animal Feed. Animals (Basel) 9, 178

De Smet, J., Wynants, E., Cos, P., Van Campenhout, L., 2018. Microbial community dynamics during rearing of black soldier fly larvae (Hermetia illucens) and impact on exploitation potential. J Appl. Environ. Microbiol. 84, e02722-02717.

Decuypere, J.A., Dierick, N.A., 2003. The combined use of triacylglycerols containing medium-chain fatty acids and exogenous lipolytic enzymes as an alternative to in-feed antibiotics in piglets: concept, possibilities and limitations. An overview. Nutr. Res. Rev. 16, 193-210.

Delicato, C., Schouteten, J.J., Dewettinck, K., Gellynck, X., Tzompa-Sosa, D.A., 2020. Consumers' perception of bakery products with insect fat as partial butter replacement. Food Qual. Prefer. 79, 103755.

Devic, E. Leschen, W. Murray, F., Little, D.C., 2018. Growth performance, feed utilization and body composition of advanced nursing Nile tilapia (Oreochromis niloticus) fed diets containing Black Soldier Fly (Hermetia illucens) larvae meal. Aquacult. Nutr. 24, 416-423.

Diener, S., 2010. Valorisation of organic solid waste using the black soldier fly, Hermetia illucens, in low and middle-income countries. ETH Zurich.

Diener, S., Studt Solano, N.M., Roa Gutiérrez, F., Zurbrügg, C., Tockner, K., 2011 Biological Treatment of Municipal Organic Waste using Black Soldier Fly Larvae. Waste Biomass Valor 2, 357-363.

Diener, S., Zurbrugg, C., Tockner, K., 2009. Conversion of organic material by black soldier fly larvae: establishing optimal feeding rates. Waste Manag. Res. 27, 603-610.
Diener, S., Zurbrügg, C., Tockner, K., 2015. Bioaccumulation of heavy metals in the black soldier fly, Hermetia illucens and effects on its life cycle. J Insects as Food Feed 1, 261-270.

Dortmans, B., 2015. Valorisation of organic waste-Effect of the feeding regime on process parameters in a continuous black soldier fly larvae composting system, Department of Energy and Technology. Swedish University of Agricultural Sciences.

Dortmans, B.M.A., Diener, S., Verstappen, B.M., Zurbrügg, C., 2017. Black Soldier Fly Biowaste Processing - A Step-by-Step Guide. Dübendorf, Switzerland.

Drew, D.J.W., Pieterse, E., 2015. Markets, money and maggots. J Insects as Food Feed 1, 227-231.

Dumas, A., Raggi, T., Barkhouse, J., Lewis, E., Weltzien, E., 2018. The oil fraction and partially defatted meal of black soldier fly larvae (Hermetia illucens) affect differently growth performance, feed efficiency, nutrient deposition, blood glucose and lipid digestibility of rainbow trout (Oncorhynchus mykiss). Aquaculture 492, 24-34.

Eilenberg, J., Vlak, J.M., Nielsen-LeRoux, C., Cappellozza, S., Jensen, A.B., 2015. Diseases in insects produced for food and feed. J Insects as Food Feed 1, 87-102.

Elhag, O., Zhou, D., Song, O., Soomro, A.A., Cai, M., Zheng, L., Yu, Z., Zhang, J., 2017. Screening, Expression, Purification and Functional Characterization of Novel Antimicrobial Peptide Genes from Hermetia illucens (L.). PLoS One 12, e0169582.

Erickson, M.C., Islam, M., Sheppard, C., Liao, J., Doyle, M.P., 2004. Reduction of Escherichia coli 0157: H7 and Salmonella enterica serovar Enteritidis in chicken manure by larvae of the black soldier fly. J. Food Prot. 67, 685-690.

Commission, European, 2001. Regulation (EC) No 999/2001 of the European Parliament and of the Council of 22 May 2001 laying down rules for the prevention, control and eradication of certain transmissible spongiform encephalopathies. Official Journal of the European Union 147, 1-40.

Commission, European, 2017. Commission regulation (EU) 2017/893 of 24 May 2017 amending annexes I and IV to Regulation (EC) No 999/2001 of the European parliament and of the council and annexes $\mathrm{X}, \mathrm{XIV}$ and $\mathrm{XV}$ to commission regulation (EU) No $142 / 2011$ as regards the provisions on processed animal protein. Official Journal of the European Union, 92-116.

Ewald, N., Vidakovic, A., Langeland, M., Kiessling, A., Sampels, S., Lalander, C., 2020. Fatty acid composition of black soldier fly larvae (Hermetia illucens)Possibilities and limitations for modification through diet. Waste Manage. $102,40-47$.

FAO, 2014. Mitigation of food wastage. Societal costs and benefits, Food and Agriculture Organization of the United Nations (FAO), Rome.

FAO, 2017. The future of food and agriculture - Trends and challenges, Rome.

FAO, 2018. The State of World Fisheries and Aquaculture 2018 - Meeting the sustainable development goals. Rome. Licence: CC BY-NC-SA 3.0 IGO.

Fawole, F.J., Adeoye, A.A., Tiamiyu, L.O., Ajala, K.I., Obadara, S.O., Ganiyu, I.O., 2020 Substituting fishmeal with Hermetia illucens in the diets of African catfish (Clarias gariepinus): Effects on growth, nutrient utilization, haemato-physiological response, and oxidative stress biomarker. Aquaculture 518, 734849.

Feng, W., Qian, L., Wang, W., Wang, T., Deng, Z., Yang, F., Xiong, J., Wang, C., 2018. Exploring the potential of lipids from black soldier fly: New paradigm for biodiesel production (II)-Extraction kinetics and thermodynamic. Renew Energ 119, 12-18.

Feng, W., Xiong, H., Wang, W., Duan, X., Yang, T., Wu, C., Yang, F., Wang, T., Wang, C., 2020. A facile and mild one-pot process for direct extraction of lipids from wet energy insects of black soldier fly larvae. Renew Energ 147, 584-593.

Feng, W., Xiong, H., Wang, W., Duan, X., Yang, T., Wu, C., Yang, F., Xiong, J., Wang, T., Wang, C., 2019. Energy consumption analysis of lipid extraction from black soldier fly biomass. Energy 185, 1076-1085.

Finke, M.D., 2007. Estimate of chitin in raw whole insects. Zoo Biol 26, 105-115.

Finke, M.D., 2013. Complete nutrient content of four species of feeder insects. Zoo Biol 32, 27-36.

Firmansyah, M., Abduh, M.Y., 2019. Production of protein hydrolysate containing antioxidant activity from Hermetia illucens. Heliyon 5, e02005.

Furman, D.P., Young, R.D., Catts, P.E., 1959. Hermetia illucens (Linnaeus) as a factor in the natural control of Musca domestica (Linnaeus). J Econ Entomol 52, 917-921.

Gao, Z., Wang, W., Lu, X., Zhu, F., Liu, W., Wang, X., Lei, C., 2019. Bioconversion performance and life table of black soldier fly (Hermetia illucens) on fermented maize straw. J Clean Prod 230, 974-980.

Gasco, L., Finke, M., van Huis, A., 2018. Can diets containing insects promote animal health? J Insects as Food Feed 4, 1-4.

Giannetto, A., Oliva, S., Ceccon Lanes, C.F., de Araujo Pedron, F., Savastano, D. Baviera, C., Parrino, V., Lo Paro, G., Spano, N.C., Cappello, T., Maisano, M., Mauceri, A., Fasulo, S., 2019. Hermetia illucens (Diptera: Stratiomydae) larvae and prepupae: Biomass production, fatty acid profile and expression of key genes involved in lipid metabolism. J Biotechnol 307, 44-54.

GlobeNewswire, 2018. Enterra receives new approvals to sell sustainable insect ingredients for animal feed in USA, Canada and EU, GlobeNewswire. GlobeNewswire, Inc

Gold, M., Cassar, C.M., Zurbrugg, C., Kreuzer, M., Boulos, S., Diener, S., Mathys, A., 2020. Biowaste treatment with black soldier fly larvae: Increasing performance through the formulation of biowastes based on protein and carbohydrates. Waste Manag 102, 319-329.

Gold, M., Tomberlin, J.K., Diener, S., Zurbrugg, C., Mathys, A., 2018. Decomposition of biowaste macronutrients, microbes, and chemicals in black soldier fly larval treatment: A review. Waste Manag 82, 302-318.

Green, T.R., Popa, R., 2012. Enhanced ammonia content in compost leachate processed by black soldier fly larvae. Appl. Biochem. Biotechnol. 166, 13811387. 
Grossule, V., Lavagnolo, M.C., 2019. The treatment of leachate using Black Soldier Fly (BSF) larvae: Adaptability and resource recovery testing. J Environ Manage 253, 109707.

Guerreiro, I., Castro, C., Antunes, B., Coutinho, F., Rangel, F., Couto, A., Serra, C.R., Peres, H., Pousão-Ferreira, P., Matos, E., Gasco, L., Gai, F., Corraze, G., Oliva-Teles, A., Enes, P., 2020. Catching black soldier fly for meagre: Growth, whole-body fatty acid profile and metabolic responses. Aquaculture 516, 734613.

Gulsunoglu, Z., Aravind, S., Bai, Y., Wang, L., Kutcher, H.R., Tanaka, T., 2019. Deoxynivalenol (DON) Accumulation and Nutrient Recovery in Black Soldier Fly Larvae (Hermetia illucens) Fed Wheat Infected with Fusarium spp. Fermentation 5,83 .

Hale, O.M., 1973. Dried Hermetia illucens larvae (Diptera: Stratiomyidae) as a feed additive for poultry. Ga Entomol Soc J.

Hem, S., Toure, S., Sagbla, C., Legendre, M., 2008. Bioconversion of palm kernel meal for aquaculture: Experiences from the forest region (Republic of Guinea). Afr J Biotechnol 7, 1192-1198.

Henry, M., Gasco, L., Piccolo, G., Fountoulaki, E., 2015. Review on the use of insects in the diet of farmed fish: Past and future. Anim. Feed Sci. Technol. 203, 1-22.

Hoc, B., Noel, G., Carpentier, J., Francis, F., Caparros Megido, R., 2019. Optimization of black soldier fly (Hermetia illucens) artificial reproduction. PLoS One 14, e0216160

Hu, J., Wang, G., Huang, Y., Sun, Y., He, F., Zhao, H., Li, N., 2017. Effects of substitution of fish meal with black soldier fly (Hermetia illucens) larvae meal, in yellow catfish (Pelteobagrus fulvidraco) diets. Israeli Journal of Aquaculture - Bamidgeh 69.

Huang, C., Feng, W., Xiong, J., Wang, T., Wang, W., Wang, C., Yang, F., 2018. Impact of drying method on the nutritional value of the edible insect protein from black soldier fly (Hermetia illucens (L.)) larvae: amino acid composition, nutritional value evaluation, in vitro digestibility, and thermal properties. Eur. Food Res. Technol. 245, 11-21.

Huyben, D., Vidaković, A., Werner Hallgren, S., Langeland, M., 2019. Highthroughput sequencing of gut microbiota in rainbow trout (Oncorhynchus mykiss) fed larval and pre-pupae stages of black soldier fly (Hermetia illucens). Aquaculture 500, 485-491.

IPIFF, 2019. EU Legislation. Brussels: International Platform of Insects for Food and Feed (IPIFF), Brussels, Belgium.

IPIFF, 2020. The European insect sector today: Challenges, opportunities and regulatory landscape. IPIFF vision paper on the future of the insect sector towards 2030. The International Platform of Insects for Food and Feed, Brussels, Belgium.

Ishak, S., Kamari, A., 2019. Biodiesel from black soldier fly larvae grown on restaurant kitchen waste. Environ. Chem. Lett. 17, 1143-1150.

Isibika, A., Vinneras, B., Kibazohi, O., Zurbrugg, C., Lalander, C., 2019. Pre-treatment of banana peel to improve composting by black soldier fly (Hermetia illucens (L.), Diptera: Stratiomyidae) larvae. Waste Manag 100, 151-160.

Janssen, R.H., Canelli, G., Sanders, M.G., Bakx, E.J., Lakemond, C.M.M., Fogliano, V., Vincken, J.P., 2019. Iron-polyphenol complexes cause blackening upon grinding Hermetia illucens (black soldier fly) larvae. Sci. Rep. 9, 2967.

Janssen, R.H., Vincken, J.P., van den Broek, L.A., Fogliano, V., Lakemond, C.M., 2017. Nitrogen-to-Protein Conversion Factors for Three Edible Insects: Tenebrio molitor, Alphitobius diaperinus, and Hermetia illucens. J. Agric. Food Chem. 65, 2275-2278.

Jeon, H., Park, S., Choi, J., Jeong, G., Lee, S.B., Choi, Y., Lee, S.J., 2011. The intestinal bacterial community in the food waste-reducing larvae of Hermetia illucens. Curr. Microbiol. 62, 1390-1399.

Ji, Y.J., Liu, H.N., Kong, X.F., Blachier, F., Geng, M.M., Liu, Y.Y., Yin, Y.L., 2016. Use of insect powder as a source of dietary protein in early-weaned piglets. J. Anim. Sci. 94, 111-116.

Joly, G., Nikiema, J., 2019. Global experiences on waste processing with black soldier fly (Hermetia illucens): from technology to business. Colombo, Sri Lanka: International Water Management Institute (IWMI). CGIAR Research Program on Water, Land and Ecosystems (WLE) 62p (Resource Recovery and Reuse Series 16).

Jucker, C., Erba, D., Leonardi, M.G., Lupi, D., Savoldelli, S., 2017. Assessment of Vegetable and Fruit Substrates as Potential Rearing Media for Hermetia illucens (Diptera: Stratiomyidae) Larvae. Environ Entomol 46, 1415-1423.

Jucker, C., Lupi, D., Moore, C.D., Leonardi, M.G., Savoldelli, S., 2020. Nutrient Recapture from Insect Farm Waste: Bioconversion with Hermetia illucens (L.). (Diptera: Stratiomyidae). Sustainability 12, 362.

Julita, U., Suryani, Y., Kinasih, I., Yuliawati, A., Cahyanto, T., Maryeti, Y., Permana, A. D., Fitri, L.L., 2018. Growth performance and nutritional composition of black soldier fly, Hermetia illucens (L), (Diptera: Stratiomyidae) reared on horse and sheep manure. IOP Conference Series: Earth and Environmental Science 187, 012071.

Kamarulzaman, M.K. Hafiz, M., Abdullah, A. Chen, A.F., Awad, O.I. 2019. Combustion, performances and emissions characteristics of black soldier fly larvae oil and diesel blends in compression ignition engine. Renew Energ 142, 569-580.

Karlsen, Ø., Amlund, H., Berg, A., Olsen, R.E., 2017. The effect of dietary chitin on growth and nutrient digestibility in farmed Atlantic cod, Atlantic salmon and Atlantic halibut. Aquacult. Res. 48, 123-133.

Kawasaki, K., Hashimoto, Y., Hori, A., Kawasaki, T., Hirayasu, H., Iwase, S.I., Hashizume, A., Ido, A., Miura, C., Miura, T., Nakamura, S., Seyama, T., Matsumoto, Y., Kasai, K., Fujitani, Y., 2019. Evaluation of Black Soldier Fly (Hermetia illucens) Larvae and Pre-Pupae Raised on Household Organic Waste, as Potential Ingredients for Poultry Feed. Animals (Basel) 9, 98.
Kaza, S., Yao, L., Perinaz, B.-T., Van Woerden, F., 2018. What a Waste 2.0: A Global Snapshot of Solid Waste Management to 2050. The World Bank.

Kenis, M., Bouwassi, B., Boafo, H., Devic, E., Han, R., Koko, G., Koné, N.G., MacielVergara, G., Nacambo, S., Pomalegni, S.C.B., Roffeis, M., Wakefield, M., Zhu, F. Fitches, E., 2018. Small-Scale Fly Larvae Production for Animal Feed. In: Halloran, A., Flore, R., Vantomme, P., Roos, N. (Eds.), Edible Insects in Sustainable Food Systems. Springer, Cham, pp. 239-261.

Khempaka, S., Chitsatchapong, C., Molee, W., 2011. Effect of chitin and protein constituents in shrimp head meal on growth performance, nutrient digestibility, intestinal microbial populations, volatile fatty acids, and ammonia production in broilers. J Appl Poultry Res 20, 1-11.

Kierończyk, B., Sypniewski, J., Rawski, M., Czekała, W., Świątkiewicz, S., Józefiak, D. 2019. From waste to sustainable feed material: the effect of Hermetia illucens oil on the growth performance, nutrient digestibility, and gastrointestinal tract morphometry of broiler chickens. Ann Anim Sci 20, 157-177.

Kim, W., Bae, S., Park, K., Lee, S., Choi, Y., Han, S., Koh, Y., 2011. Biochemical characterization of digestive enzymes in the black soldier fly, Hermetia illucens (Diptera: Stratiomyidae). J. Asia-Pacif. Entomol. 14, 11-14.

Komi, D.E.A., Sharma, L., Dela Cruz, C.S., 2018. Chitin and Its Effects on Inflammatory and Immune Responses. Clin. Rev. Allergy Immunol. 54, 213-223.

Kroeckel, S., Harjes, A.G.E., Roth, I., Katz, H., Wuertz, S., Susenbeth, A., Schulz, C. 2012. When a turbot catches a fly: Evaluation of a pre-pupae meal of the Black Soldier Fly (Hermetia illucens) as fish meal substitute - Growth performance and chitin degradation in juvenile turbot (Psetta maxima). Aquaculture 364365, 345-352.

Lalander, C., Diener, S., Magri, M.E., Zurbrugg, C., Lindstrom, A., Vinneras, B., 2013. Faecal sludge management with the larvae of the black soldier fly (Hermetia illucens) - from a hygiene aspect. Sci Total Environ 458-460, 312-318.

Lalander, C., Diener, S., Zurbrügg, C., Vinnerås, B., 2019. Effects of feedstock on larval development and process efficiency in waste treatment with black soldier fly (Hermetia illucens). J Clean Prod 208, 211-219.

Lalander, C., Senecal, J., Gros Calvo, M., Ahrens, L., Josefsson, S., Wiberg, K., Vinneras, B., 2016. Fate of pharmaceuticals and pesticides in fly larvae composting. Sci Total Environ 565, 279-286.

Lalander, C.H., Fidjeland, J., Diener, S., Eriksson, S., Vinnerås, B., 2015. High waste-tobiomass conversion and efficient Salmonella spp. reduction using black soldier fly for waste recycling. Agron Sustain Dev 35, 261-271.

Larouche, J., Deschamps, M.H., Saucier, L., Lebeuf, Y., Doyen, A., Vandenberg, G.W. 2019. Effects of Killing Methods on Lipid Oxidation, Colour and Microbial Load of Black Soldier Fly (Hermetia illucens) Larvae. Animals (Basel) 9, 182.

Lee, C.G., Da Silva, C.A., Lee, J.Y., Hartl, D., Elias, J.A., 2008. Chitin regulation of immune responses: an old molecule with new roles. Curr. Opin. Immunol. 20 684-689.

Lee, C.M., Lee, Y.S., Seo, S.H., Yoon, S.H., Kim, S.J., Hahn, B.S., Sim, J.S., Koo, B.S., 2014 Screening and characterization of a novel cellulase gene from the gut microflora of Hermetia illucens using metagenomic library. J. Microbiol. Biotechnol. 24 $1196-1206$.

Lee, J., Kim, Y.M., Park, Y.K., Yang, Y.C., Jung, B.G., Lee, B.J., 2018. Black soldier fly (Hermetia illucens) larvae enhances immune activities and increases survivability of broiler chicks against experimental infection of Salmonella Gallinarum. J Vet Med Sci 80, 736-740.

Leni, G., Caligiani, A., Sforza, S., 2019. Killing method affects the browning and the quality of the protein fraction of Black Soldier Fly (Hermetia illucens) prepupae: a metabolomics and proteomic insight. Food Res. Int. 115, 116-125.

Leong, S.Y., Kutty, S.R., Malakahmad, A., Tan, C.K., 2016. Feasibility study of biodiesel production using lipids of Hermetia illucens larva fed with organic waste. Waste Manage. 47, 84-90.

Li, Q., Zheng, L., Cai, H., Garza, E., Yu, Z., Zhou, S., 2011. From organic waste to biodiesel: Black soldier fly, Hermetia illucens, makes it feasible. Fuel 90, 15451548.

Li, Q., Zheng, L., Oiu, N., Cai, H., Tomberlin, J.K., Yu, Z., 2011b. Bioconversion of dairy manure by black soldier fly (Diptera: Stratiomyidae) for biodiesel and sugar production. Waste Manag 31, 1316-1320.

Li, S., Ji, H., Zhang, B., Tian, J., Zhou, J., Yu, H., 2016. Influence of black soldier fly (Hermetia illucens) larvae oil on growth performance, body composition, tissue fatty acid composition and lipid deposition in juvenile Jian carp (Cyprinus carpio var. Jian). Aquaculture 465, 43-52.

Li, S., Ji, H., Zhang, B., Zhou, J., Yu, H., 2017. Defatted black soldier fly (Hermetic illucens) larvae meal in diets for juvenile Jian carp (Cyprinus carpio var. Jian): Growth performance, antioxidant enzyme activities, digestive enzyme activities intestine and hepatopancreas histological structure. Aquaculture 477, 62-70.

Li, W., Li, M., Zheng, L., Liu, Y., Zhang, Y., Yu, Z., Ma, Z., Li, O., 2015. Simultaneous utilization of glucose and xylose for lipid accumulation in black soldier fly. Biotechnol Biofuels 8, 117.

Liland, N.S., Biancarosa, I., Araujo, P., Biemans, D., Bruckner, C.G., Waagbo, R. Torstensen, B.E., Lock, E.J., 2017. Modulation of nutrient composition of black soldier fly (Hermetia illucens) larvae by feeding seaweed-enriched media. PLoS One 12, e0183188.

Lim, J.W., Mohd-Noor, S.N., Wong, C.Y., Lam, M.K., Goh, P.S., Beniers, J.J.A., Oh, W.D. Jumbri, K., Ghani, N.A., 2019. Palatability of black soldier fly larvae in valorizing mixed waste coconut endosperm and soybean curd residue into larval lipid and protein sources. J Environ Manage 231, 129-136.

Liu, Q., Tomberlin, J.K., Brady, J.A., Sanford, M.R., Yu, Z., 2008. Black soldier fly (Diptera: Stratiomyidae) larvae reduce Escherichia coli in dairy manure. Environ. Entomol. 37, 1525-1530. 
Liu, T., Awasthi, M.K., Chen, H., Duan, Y., Awasthi, S.K., Zhang, Z., 2019. Performance of black soldier fly larvae (Diptera: Stratiomyidae) for manure composting and production of cleaner compost. J Environ Manage 251, 109593.

Liu, X., Chen, X., Wang, H., Yang, Q., Ur Rehman, K., Li, W., Cai, M., Li, Q., Mazza, L., Zhang, J., Yu, Z., Zheng, L., 2017. Dynamic changes of nutrient composition throughout the entire life cycle of black soldier fly. PLoS One 12, e0182601.

Lock, E.R., Arsiwalla, T., Waagbø, R., 2016. Insect larvae meal as an alternative source of nutrients in the diet of Atlantic salmon (Salmo salar) postsmolt. Aquacult. Nutr. 22, 1202-1213.

Lohri, C.R., Diener, S., Zabaleta, I., Mertenat, A., Zurbrügg, C., 2017. Treatment technologies for urban solid biowaste to create value products: a review with focus on low- and middle-income settings. Reviews in Environmental Science and Bio/Technology 16, 81-130.

Magalhães, R., Sánchez-López, A., Leal, R.S., Martínez-Llorens, S., Oliva-Teles, A. Peres, H., 2017. Black soldier fly (Hermetia illucens) pre-pupae meal as a fish meal replacement in diets for European seabass (Dicentrarchus labrax). Aquaculture 476, 79-85.

Mai, H.C., Dao, N.D., Lam, T.D., Nguyen, B.V., Nguyen, D.C., Bach, L.G., 2019. Purification Process, Physicochemical Properties, and Fatty Acid Composition of Black Soldier Fly (Hermetia illucens (Linnaeus)) Larvae Oil. J. Am. Oil Chem. Soc. 96, 1303-1311.

Makkar, H.P.S., Tran, G., Heuzé, V., Ankers, P., 2014. State-of-the-art on use of insects as animal feed. Anim. Feed Sci. Technol. 197, 1-33.

Mamai, W., Bimbile Somda, N.S., Maiga, H., Konczal, A., Wallner, T., Bakhoum, M.T., Yamada, H., Bouyer, J., 2019. Black soldier fly (Hermetia illucens) larvae powde as a larval diet ingredient for mass-rearing Aedes mosquitoes. Parasite 26, 57.

Mancini, S., Medina, I., Iaconisi, V., Gai, F., Basto, A., Parisi, G., 2018. Impact of black soldier fly larvae meal on the chemical and nutritional characteristics of rainbow trout fillets. Animal 12, 1672-1681.

Marono, S., Loponte, R., Lombardi, P., Vassalotti, G., Pero, M.E., Russo, F., Gasco, L., Parisi, G., Piccolo, G., Nizza, S., Di Meo, C., Attia, Y.A., Bovera, F., 2017. Productive performance and blood profiles of laying hens fed Hermetia illucens larvae meal as total replacement of soybean meal from 24 to 45 weeks of age. Poult Sci 96, $1783-1790$.

Marono, S., Piccolo, G., Loponte, R., Di Meo, C., Attia, Y.A., Nizza, A., Bovera, F., 2016 In Vitro Crude Protein Digestibility of Tenebrio molitor and Hermetia illucens Insect Meals and its Correlation with Chemical Composition Traits. Ital J Anim Sci 14,3889

Martín-Folgar, R., Martínez-Guitarte, J.-L., 2017. Cadmium alters the expression of small heat shock protein genes in the aquatic midge Chironomus riparius. Chemosphere 169, 485-492.

Matheson, T., 2019. Disposal is Not Free: Fiscal Instruments to Internalize the Environmental Costs of Solid Waste. International Monetary Fund.

Matthäus, B., Piofczyk, T., Katz, H., Pudel, F., 2019. Renewable Resources from Insects: Exploitation, Properties, and Refining of Fat Obtained by Cold-Pressing from Hermetia illucens (Black Soldier Fly). Larvae. European Journal of Lipid Science and Technology 121

Maurer, V., Holinger, M., Amsler, Z., Früh, B., Wohlfahrt, J., Stamer, A., Leiber, F., 2016. Replacement of soybean cake by Hermetia illucens meal in diets for layers. I Insects as Food Feed 2, 83-90.

May, B.M., 1961. The occurrence in New Zealand and the life-history of the soldier fly Hermetia illucens (L.) (Diptera: Stratiomyidae). NZ J Sci 4, 55-65.

Mazza, L., Xiao, X., ur Rehman, K., Cai, M., Zhang, D., Fasulo, S., Tomberlin, J.K., Zheng, L., Soomro, A.A., Yu, Z., Zhang, J., 2020. Management of chicken manure using black soldier fly (Diptera: Stratiomyidae) larvae assisted by companion bacteria. Waste Manage. 102, 312-318.

Mbhele, F.G.T., Mnisi, C.M., Mlambo, V., 2019. A Nutritional Evaluation of Insect Meal as a Sustainable Protein Source for Jumbo Quails: Physiological and Meat Quality Responses. Sustainability 11, 6592.

McDougal, T., 2018. US: Black soldier fly larvae for poultry gets green light. Poultry World.

Meijer, N., Stoopen, G., van der Fels-Klerx, H.J., van Loon, J.J.A., Carney, J., Bosch, G., 2019. Aflatoxin B1 Conversion by Black Soldier Fly (Hermetia illucens). Larval Enzyme Extracts. Toxins Basel, 11.

Meneguz, M., Schiavone, A., Gai, F., Dama, A., Lussiana, C., Renna, M., Gasco, L., 2018. Effect of rearing substrate on growth performance, waste reduction efficiency and chemical composition of black soldier fly (Hermetia illucens) larvae. J Sci Food Agric 98, 5776-5784.

Mertenat, A., Diener, S., Zurbrugg, C., 2019. Black Soldier Fly biowaste treatment Assessment of global warming potential. Waste Manag 84, 173-181.

Miranda, C.D., Cammack, J.A., Tomberlin, J.K., 2019. Life-History Traits of the Black Soldier Fly, Hermetia illucens (L.) (Diptera: Stratiomyidae), Reared on Three Manure Types. Animals 9.

Mohd-Noor, S.-N., Wong, C.-Y., Lim, J.-W., Mah-Hussin, M.-I.-A., Uemura, Y., Lam, M.-K., Ramli, A., Bashir, M.J.K., Tham, L., 2017. Optimization of self-fermented period of waste coconut endosperm destined to feed black soldier fly larvae in enhancing the lipid and protein yields. Renew Energ 111, 646-654.

Moula, N., Scippo, M.L., Douny, C., Degand, G., Dawans, E., Cabaraux, J.F., Hornick, J. L., Medigo, R.C., Leroy, P., Francis, F., Detilleux, J., 2018. Performances of loca poultry breed fed black soldier fly larvae reared on horse manure. Anim Nutr 4, 73-78.

Muin, H., Taufek, N.M., Kamarudin, M.S., Razak, S.A., 2017. Growth performance, feed utilization and body composition of Nile tilapia, Reochromis niloticus (Linnaeus, 1758) fed with different levels of black soldier fly, Hermetia illucens (Linnaeus, 1758) maggot meal diet. Iran. J. Fish. Sci. 16, 567-577.
Muller, A., Wiedmer, S., Kurth, M., 2019. Risk Evaluation of Passive Transmission of Animal Parasites by Feeding of Black Soldier Fly (Hermetia illucens) Larvae and Prepupae. J. Food Prot. 82, 948-954.

Muller, A., Wolf, D., Gutzeit, H.O., 2017. The black soldier fly, Hermetia illucens - a promising source for sustainable production of proteins, lipids and bioactive substances. Z. Naturforsch. C 72, 351-363.

Mwaniki, Z., Neijat, M., Kiarie, E., 2018. Egg production and quality responses of adding up to $7.5 \%$ defatted black soldier fly larvae meal in a corn-soybean meal diet fed to Shaver White Leghorns from wk 19 to 27 of age. Poult Sci 97, 28292835.

Mwaniki, Z.N., Kiarie, E., Plaizier, J., 2019. Standardized ileal digestible amino acids and apparent metabolizable energy content in defatted black soldier fly larvae meal fed to broiler chickens. Can J Anim Sci 99, 211-217.

Myers, H.M., Tomberlin, J.K., Lambert, B.D., Kattes, D., 2008. Development of Black Soldier Fly (Diptera: Stratiomyidae) Larvae Fed Dairy Manure. Environ. Entomol. 37, 11-15.

Nekrasov, R.V., Pravdin, I.V., Kravtsova, L.Z., Bastrakov, A.I., Pashkova, L.A., Ushakova, N.A., 2015. Biochemical characteristics of Hermetia illucens: A base for prospective use of larval biomass in young pig food. J Nat Sci Sustain Technol 9, 407-416.

Nekrasov, R.V., Zelenchenkova, A.A., Chabaev, M.G., Ivanov, G.A., Antonov, A.M., Pastukhova, N.O., 2018. Dried Black Soldier Fly larvae as a dietary supplement to the diet of growing pigs. J. Anim. Sci. 96, 314

Nery, J., Gasco, L., Dabbou, S., Schiavone, A., 2018. Protein composition and digestibility of black soldier fly larvae in broiler chickens revisited according to the recent nitrogen-protein conversion ratio. J Insects as Food Feed 4, 171-177.

Newton, G.L., Booram, C.V., Barker, R.W., Hale, O.M., 1977. Dried Hermetia illucens larvae meal as a supplement for swine. J. Anim. Sci. 44, 395-400.

Newton, L., Sheppard, C., Watson, W., Burtle, G., Robert, D., 2005. Using the black soldier fly, Hermetia illucens, as a value-added tool for the management of swine manure. Animal. In: and Poultry Waste Management. Center, North Carolina State University, Raleigh, NC, USA.

Nguyen, H.C., Liang, S.-H., Chen, S.-S., Su, C.-H., Lin, J.-H., Chien, C.-C., 2018 Enzymatic production of biodiesel from insect fat using methyl acetate as an acyl acceptor: Optimization by using response surface methodology. Energy Convers. Manage. 158, 168-175.

Nguyen, H.C., Nguyen, M.L., Liang, S.-H., Su, C.-H., Wang, F.-M., 2020. Switchable Solvent-Catalyzed Direct Transesterification of Insect Biomass for Biodiesel Production. Bioenerg. Res. 13, 563-570.

Nguyen, T.T., Tomberlin, J.K., Vanlaerhoven, S., 2015. Ability of Black Soldier Fly (Diptera: Stratiomyidae) Larvae to Recycle Food Waste. Environ Entomol 44, 406-410.

Nguyen, T.T.X., Tomberlin, J.K., Vanlaerhoven, S., 2013. Influence of Resources on Hermetia illucens (Diptera: Stratiomyidae) Larval Development. J. Med. Entomol. 50, 898-906.

Nyakeri, E.M., Ayieko, M.A., Amimo, F.A., Salum, H., Ogola, H.J.O., 2019. An optimal feeding strategy for black soldier fly larvae biomass production and faecal sludge reduction. J Insects Food Feed 5, 201-213.

Nyakeri, E.M., Ogola, H.J., Ayieko, M.A., Amimo, F.A., 2017a. An open system for farming black soldier fly larvae as a source of proteins for small scale poultry and fish production. J Insects as Food Feed 3, 51-56.

Nyakeri, E.M., Ogola, H.J.O., Ayieko, M.A., Amimo, F.A., 2017b. Valorisation of organic waste material: growth performance of wild black soldier fly larvae (Hermetia illucens) reared on different organic wastes. J Insects as Food Feed 3, 193-202.

Ochoa-Sanabria, C., 2019. Feeding mycotoxin-contaminated wheat to insect larvae (Tenebrio molitor) as a strategy to use Fusarium damaged grain and generate a safe protein replacement for animal feed. Bulletin of the Entomological Society of Canada 50, 31-35.

Ogejo, J.A., 2018. Selecting a Treatment Technology for Manure Management. In: Extension, V.C. (Ed.), Virginia Tech. Virginia State University.

Oliveira, F., Doelle, K., List, R., O'Reilly, J.R., 2015. Assessment of Diptera: Stratiomyidae, genus Hermetia illucens (L., 1758) using electron microscopy. J. Entomol. Zool. Stud. 3, 147-152.

Onsongo, V.O., Osuga, I.M., Gachuiri, C.K., Wachira, A.M., Miano, D.M., Tanga, C.M., Ekesi, S., Nakimbugwe, D., Fiaboe, K.K.M., 2018. Insects for Income Generation Through Animal Feed: Effect of Dietary Replacement of Soybean and Fish Meal With Black Soldier Fly Meal on Broiler Growth and Economic Performance. J Econ Entomol 111, 1966-1973.

Oonincx, D.G.A.B., van Huis, A., van Loon, J.J.A., 2015. Nutrient utilisation by black soldier flies fed with chicken, pig, or cow manure. J. Insects as Food Feed. 1, 131-139.

Palma, L., Ceballos, S.J., Johnson, P.C., Niemeier, D., Pitesky, M., VanderGheynst, J.S., 2018. Cultivation of black soldier fly larvae on almond byproducts: impacts of aeration and moisture on larvae growth and composition. J Sci Food Agric 98, $5893-5900$

Palma, L., Fernandez-Bayo, J., Niemeier, D., Pitesky, M., VanderGheynst, J.S., 2019. Managing high fiber food waste for the cultivation of black soldier fly larvae. NPJ Sci Food 3, 15

Pang, W., Hou, D., Ke, J., Chen, J., Holtzapple, M.T., Tomberlin, J.K., Chen, H., Zhang, J., $\mathrm{Li}, \quad$ Q. 2019. Production of biodiesel from $\mathrm{CO}_{2}$ and organic wastes by fermentation and black soldier fly. Renew. Energy 149, 1174-1181.

Park, H.H., 2016. Black soldier fly larvae manual. Student Showcase 14.

Park, K.H., Choi, Y.C., Nam, S.H., Kim, W.T., Kim, A.Y., Kim, S.Y., 2012. Recombinant Expression and Enzyme Activity of Chymotrypsin-like Protease from Black Soldier Fly, Hermetia illucens (Diptera: Stratiomyidae). Int J Ind Entomol 25, 181-185. 
Park, S.-I., Yoe, S.M., 2017. A novel cecropin-like peptide from black soldier fly, Hermetia illucens: Isolation, structural and functional characterization. Entomol Res 47, 115-124.

Park, S.I., Kim, J.W., Yoe, S.M., 2015. Purification and characterization of a novel antibacterial peptide from black soldier fly (Hermetia illucens) larvae. Dev Comp Immunol 52, 98-106.

Parra Paz, A.S., Carrejo, N.S., Gómez Rodríguez, C.H., 2015. Effects of Larval Density and Feeding Rates on the Bioconversion of Vegetable Waste Using Black Soldier Fly Larvae Hermetia illucens (L.), (Diptera: Stratiomyidae). Waste Biomass Valor 6, 1059-1065.

Pieterse, E., Erasmus, S.W., Uushona, T., Hoffman, L.C., 2019. Black soldier fly (Hermetia illucens) pre-pupae meal as a dietary protein source for broiler production ensures a tasty chicken with standard meat quality for every pot. J Sci Food Agric 99, 893-903.

Pimentel, A.C., Montali, A., Bruno, D., Tettamanti, G., 2017. Metabolic adjustment of the larval fat body in Hermetia illucens to dietary conditions. J. Asia-Pacif. Entomol. 20, 1307-1313.

Pimentel, D., Pimentel, M., 2003. Sustainability of meat-based and plant-based diets and the environment. Am. J. Clin. Nutr. 78, 660S-663S.

Pinotti, L., Ottoboni, M., Luciano, A., Savoini, G., Cattaneo, D., Tretola, M., 2019. Exfood in animal nutrition: potentials and challenges. Wageningen Academic Publishers, EAAP Scientific Series, p. 1064580.

Pleissner, D., Smetana, S., 2019. Estimation of the economy of heterotrophic microalgae- and insect-based food waste utilization processes. Waste Manag $102,198-203$.

Popa, R., Green, T.R., 2012. Using Black Soldier Fly Larvae for Processing Organic Leachates. J. Econ. Entomol. 105, 374-378.

Purkayastha, D., Sarkar, S., 2019. Physicochemical Structure Analysis of Chitin Extracted from Pupa Exuviae and Dead Imago of Wild Black Soldier Fly (Hermetia illucens). J. Polym. Environ. 28, 445-457.

Purschke, B., Scheibelberger, R., Axmann, S., Adler, A., Jager, H., 2017. Impact of substrate contamination with mycotoxins, heavy metals and pesticides on the growth performance and composition of black soldier fly larvae (Hermetia illucens) for use in the feed and food value chain. Food Addit. Contam. Part A Chem. Anal. Control Expo. Risk Assess. 34, 1410-1420.

Rabani, V., Cheatsazan, H., Davani, S., 2019. Proteomics and Lipidomics of Black Soldier Fly (Diptera: Stratiomyidae) and Blow Fly (Diptera: Calliphoridae) Larvae. J Insect Sci 19.

Rana, K.S., Salam, M.A., Hashem, S., Islam, M.A., 2015. Development of black soldier fly larvae production technique as an alternate fish feed. Int. J. Res. Fish. Aquacult 5, 41-47.

Ravi, H.K., Vian, M.A., Tao, Y., Degrou, A., Costil, J., Trespeuch, C., Chemat, F., 2019. Alternative solvents for lipid extraction and their effect on protein quality in black soldier fly (Hermetia illucens) larvae. J Clean Prod 238.

Razdan, A., Pettersson, D., 1994. Effect of chitin and chitosan on nutrient digestibility and plasma lipid concentrations in broiler chickens. Br. J. Nutr. 72, 277-288.

Reddy, P.S., Nandini, N., 2011. Leachate characterization and assessment of groundwater pollution near municipal solid waste landfill site. Nature, Environment Pollution Technology 10, 415-418.

Rehman, H., Vahjen, W., Kohl-Parisini, A., Ijaz, A., Zentek, J., 2009. Influence of fermentable carbohydrates on the intestinal bacteria and enteropathogens in broilers. Worlds Poult, Sci. J, p. 65.

Rehman, K.u., Liu, X., Wang, H., Zheng, L., Rehman, R.u., Cheng, X., Li, Q., Li, W., Cai, M., Zhang, J., Yu, Z., 2018. Effects of black soldier fly biodiesel blended with diesel fuel on combustion, performance and emission characteristics of diesel engine. Energy Convers. Manage. 173, 489-498.

Rehman, K.U., Cai, M., Xiao, X., Zheng, L., Wang, H., Soomro, A.A., Zhou, Y., Li, W., Yu, Z., Zhang, J., 2017. Cellulose decomposition and larval biomass production from the co-digestion of dairy manure and chicken manure by mini-livestock (Hermetia illucens (L.)). J Environ Manage 196, 458-465.

Rehman, K.U., Rehman, A., Cai, M., Zheng, L., Xiao, X., Somroo, A.A., Wang, H., Li, W., Yu, Z., Zhang, J., 2017. Conversion of mixtures of dairy manure and soybean curd residue by black soldier fly larvae (Hermetia illucens (L.)). J Clean Prod 154, 366373.

Rehman, K.U., Ur Rehman, R., Somroo, A.A., Cai, M., Zheng, L., Xiao, X., Ur Rehman, A., Rehman, A., Tomberlin, J.K., Yu, Z., Zhang, J., 2019. Enhanced bioconversion of dairy and chicken manure by the interaction of exogenous bacteria and black soldier fly larvae. J Environ Manage 237, 75-83.

Renna, M., Schiavone, A., Gai, F., Dabbou, S., Lussiana, C., Malfatto, V., Prearo, M., Capucchio, M.T., Biasato, I., Biasibetti, E., De Marco, M., Brugiapaglia, A., Zoccarato, I., Gasco, L., 2017. Evaluation of the suitability of a partially defatted black soldier fly (Hermetia illucens (L.)) larvae meal as ingredient for rainbow trout (Oncorhynchus mykiss Walbaum) diets. J Anim Sci Biotechnol 8, 57.

Rimoldi, S., Gini, E., Iannini, F., Gasco, L., Terova, G., 2019. The Effects of Dietary Insect Meal from Hermetia illucens Prepupae on Autochthonous Gut Microbiota of Rainbow Trout (Oncorhynchus mykiss). Animals (Basel) 9.

Roffeis, M., Fitches, E.C., Wakefield, M.E., Almeida, J., Alves Valada, T.R., Devic, E., Koné, N.G., Kenis, M., Nacambo, S., Koko, G.K.D., Mathijs, E., Achten, W.M.J., Muys, B., 2020. Ex-ante life cycle impact assessment of insect based feed production in West Africa. Agricultural Systems 178.

Roffeis, M., Wakefield, M.E., Almeida, J., Alves Valada, T.R., Devic, E., Koné, N.G., Kenis, M., Nacambo, S., Fitches, E.C., Koko, G.K.D., Mathijs, E., Achten, W.M.J., Muys, B., 2018. Life cycle cost assessment of insect based feed production in West Africa. J Clean Prod 199, 792-806.
Roháček, J., Hora, M., 2013. A northernmost European record of the alien black soldier fly Hermetia illucens (Linnaeus, 1758) (Diptera: Stratiomyidae). Casopis slezskeho zemskeho muzea 62, 101-106.

Rosmiati, M., Nurjanah, K.A., Suantika, G., Putra, R.E., 2017. Application of compost produced by bioconversion of coffee husk by black soldier fly larvae (Hermetia illucens) as solid fertilizer to lettuce (Lactuca sativa var. crispa): impact to growth. International Conference on Green Technology, 38-44.

Ruhnke, I., Normant, C., Campbell, D.L.M., Iqbal, Z., Lee, C., Hinch, G.N., Roberts, J., 2018. Impact of on-range choice feeding with black soldier fly larvae (Hermetia illucens) on flock performance, egg quality, and range use of free-range laying hens. Anim Nutr 4, 452-460.

Rumpold, B., Speckmann, H., Schlüter, O., Kloas, W., Prochnow, A. 2018. Potentials of a biogenic residue-based production of Hermetia illucens as fish mea replacement in aquafeed for Oncorhynchus mykiss in Germany. J. Insects as Food Feed 4, 5-18.

Rumpold, B.A., Schlüter, O.K., 2013. Potential and challenges of insects as an innovative source for food and feed production. Innov. Food Sci. Emerg. Technol. 17, 1-11.

Russ, W. Meyer-Pittroff, R. 2004. Utilizing waste products from the food production and processing industries. Crit. Rev. Food Sci. Nutr. 44, 57-62.

Salomone, R., Saija, G., Mondello, G., Giannetto, A., Fasulo, S., Savastano, D., 2017 Environmental impact of food waste bioconversion by insects: Application of Life Cycle Assessment to process using Hermetia illucens. J Clean Prod 140, 890905.

Sandrock, C., Leupi, S., Wohlfahrt, J., Leiber, F., Kreuzer, M., 2019. G x E interactions: Larval performance of distinct black soldier fly genotypes grown on different feed substrates, Book of Abstracts. Leibniz Institut für Agrartechnik und Bioökonomie eV, 49.

Sarpong, D., Oduro-Kwarteng, S., Gyasi, S.F., Buamah, R., Donkor, E., Awuah, E., Baah, M.K., 2019. Biodegradation by composting of municipal organic solid waste into organic fertilizer using the black soldier fly (Hermetia illucens) (Diptera: Stratiomyidae) larvae. Int J Recycl Org Waste Agricult 8, 45-54.

Schiavone, A., Cullere, M., De Marco, M., Meneguz, M., Biasato, I., Bergagna, S. Dezzutto, D., Gai, F., Dabbou, S., Gasco, L., Dalle, Z.A., 2016. Partial or total replacement of soybean oil by black soldier fly larvae (Hermetia illucens (L.)) fat in broiler diets: effect on growth performances, feed-choice, blood traits, carcass characteristics and meat quality. Ital. J. Anim. Sci. 16, 93-100.

Schiavone, A., Dabbou, S., De Marco, M., Cullere, M., Biasato, I., Biasibetti, E., Capucchio, M.T., Bergagna, S., Dezzutto, D., Meneguz, M., Gai, F., Dalle, Z.A. Gasco, L., 2018. Black soldier fly larva fat inclusion in finisher broiler chicken diet as an alternative fat source. Animal 12, 2032-2039.

Schiavone, A., Dabbou, S., Petracci, M., Zampiga, M., Sirri, F., Biasato, I., Gai, F., Gasco, L., 2019. Black soldier fly defatted meal as a dietary protein source for broile chickens: effects on carcass traits, breast meat quality and safety. Animal 13, 2397-2405.

Schiavone, A., De Marco, M., Martinez, S., Dabbou, S., Renna, M., Madrid, J. Hernandez, F., Rotolo, L., Costa, P., Gai, F., Gasco, L., 2017. Nutritional value of a partially defatted and a highly defatted black soldier fly larvae (Hermetia illucens (L.)) meal for broiler chickens: apparent nutrient digestibility, apparent metabolizable energy and apparent ileal amino acid digestibility. J Anim Sci Biotechnol 8, 51.

Sealey, W.M., Gaylord, T.G., Barrows, F.T., Tomberlin, J.K., McGuire, M.A., Ross, C., StHilaire, S., 2011. Sensory analysis of rainbow trout, Oncorhynchus mykiss, fed enriched black soldier fly prepupae, Hermetia illucens. J. World Aquacult. Soc. 42 34-45.

Secci, G., Bovera, F., Nizza, S., Baronti, N., Gasco, L., Conte, G., Serra, A., Bonelli, A. Parisi, G., 2018. Quality of eggs from Lohmann Brown Classic laying hens fed black soldier fly meal as substitute for soya bean. Animal 12, 2191-2197.

Secci, G., Bovera, F., Parisi, G., Moniello, G., 2020. Quality of Eggs and Albumen Technological Properties as Affected by Hermetia illucens Larvae Meal in Hens Diet and Hen Age. Animals 10.

Secci, G., Mancini, S., Iaconisi, V., Gasco, L., Basto, A., Parisi, G., 2019. Can the inclusion of black soldier fly (Hermetia illucens) in diet affect the flesh quality/ nutritional traits of rainbow trout (Oncorhynchus mykiss) after freezing and cooking? Int. J. Food Sci. Nutr. 70, 161-171.

Setti, L., Francia, E., Pulvirenti, A., Gigliano, S., Zaccardelli, M., Pane, C., Caradonia, F., Bortolini, S., Maistrello, L., Ronga, D., 2019. Use of black soldier fly (Hermetic illucens (L.), Diptera: Stratiomyidae) larvae processing residue in peat-based growing media. Waste Manag 95, 278-288.

Sharif, R., Mujtaba, M., Ur Rahman, M., Shalmani, A., Ahmad, H., Anwar, T., Tianchan, D., Wang, X., 2018. The Multifunctional Role of Chitosan in Horticultural Crops. A Review. Molecules 23, 872.

Sharp, R., 2013. A Review of the Applications of Chitin and Its Derivatives in Agriculture to Modify Plant-Microbial Interactions and Improve Crop Yields. Agronomy 3, 757-793.

Shelomi, M., Wu, M.K., Chen, S.M., Huang, J.J., Burke, C.G., 2020. Microbes Associated With Black Soldier Fly (Diptera: Stratiomiidae) Degradation of Food Waste. Environ Entomol 49, 405-411.

Sheppard, C., 1983. House fly and lesser fly control utilizing the black soldier fly in manure management systems for caged laying hens. Environ. Entomol. 12 1439-1442.

Sheppard, D.C., Newton, G.L., Thompson, S.A., Savage, S., 1994. A value added manure management system using the black soldier fly. Bioresour. Technol. 50, 275-279. 
Sheppard, D.C., Tomberlin, J.K., Joyce, J.A., Kiser, B.C., Sumner, S.M., 2002. Rearing methods for the black soldier fly (Diptera: Stratiomyidae). J. Med. Entomol. 39, 695-698.

Smetana, S., Palanisamy, M., Mathys, A., Heinz, V., 2016. Sustainability of insect use for feed and food: Life Cycle Assessment perspective. J. Clean Prod 137, 741751.

Smetana, S., Schmitt, E., Mathys, A., 2019. Sustainable use of Hermetia illucens insect biomass for feed and food: Attributional and consequential life cycle assessment. Resour Conserv Recy 144, 285-296.

Smets, R., Verbinnen, B., Van De Voorde, I., Aerts, G., Claes, J., Van Der Borght, M. 2020. Sequential Extraction and Characterisation of Lipids, Proteins, and Chitin from Black Soldier Fly (Hermetia illucens) Larvae, Prepupae, and Pupae. Waste Biomass Valor.

Soetemans, L., Uyttebroek, M., D’Hondt, E., Bastiaens, L., 2019. Use of organic acids to improve fractionation of the black soldier fly larvae juice into lipid- and protein-enriched fractions. Eur. Food Res. Technol. 245, 2257-2267.

Somroo, A.A., Ur Rehman, K., Zheng, L., Cai, M., Xiao, X., Hu, S., Mathys, A., Gold, M. $\mathrm{Yu}, \mathrm{Z}$., Zhang, J., 2019. Influence of Lactobacillus buchneri on soybean curd residue co-conversion by black soldier fly larvae (Hermetia illucens) for food and feedstock production. Waste Manag 86, 114-122.

Spranghers, T., Michiels, J., Vrancx, J., Ovyn, A., Eeckhout, M., De Clercq, P., De Smet, S., 2018. Gut antimicrobial effects and nutritional value of black soldier fly Hermetia illucens (L.)) prepupae for weaned piglets. Anim. Feed Sci. Technol. 235, 33-42.

Spranghers, T., Ottoboni, M., Klootwijk, C., Ovyn, A., Deboosere, S., De Meulenaer, B. Michiels, J., Eeckhout, M., De Clercq, P., De Smet, S., 2017. Nutritional composition of black soldier fly (Hermetia illucens) prepupae reared on different organic waste substrates. J Sci Food Agric 97, 2594-2600.

Stadtlander, T., Stamer, A., Buser, A., Wohlfahrt, J., Leiber, F., Sandrock, C., 2017. Hermetia illucens meal as fish meal replacement for rainbow trout on farm. J. Insects as Food Feed 3, 165-175.

Stenberg, O.K., Holen, E., Piemontese, L., Liland, N.S., Lock, E.J., Espe, M., Belghit, I. 2019. Effect of dietary replacement of fish meal with insect meal on in vitro bacterial and viral induced gene response in Atlantic salmon (Salmo salar) head kidney leukocytes. Fish Shellfish Immunol 91, 223-232.

St-Hilaire, S., Cranfill, K., McGuire, M.A., Mosley, E.E., Tomberlin, J.K., Newton, L. Sealey, W., Sheppard, C., Irving S., 2007a. Fish offal recycling by the black soldier fly produces a foodstuff high in omega-3 fatty acids. J. World Aquacult. Soc. $38,309-313$.

St-Hilaire, S., Sheppard, C., Tomberlin, J.K., Irving, S., Newton, L, McGuire, M.A. Mosley, E.E., Hardy, R.W., Sealey, W., 2007b. Fly prepupae as a feedstuff for rainbow trout Oncorhynchus mykiss. J. World Aquacult. Soc. 38, 59-67.

Su, C.H., Nguyen, H.C., Bui, T.L., Huang, D.L., 2019. Enzyme-assisted extraction of insect fat for biodiesel production. J. Clean. Prod. 223, 436-444.

Supriyatna, A., Manurung, R., Esyanthi, R.E., Putra, R.E., 2016. Growth of black soldier larvae fed on cassava peel wastes, An agriculture waste. J. Entomol. Zool. Stud. 4, 161-165.

Surendra, K.C., Kuehnle, A., 2019. Embracing AgTech for Food Security and Beyond. Ind Biotechnol 15, 323-324.

Surendra, K.C., Olivier, R., Tomberlin, J.K., Jha, R., Khanal, S.K., 2016. Bioconversion of organic wastes into biodiesel and animal feed via insect farming. Renew. Energy 98, 197-202.

Tabata, E., Kashimura, A., Wakita, S., Ohno, M., Sakaguchi, M., Sugahara, Y., Kino, Y. Matoska, V., Bauer, P.O., Oyama, F., 2017. Gastric and intestinal proteases resistance of chicken acidic chitinase nominates chitin-containing organisms for alternative whole edible diets for poultry. Sci. Rep. 7, 6662.

Terova, G., Rimoldi, S., Ascione, C., Gini, E., Ceccotti, C., Gasco, L., 2019. Rainbow trout (Oncorhynchus mykiss) gut microbiota is modulated by insect meal from Hermetia illucens prepupae in the diet. Rev. Fish Biol. Fish. 19, 465-486.

Tomberlin, J., Sheppard, D., Joyce, J., 2005. Black Soldier Fly (Diptera: Stratiomyidae) Colonization of Pig Carrion in South Georgia. J. Forensic Sci. 50, JFS20033912003392.

Tomberlin, J.K., Sheppard, D.C., Joyce, J.A., 2002. Selected Life-History Traits of Black Soldier Flies (Diptera: Stratiomyidae) Reared on Three Artificial Diets. Ann. Entomol. Soc. Am. 95, 379-386.

Tonk, M., Vilcinskas, A.J.C.t.i.m.c., 2017. The medical potential of antimicrobial peptides from insects. Curr. Top. Med. Chem. 17, 554-575.

Toriz-Roldan, A., Ruiz-Vega, J., García-Ulloa, M., Hernández-Llamas, A., FonsecaMadrigal, J., Rodríguez-González, H., 2019. Assessment of Dietary Supplementation Levels of Black Soldier Fly, Hemertia illucens, Pre-Pupae Mea for Juvenile Nile Tilapia, Oreochromis niloticus. Southwestern Entomologist 44, pp. 251-259.

Troschinetz, A.M., Mihelcic, J.R., 2009. Sustainable recycling of municipal solid waste in developing countries. Waste Manag 29, 915-923.

Truzzi, C., Giorgini, E., Annibaldi, A., Antonucci, M., Illuminati, S., Scarponi, G. Riolo, P. Isidoro, N., Conti, C., Zarantoniello, M., Cipriani, R., Olivotto, I., 2020. Fatty acids profile of black soldier fly (Hermetia illucens): Influence of feeding substrate based on coffee-waste silverskin enriched with microalgae. Anim. Feed Sci Technol, p. 259.

Tschirner, M., Simon, A., 2015. Influence of different growing substrates and processing on the nutrient composition of black soldier fly larvae destined for animal feed. J. Insects as Food Feed 1, 249-259.

Ushakova, N., Dontsov, A., Sakina, N., Bastrakov, A., Ostrovsky, M., 2019. Antioxidative Properties of Melanins and Ommochromes from Black Soldier Fly Hermetia illucens. Biomolecules 9. van der Fels-Klerx, H.J., Camenzuli, L., van der Lee, M.K., Oonincx, D.G., 2016. Uptake of Cadmium, Lead and Arsenic by Tenebrio molitor and Hermetia illucens from Contaminated Substrates. PLoS One 11, e0166186.

van Huis, A., 2019. Manure and flies: biodegradation and/or bioconversion? J. Insects as Food Feed 5, 55-58.

Varelas, V., 2019. Food Wastes as a Potential new Source for Edible Insect Mass Production for Food and Feed: A review. Fermentation 5.

Veldkamp, T., van Niekerk, T., 2019. Live black soldier fly larvae (Hermetia illucens) for turkey poults. J. Insects as Food Feed, 1-12.

Velten, S., Neumann, C., Dorper, A., Liebert, F., 2017. Response of piglets due to amino acid optimization of mixed diets with $75 \%$ replacement of soybeanmeal by partly defatted insect meal (H. illucens). INSECTA, Berlin, Germany, pp. 6364.

Verheyen, G.R., Ooms, T., Vogels, L., Vreysen, S., Bovy, A., Van, S.M., Meersman, F., 2018. Insects as an Alternative Source for the Production of Fats for Cosmetics. J Cosmet Sci 69, 187-202.

Vidanarachchi, J.K., Kurukulasuriya, M.S., Kim, S.K., 2010. Chitin, chitosan and their oligosaccharides in food industry. In: Kim, S.K. (Ed.), Chitin, Chitosan, Oligosaccharides and Their Derivatives: Biological Activities and Applications. CRC Press, New York, pp. 543-560.

Vogel, H., Muller, A., Heckel, D.G., Gutzeit, H., Vilcinskas, A., 2018. Nutritional immunology: Diversification and diet-dependent expression of antimicrobial peptides in the black soldier fly Hermetia illucens. Dev Comp Immunol 78, 141148.

Vongvichith, B., Morioka, S., Sugita, T., Phousavanh, N., Phetsanghanh, N., Chanthasone, P., Pommachan, P. Nakamura, S., 2020. Evaluation of the efficacy of aquaculture feeds for the climbing perch Anabas testudineus: replacement of fishmeal by black soldier fly Hermetia illucens prepupae. Fish. Sci. 86, 145-151.

Wallace, P.A., Nyameasem, J.K., Aboagye, G.A., Affedzie-Obresi, S., Nkegbe, K. Murray, F., Botchway, V., Karbo, N., Leschen, W., Maquart, P.O., Clottey, V., 2018. Effects of replacing fishmeal with black soldier fly larval meal in the diets of grower-finishing guinea fowls reared under tropical conditions. Trop Anim Health Prod 50, 1499-1507.

Wallace, P.A., Nyameasem, J.K., Adu-Aboagye, G.A., Affedzie-Obresi, S., Nkegbe, E.K., Karbo, N., Murray, F., Leschen, W., Maquart, P., 2017. Impact of black soldier fly larval meal on growth performance, apparent digestibility, haematological and blood chemistry indices of guinea fowl starter keets under tropical conditions. Trop Anim Health Prod 49, 1163-1169.

Wang, G., Peng, K., Hu, J., Yi, C., Chen, X., Wu, H., Huang, Y, 2019. Evaluation of defatted black soldier fly (Hermetia illucens (L.)) larvae meal as an alternative protein ingredient for juvenile Japanese seabass (Lateolabrax japonicus) diets. Aquaculture 507, 144-154.

Wang, H., Rehman, K.U., Liu, X., Yang, Q., Zheng, L., Li, W., Cai, M., Li, O., Zhang, J., Yu, Z., 2017. Insect biorefinery: a green approach for conversion of crop residues into biodiesel and protein. Biotechnol Biofuels 10, 304

Wang, S.Y., Wu, L., Li, B., Zhang, D., 2020. Reproductive Potential and Nutritional Composition of Hermetia illucens (Diptera: Stratiomyidae) Prepupae Reared on Different Organic Wastes. J Econ Entomol 113, 527-537.

Wang, Y.S., Shelomi, M., 2017. Review of Black Soldier Fly (Hermetia illucens) as Animal Feed and Human Food. Foods 6.

Wasko, A., Bulak, P., Polak-Berecka, M., Nowak, K., Polakowski, C., Bieganowski, A., 2016. The first report of the physicochemical structure of chitin isolated from Hermetia illucens. Int J Biol Macromol 92, 316-320.

Wilson, D.C., Rodic, L., Scheinberg, A., Velis, C.A., Alabaster, G., 2012. Comparative analysis of solid waste management in 20 cities. Waste Manag. Res. 30, $237-$ 254.

Wynants, E., Frooninckx, L., Crauwels, S., Verreth, C., De Smet, J., Sandrock, C., Wohlfahrt, J., Van Schelt, J., Depraetere, S., Lievens, B., Van Miert, S., Claes, J., Van Campenhout, L., 2019. Assessing the Microbiota of Black Soldier Fly Larvae (Hermetia illucens) Reared on Organic Waste Streams on Four Different Locations at Laboratory and Large Scale. Microb Ecol 77, 913-930.

Xiao, X., Jin, P., Zheng, L., Cai, M., Yu, Z., Yu, J., Zhang, J., 2018a. Effects of black soldier fly (Hermetia illucens) larvae meal protein as a fishmeal replacement on the growth and immune index of yellow catfish (Pelteobagrus fulvidraco). Aquacult. Res. 49, 1569-1577.

Xiao, X., Mazza, L., Yu, Y., Cai, M., Zheng, L., Tomberlin, J.K., Yu, J., van Huis, A., Yu, Z., Fasulo, S., Zhang, J., 2018b. Efficient co-conversion process of chicken manure into protein feed and organic fertilizer by Hermetia illucens (L.) (Diptera: Stratiomyidae) larvae and functional bacteria. J Environ Manage 217, 668-676.

Yazan, D.M., Fraccascia, L., Mes, M., Zijm, H., 2018. Cooperation in manure-based biogas production networks: An agent-based modeling approach. J Applied energy 212, 820-833.

Yi, H.Y., Chowdhury, M., Huang, Y.D., Yu, X.Q., 2014. Insect antimicrobial peptides and their applications. Appl. Microbiol. Biotechnol. 98, 5807-5822.

Yin, J., Wang, K., Yang, Y., Shen, D., Wang, M., Mo, H., 2014. Improving production of volatile fatty acids from food waste fermentation by hydrothermal pretreatment. Bioresour. Technol. 171, 323-329.

Yu, G., Cheng, P., Chen, Y., Li, Y., Yang, Z., Chen, Y., Tomberlin, J.K., 2011. Inoculating poultry manure with companion bacteria influences growth and development of black soldier fly (Diptera: Stratiomyidae) larvae. Environ Entomol 40, 30-35.

Yu, M., Li, Z., Chen, W., Rong, T., Wang, G., Li, J., Ma, X., 2019a. Use of Hermetia illucens larvae as a dietary protein source: Effects on growth performance, carcass traits, and meat quality in finishing pigs. Meat Sci 158, 107837 
Yu, M., Li, Z., Chen, W., Rong, T., Wang, G., Ma, X., 2019b. Hermetia illucens larvae as a potential dietary protein source altered the microbiota and modulated mucosal immune status in the colon of finishing pigs. J Anim Sci Biotechnol 10, 50.

Zarantoniello, M., Randazzo, B., Truzzi, C., Giorgini, E., Marcellucci, C., VargasAbundez, J.A., Zimbelli, A., Annibaldi, A., Parisi, G., Tulli, F., Riolo, P., Olivotto, I., 2019. A six-months study on Black Soldier Fly (Hermetia illucens) based diets in zebrafish. Sci. Rep. 9, 8598.

Zhan, S., Fang, G., Cai, M., Kou, Z., Xu, J., Cao, Y., Bai, L., Zhang, Y., Jiang, Y., Luo, X., Xu, J., Xu, X., Zheng, L., Yu, Z., Yang, H., Zhang, Z., Wang, S., Tomberlin, J.K., Zhang, J. Huang, Y., 2020. Genomic landscape and genetic manipulation of the black soldier fly Hermetia illucens, a natural waste recycler. Cell Res. 30, 50-60.

Zhao, W., Lu, L., Tang, Y., 2010. Research and application progress of insect antimicrobial peptides on food industry. Int. J Food Eng 6.

Zheng, L., Crippen, T.L., Singh, B., Tarone, A.M., Dowd, S., Yu, Z., Wood, T.K., Tomberlin, J.K., 2013. A survey of bacterial diversity from successive life stages of black soldier fly (Diptera: Stratiomyidae) by using 16S rDNA pyrosequencing. J Med Entomol 50, 647-658.

Zheng, L., Hou, Y., Li, W., Yang, S., Li, Q., Yu, Z., 2012a. Biodiesel production from rice straw and restaurant waste employing black soldier fly assisted by microbes. Energy 47, 225-229.

Zhou, F., Tomberlin, J.K., Zheng, L., Yu, Z., Zhang, J., 2013a. Developmental and Waste Reduction Plasticity of Three Black Soldier Fly Strains (Diptera: Stratiomyidae) Raised on Different Livestock Manures. J. Med. Entomol. 50, 1224-1230.

Zhou, Z., Karlsen, Ø., He, S., Olsen, R.E., Yao, B., Ringø, E., 2013. The effect of dietary chitin on the autochthonous gut bacteria of Atlantic cod (Gadus morhua (L.)). Aquac. Res. 44, 1889-1900.
Zhou, J.S., Liu, S.S., Ji, H., Yu, H.B., 2018. Effect of replacing dietary fish meal with black soldier fly larvae meal on growth and fatty acid composition of Jian carp (Cyprinus carpio var. Jian). Aquac Nutr 24, 424-433.

Zheng, L., Li, Q., Zhang, J., Yu, Z., 2012. Double the biodiesel yield: Rearing black soldier fly larvae, Hermetia illucens, on solid residual fraction of restaurant waste after grease extraction for biodiesel production. Renew. Energy 41, 75-79.

Zhu, Z., Rehman, K.U., Yu, Y., Liu, X., Wang, H., Tomberlin, J.K., Sze, S.H., Cai, M., Zhang, J., Yu, Z., Zheng, J., Zheng, L., 2019. De novo transcriptome sequencing and analysis revealed the molecular basis of rapid fat accumulation by black soldier fly (Hermetia illucens (L.)) for development of insectival biodiesel Biotechnol Biofuels 12, 194.

Zotte, A.D., Singh, Y., Michiels, J., Cullere, M., 2019. Black Soldier Fly (Hermetic illucens) as Dietary Source for Laying Quails: Live Performance, and Egg PhysicoChemical Quality. Sensory Profile and Storage Stability, Animals (Basel), p. 9.

Zurbrügg, C., Dortmans, B., Fadhila, A., Verstappen, B., Diener, S., 2018. From pilot to full scale operation of a waste-to-protein treatment facility. Detritus 1, 18.

\section{Further reading}

Singh, A., Kumari, K., 2019. An inclusive approach for organic waste treatment and valorisation using Black Soldier Fly larvae: A review. J Environ Manage 251 109569. 